\title{
Improved methodologies for the preparation of highly
}

\section{substituted pyridines}

\author{
Yolanda Fernández Sainz, Steven A. Raw ${ }^{\#}$ and Richard J. K. Taylor* \\ Department of Chemistry, University of York, Heslington, York YO10 5DD, U.K. \\ E-mail: rjkt1@york.ac.uk \\ ${ }^{\#}$ Current address: Process Research and Development, AstraZeneca, Macclesfield, SK10 2NA, UK.
}

\section{Table of Contents}

General Experimental Methods $\quad$ S2

$\begin{array}{ll}\text { Synthesis of highly substituted pyridines } 5,8 \text { and } 9 & \text { S3 }\end{array}$

$\begin{array}{ll}\text { General procedure }(\mathrm{A}) \text { : microwave-promoted solvent-free procedure } & \mathbf{S 3}\end{array}$

General procedure (B): tethered imine-enamine (TIE) methodology $\quad$ S3

Experimental procedures and spectra data $\left({ }^{1} \mathrm{H}\right.$ NMR, ${ }^{13} \mathrm{C}$ NMR, IR, LRMS, HRMS)

3-Phenyl-1-pyridin-2-yl-6,7-dihydro-5H-[2]pyrindine (5a) $\quad$ S4

3-Phenyl-1-pyridin-2-yl-4-pyrrolidin-1-yl-6,7-dihydro-5H-[2]pyrindine $\quad$ S4

3,4-Difuran-2-yl-1-pyridin-2-yl-6,7-dihydro-5H-[2]pyrindine (5b) S6

3,4-Difuran-2-yl-1-pyridin-2-yl-5,6,7,8-tetrahydro-isoquinoline (5c) $\quad$ S7

3-Phenyl-6,7-dihydro-5H-[2]pyrindine-1-carboxylic acid ethyl ester (5d) $\quad \mathbf{S 8}$

3,4-Dimethyl-6,7-dihydro-5H-[2]pyrindine-1-carboxylic acid ethyl ester (5e) S9

1-Pyridin-2-yl-6,7-dihydro-5H-[2]pyrindine (5f) $\quad$ S9

3-Phenyl-1-pyridin-2-yl-5,6,7,8-tetrahydro-isoquinoline (5g) $\quad$ S10

1-Pyridin-2-yl-5,6,7,8-tetrahydro-isoquinoline (5h) $\quad$ S11

3-Phenyl-1-pyridin-2-yl-6,7,8,9-tetrahydro-5H-cyclohepta[c]pyridine (5i) $\quad$ S12

3,4-Di-furan-2-yl-1-pyridin-2-yl-6,7,8,9-tetrahydro-5H-cyclohepta[c]pyridine (5j) $\quad$ S13

1-Pyridin-2-yl-6,7,8,9-tetrahydro-5H-cyclohepta[c]pyridine (5k) $\quad \mathbf{S 1 4}$

3-Phenyl-1-pyridin-2-yl-5,6,7,8,9,10-hexahydro-cycloocta[c]pyridine (5l) $\quad$ S15 
3-Phenyl-1-pyridin-2-yl-5,6,7,8,9,10,11,12,13,14-decahydro-cyclododeca[c]pyridine (5m) $\quad$ S16

3-Phenyl-5,6,7,8-tetrahydro-isoquinoline-1-carboxylic acid ethyl ester (5n) $\quad$ S17

2-Phenyl-4-pyridin-2-yl-9H-3-aza-fluorene (8a) $\quad$ S18

3-Phenyl-1-pyridin-2-yl-5,6-dihydro-benzo[h]isoquinoline (8b) $\quad$ S19

2-Phenyl-4-pyridin-2-yl-5,6-dihydro-benzo[f]isoquinoline (9b) S19

3-Phenyl-1-pyridin-2-yl-benzo[4,5]furo[3,2-c]pyridine (8c) $\quad$ S20

3,6-Diphenyl-[2,2] bipyridinyl (8d) $\quad$ S21

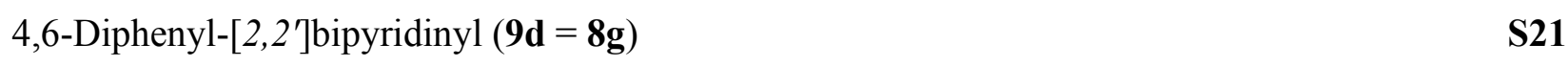

3-Ethyl-4-methyl-6-phenyl-[2,2] bipyridinyl (8e) $\quad$ S23

4-Ethyl-3-methyl-6-phenyl-[2,2] bipyridinyl (9e) $\quad$ S23

6-Phenyl-4-propyl-[2,2]bipyridinyl (8f) $\quad$ S24

5,6-Difuran-2-yl-3-propyl-[2,2] bipyridinyl (9h) $\quad$ S25

5,6-Difuran-2-yl-3-phenyl-[2,2]bipyridinyl (9i) $\quad$ S26

4-Propyl-[2,2]bipyridinyl (9j) $\quad$ S27

${ }^{1}$ H NMR and ${ }^{13}$ NMR Spectra $\quad$ S28

\section{General Experimental Methods}

${ }^{1} \mathrm{H}$ NMR and ${ }^{13} \mathrm{C}$ NMR spectra in $\mathrm{CDCl}_{3}$ solution were recorded on 270 and $400 \mathrm{MHz}$ spectrometers (with TMS for ${ }^{1} \mathrm{H}$ and chloroform-d for ${ }^{13} \mathrm{C}$ as the internal reference); NOESY experiments were carried out on a $500 \mathrm{MHz}$ spectrometer in order to establish atom connectivities and ${ }^{1} \mathrm{H}$ NMR peak assignments for compounds $8 \mathbf{a}, 8 \mathbf{b}, 8 \mathbf{c}, 8 \mathrm{~d}, \mathbf{8 e}, \mathbf{8 f}, \mathbf{9 b}, \mathbf{9 d}, 9 \mathbf{e}, 9 \mathbf{h}, 9 \mathbf{i}$ and $\mathbf{9 j}$. Infrared spectra were recorded using $\mathrm{NaCl}$ plates on an FT-IR spectrophotometer. Low- and high-resolution mass spectra were obtained for all novel compounds. Melting points were determined on a digital melting point apparatus and are uncorrected. TLC was performed on aluminium-backed plates coated with silica gel 60 with $\mathrm{F}_{254}$ indicator and compounds were visualized by UV light $(254 \mathrm{~nm})$ or iodine. Flash column chromatography was performed on Matrex silica gel 60 (220-440 mesh ASTM) and $n$-hexane/EtOAc solvent systems. Microwave reactions were performed using a focused microwave unit. The temperature was measured by infrared detection with continuous feedback temperature control, and maintained at a constant value by power modulation $(0-300 \mathrm{~W})$. The reactions were performed in glass 
vessels (10 mL capacity) sealed with a septum. The pressure was controlled by a load cell connected to the vessel which senses changes in the external deflection of the septa on top of the sealed pressure vessel. All commercial reagents were used directly unless otherwise noted.

\section{Synthesis of highly substituted pyridines 5,8 and 9}

\section{General procedure A: Preparation of highly substituted pyridines by microwave-promoted solvent-free procedure.}

1,2,4-Triazine 1 ( $0.1 \mathrm{mmol})$, pyrrolidine ( 1 equiv. unless otherwise noted) and ketone ( 1 equiv. unless otherwise noted) were mixed together into a $10 \mathrm{~mL}$ glass vessel sealed with a septum and irradiated for the time and the temperature given in Tables 1,2 and 3, maintaining this temperature by power modulation between 150 and $200 \mathrm{~W}$. The vessel was cooled down and diluted with dichloromethane (ca. $1 \mathrm{ml}$ ) followed by concentration in vacuo and purification by flash chromatography on silica gel.

\section{General procedure B: Preparation of highly substituted pyridines by tethered imine-enamine} (TIE) methodology.

To a solution of 1,2,4-triazine $1(0.1 \mathrm{mmol})$ in toluene $(1.0 \mathrm{ml})$ was added powdered $4 \mathrm{~A}$ molecular sieves $(0.100 \mathrm{~g})$, ketone $(0.60 \mathrm{mmol})$ and $N$-methylethylenediamine $(0.30 \mathrm{mmol})$ and the mixture heated at $120^{\circ} \mathrm{C}$ for the time given in Table 6 . The reaction mixture was then cooled, filtered through a cotton wool plug, concentrated in vacuo and purified by column chromatography on silica gel. 
<smiles>c1ccc(-c2cc3c(c(-c4ccccn4)n2)CCC3)cc1</smiles><smiles>c1ccc(-c2nc(-c3ccccn3)c3c(c2N2CCCC2)CCC3)cc1</smiles>

\section{3-Phenyl-1-pyridin-2-yl-4-pyrrolidin-1-yl-6,7-dihydro-5H-}

\section{[2]pyrindine}

\section{Procedure A:}

A mixture of 5-phenyl-3-pyridin-2-yl-[1,2,4]triazine $(0.023 \mathrm{~g}, 0.1 \mathrm{mmol})$, pyrrolidine $(0.009 \mathrm{ml}, 0.1$ mmol) and cyclopentanone $(0.009 \mathrm{ml}, 0.1 \mathrm{mmol})$ was irradiated at $120^{\circ} \mathrm{C}$ for $20 \mathrm{~min}$. An analysis of the crude reaction by ${ }^{1} \mathrm{H}$ NMR spectroscopy showed a mixture of 3-phenyl-1-pyridin-2-yl-6,7-dihydro-5H[2]pyrindine $\mathbf{5 a}$ and 3-phenyl-1-pyridin-2-yl-4-pyrrolidin-1-yl-6,7-dihydro-5 $H$-[2]pyrindine in a proportion of 10:1 respectively. The mixture was separated by flash chromatography on silica gel (petroleum ether:ethyl acetate, 5:1) to give 5a $(0.024 \mathrm{~g}, 88 \%)$ and the corresponding 4-functionalized derivative $(0.003 \mathrm{~g}, 9 \%)$.

\section{Procedure B:}

A mixture of 5-phenyl-3-pyridin-2-yl-[1,2,4]triazine $(0.023 \mathrm{~g}, 0.1 \mathrm{mmol}), N$-methylethylenediamine $(0.026 \mathrm{ml}, 0.30 \mathrm{mmol})$, cyclopentanone $(0.054 \mathrm{ml}, 0.6 \mathrm{mmol})$ and powdered $4 \mathrm{~A}$ molecular sieves $(0.100 \mathrm{~g})$ in toluene $(1.0 \mathrm{ml})$ was heated at $120^{\circ} \mathrm{C}$ for $22 \mathrm{~h}$. Purification by flash chromatography on silica gel (petroleum ether:ethyl acetate, 5:1) gave the title compound 5a (0.021 g, 74\%). 
For 5a. White solid: $\mathrm{mp} 110-111^{\circ} \mathrm{C}$ (hexane- $\mathrm{CHCl}_{3}$ ); $v_{\max }$ (film) 2923, 2853, 1583, 1427, 1372, 863 $\mathrm{cm}^{-1} ; \delta_{\mathrm{H}}\left(400 \mathrm{MHz}, \mathrm{CDCl}_{3}\right) 8.70(1 \mathrm{H}, \mathrm{ddd}, J=4.8,2.0,0.8 \mathrm{~Hz}), 8.46(1 \mathrm{H}, \mathrm{ddd}, J=7.6,1.2,0.8 \mathrm{~Hz})$, 8.13-8.09 (2 H, m), $7.83(1 \mathrm{H}, \mathrm{td}, J=7.6,2.0 \mathrm{~Hz}), 7.68(1 \mathrm{H}, \mathrm{s}), 7.51-7.45(2 \mathrm{H}, \mathrm{m}), 7.43-7.38(1 \mathrm{H}$, m), $7.28(1 \mathrm{H}, \mathrm{ddd}, J=7.6,4.8,1.2 \mathrm{~Hz}), 3.47(2 \mathrm{H}, \mathrm{t}, J=7.2 \mathrm{~Hz}), 3.02(2 \mathrm{H}, \mathrm{t}, J=7.2 \mathrm{~Hz}), 2.15(2 \mathrm{H}$, quint, $J=7.2 \mathrm{~Hz}) ; \delta_{\mathrm{C}}\left(100 \mathrm{MHz}, \mathrm{CDCl}_{3}\right) 158.6(\mathrm{C}), 156.8(\mathrm{C}), 154.5(\mathrm{C}), 151.3(\mathrm{C}), 148.4(\mathrm{CH}), 139.9$ (C), $137.9(\mathrm{C}), 136.3(\mathrm{CH}), 128.6(2 \times \mathrm{CH}), 128.5(\mathrm{CH}), 126.9(2 \times \mathrm{CH}), 123.1(\mathrm{CH}), 122.7(\mathrm{CH})$, $116.5(\mathrm{CH}), 32.9\left(\mathrm{CH}_{2}\right), 32.8\left(\mathrm{CH}_{2}\right), 25.1\left(\mathrm{CH}_{2}\right) ; \mathrm{m} / \mathrm{z}(\mathrm{EI}) 272\left(80 \%, \mathrm{M}^{+}\right), 271\left(100 \%, \mathrm{M}^{+}-1\right)[\mathrm{HRMS}$ (EI) calcd. for $\mathrm{C}_{19} \mathrm{H}_{15} \mathrm{~N}_{2}\left(\mathrm{M}^{+}-1\right)$ 271.1235. Found 271.1238 (-3.3 ppm error)].

For 3-phenyl-1-pyridin-2-yl-4-pyrrolidin-1-yl-6,7-dihydro-5H-[2]pyrindine. Yellow oil: $\boldsymbol{R}_{\mathrm{f}} \mathbf{0 . 2 0}$ (petroleum ether:ethyl acetate, 5:1); $\delta_{\mathrm{H}}\left(400 \mathrm{MHz}, \mathrm{CDCl}_{3}\right) 8.64(1 \mathrm{H}$, ddd, $J=4.8,2.0,0.8 \mathrm{~Hz}, 1-(2-$ pyridyl), $8.23(1 \mathrm{H}, \mathrm{ddd}, J=7.6,1.2,0.8 \mathrm{~Hz}), 7.76-7.70(3 \mathrm{H}, \mathrm{m}), 7.43-7.38(2 \mathrm{H}, \mathrm{m}), 7.34-7.29(1 \mathrm{H}$, m), $7.18(1 \mathrm{H}, \mathrm{ddd}, J=7.6,4.8,1.2 \mathrm{~Hz}), 3.40(2 \mathrm{H}, \mathrm{t}, J=7.6 \mathrm{~Hz}), 3.11-3.06(4 \mathrm{H}, \mathrm{m}), 3.02(2 \mathrm{H}, \mathrm{t}, J=$ $7.6 \mathrm{~Hz}), 2.12(2 \mathrm{H}$, quint, $J=7.6 \mathrm{~Hz}), 1.81-1.76(4 \mathrm{H}, \mathrm{m}) ; \delta_{\mathrm{C}}\left(100 \mathrm{MHz}, \mathrm{CDCl}_{3}\right) 158.6(\mathrm{C}), 151.1(\mathrm{C})$, $149.1(\mathrm{C}), 148.2(\mathrm{CH}), 145.3(\mathrm{C}), 141.9(\mathrm{C}), 139.9(\mathrm{C}), 139.5(\mathrm{C}), 136.1(\mathrm{CH}), 128.8(2 \times \mathrm{CH}), 127.9$ $(2 \times \mathrm{CH}), 127.2(\mathrm{CH}), 122.9(\mathrm{CH}), 121.8(\mathrm{CH}), 50.3\left(2 \times \mathrm{CH}_{2}\right), 32.9\left(\mathrm{CH}_{2}\right), 32.7\left(\mathrm{CH}_{2}\right), 25.8\left(2 \times \mathrm{CH}_{2}\right)$, $25.7\left(\mathrm{CH}_{2}\right) ; \mathrm{m} / \mathrm{z}(\mathrm{CI}) 342\left(\mathrm{MH}^{+}, 100 \%\right)$ [HRMS (CI): calcd. for $\mathrm{C}_{23} \mathrm{H}_{24} \mathrm{~N}_{3}, 342.1970$. Found: $\mathrm{MH}^{+}$, $342.1974(-1.0$ ppm error)]. 


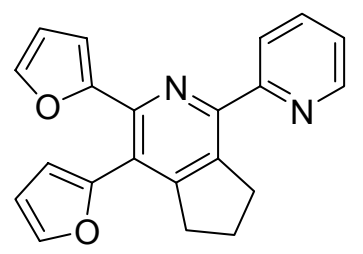

3,4-Difuran-2-yl-1-pyridin-2-yl-6,7-dihydro-5 $\mathrm{H}$ -

[2]pyrindine (5b)

\section{Procedure A:}

A mixture of 5,6-difuran-2-yl-3-pyridin-2-yl-[1,2,4]triazine $(0.029 \mathrm{~g}, 0.1 \mathrm{mmol})$, pyrrolidine $(0.009 \mathrm{ml}$, $0.1 \mathrm{mmol})$ and cyclopentanone $(0.009 \mathrm{ml}, 0.1 \mathrm{mmol})$ was irradiated at $120^{\circ} \mathrm{C}$ for $20 \mathrm{~min}$. Purification by flash chromatography on silica gel (petroleum ether:ethyl acetate, 4:1) gave the title compound $\mathbf{5 b}$ $(0.030 \mathrm{~g}, 92 \%)$.

\section{Procedure B:}

A mixture of 5,6-difuran-2-yl-3-pyridin-2-yl-[1,2,4]triazine $\quad(0.029 \mathrm{~g}, \quad 0.1 \mathrm{mmol}), \quad N-$ methylethylenediamine $(0.026 \mathrm{ml}, 0.30 \mathrm{mmol})$, cyclopentanone $(0.054 \mathrm{ml}, 0.6 \mathrm{mmol})$ and powdered 4A molecular sieves $(0.100 \mathrm{~g})$ in toluene $(1.0 \mathrm{ml})$ was heated at $120^{\circ} \mathrm{C}$ for $22 \mathrm{~h}$. Purification by flash chromatography on silica gel (petroleum ether:ethyl acetate, 4:1) gave the title compound $\mathbf{5 b}(0.029 \mathrm{~g}, 88 \%)$.

White solid: $\mathrm{mp} 108-109^{\circ} \mathrm{C}$ (hexane- $\mathrm{CHCl}_{3}$ ); $v_{\max }$ (film) 2923, 1547, 1471, 1392, 1260, 1012, $739 \mathrm{~cm}^{-}$ ${ }^{1} ; \delta_{\mathrm{H}}\left(400 \mathrm{MHz}, \mathrm{CDCl}_{3}\right) 8.70(1 \mathrm{H}, \mathrm{ddd}, J=4.8,2.0,0.8 \mathrm{~Hz}), 8.36(1 \mathrm{H}, \mathrm{ddd}, J=8.0,1.2,0.8 \mathrm{~Hz}), 7.82$ $(1 \mathrm{H}, \mathrm{td}, J=8.0,2.0 \mathrm{~Hz}), 7.53(1 \mathrm{H}, \mathrm{dd}, J=2.0,0.8 \mathrm{~Hz}), 7.45(1 \mathrm{H}, \mathrm{dd}, J=2.0,0.8 \mathrm{~Hz}), 7.28(1 \mathrm{H}$, ddd, $J=8.0,4.8,1.2 \mathrm{~Hz}), 6.54(1 \mathrm{H}, \mathrm{dd}, J=3.6,2.0 \mathrm{~Hz}), 6.40(1 \mathrm{H}, \mathrm{dd}, J=3.6,2.0 \mathrm{~Hz}), 6.35(1 \mathrm{H}, \mathrm{dd}, J=$ 3.6, $0.8 \mathrm{~Hz}), 6.23(1 \mathrm{H}, \mathrm{dd}, J=3.6,0.8 \mathrm{~Hz}), 3.49(2 \mathrm{H}, \mathrm{t}, J=7.6 \mathrm{~Hz}), 2.94(2 \mathrm{H}, \mathrm{t}, J=7.6 \mathrm{~Hz}), 2.17-$ $2.04(2 \mathrm{H}$, quint, $J=7.6 \mathrm{~Hz}) ; \delta_{\mathrm{C}}\left(100 \mathrm{MHz}, \mathrm{CDCl}_{3}\right) 157.9(\mathrm{C}), 157.1(\mathrm{C}), 152.9(\mathrm{C}), 151.1(\mathrm{C}), 149.9$ (C), $148.5(\mathrm{CH}), 145.6(\mathrm{C}), 142.7(\mathrm{CH}), 142.1(\mathrm{CH}), 137.8(\mathrm{C}), 136.4(\mathrm{CH}), 123.4(\mathrm{CH}), 122.9(\mathrm{CH})$, $120.7(\mathrm{C}), 111.3(\mathrm{CH}), 111.1(\mathrm{CH}), 110.3(\mathrm{CH}), 109.7(\mathrm{CH}), 33.4\left(\mathrm{CH}_{2}\right), 32.6\left(\mathrm{CH}_{2}\right), 24.9\left(\mathrm{CH}_{2}\right) ; \mathrm{m} / \mathrm{z}$ (EI) $328\left(85 \%, \mathrm{M}^{+}\right), 327\left(100 \%, \mathrm{M}^{+}-1\right)$ [HRMS (EI) calcd. for $\mathrm{C}_{21} \mathrm{H}_{16} \mathrm{~N}_{2} \mathrm{O}_{2}$ 328.1211. Found 328.1208 (1.0 ppm error)]. 


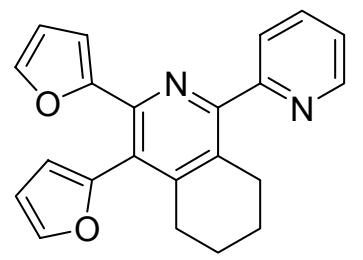

\section{3,4-Difuran-2-yl-1-pyridin-2-yl-5,6,7,8-tetrahydro- isoquinoline (5c)}

\section{Procedure A:}

A mixture of 5,6-difuran-2-yl-3-pyridin-2-yl-[1,2,4]triazine $(0.029 \mathrm{~g}, 0.1 \mathrm{mmol})$, pyrrolidine $(0.009 \mathrm{ml}$, $0.1 \mathrm{mmol})$ and cyclohexanone $(0.010 \mathrm{ml}, 0.1 \mathrm{mmol})$ was irradiated at $120^{\circ} \mathrm{C}$ for $20 \mathrm{~min}$. Purification by flash chromatography on silica gel (petroleum ether:ethyl acetate, 4:1) gave the title compound 5c $(0.022 \mathrm{~g}, 64 \%)$.

\section{Procedure B:}

A mixture of 5,6-difuran-2-yl-3-pyridin-2-yl-[1,2,4]triazine $(0.029 \mathrm{~g}, \quad 0.1 \mathrm{mmol}), \quad N-$ methylethylenediamine $(0.026 \mathrm{ml}, 0.30 \mathrm{mmol})$, cyclohexanone $(0.060 \mathrm{ml}, 0.6 \mathrm{mmol})$ and powdered $4 \mathrm{~A}$ molecular sieves $(0.100 \mathrm{~g})$ in toluene $(1.0 \mathrm{ml})$ was heated at $120^{\circ} \mathrm{C}$ for $21 \mathrm{~h}$. Purification by flash chromatography on silica gel (petroleum ether:ethyl acetate, $4: 1)$ gave the title compound $\mathbf{5 c}(0.028 \mathrm{~g}, 82 \%)$.

White solid: $\mathrm{mp} 141-142^{\circ} \mathrm{C}$ (hexane- $\mathrm{CHCl}_{3}$ ); $v_{\max }$ (film) 2932, 1588, 1536, 1409, 1151, 1016, 801, 743 $\mathrm{cm}^{-1} ; \delta_{\mathrm{H}}\left(400 \mathrm{MHz}, \mathrm{CDCl}_{3}\right) 8.67(1 \mathrm{H}, \mathrm{ddd}, J=4.8,1.2,0.8 \mathrm{~Hz}), 7.85-7.79(2 \mathrm{H}, \mathrm{m}), 7.60(1 \mathrm{H}, \mathrm{dd}, J=$ 2.0, $0.8 \mathrm{~Hz}), 7.42(1 \mathrm{H}, \mathrm{dd}, J=2.0,0.8 \mathrm{~Hz}), 7.30(1 \mathrm{H}, \mathrm{ddd}, J=6.4,4.8,1.6 \mathrm{~Hz}), 6.56(1 \mathrm{H}, \mathrm{dd}, J=3.2$, $2.0 \mathrm{~Hz}), 6.32-6.29(2 \mathrm{H}, \mathrm{m}) 5.79(1 \mathrm{H}, \mathrm{dd}, J=3.2,0.8 \mathrm{~Hz}), 2.91(2 \mathrm{H}, \mathrm{t}, J=6.4 \mathrm{~Hz}), 2.62(2 \mathrm{H}, \mathrm{t}, J=$ $6.0 \mathrm{~Hz}), 1.80-1.68(4 \mathrm{H}, \mathrm{m}) ; \delta_{\mathrm{C}}\left(100 \mathrm{MHz}, \mathrm{CDCl}_{3}\right) 158.9(\mathrm{C}), 156.8(\mathrm{C}), 152.0(\mathrm{C}), 149.5(\mathrm{C}), 149.4$ (C), $148.3(\mathrm{CH}), 145.7(\mathrm{C}), 142.9(\mathrm{CH}), 142.3(\mathrm{CH}), 136.7(\mathrm{CH}), 130.3(\mathrm{C}), 124.7(\mathrm{CH}), 123.0(\mathrm{C})$, $122.8(\mathrm{CH}), 111.2(\mathrm{CH}), 111.2(\mathrm{CH}), 110.3(\mathrm{CH}), 109.7(\mathrm{CH}), 27.8\left(\mathrm{CH}_{2}\right), 27.2\left(\mathrm{CH}_{2}\right), 22.4\left(\mathrm{CH}_{2}\right)$, $22.0\left(\mathrm{CH}_{2}\right) ; \mathrm{m} / \mathrm{z}(\mathrm{EI}) 342\left(100 \%, \mathrm{M}^{+}\right)$[HRMS (EI) calcd. for $\mathrm{C}_{22} \mathrm{H}_{18} \mathrm{~N}_{2} \mathrm{O}_{2}$ 342.1368. Found 342.1362 (1.8 ppm error)]. 


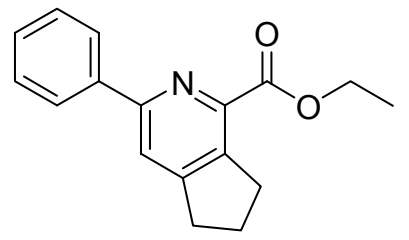

3-Phenyl-6,7-dihydro-5 $H$-[2]pyrindine-1-carboxylic acid ethyl ester (5d)

\section{Procedure A:}

A mixture of 5-phenyl-[1,2,4]triazine-3-carboxylic acid ethyl ester $(0.023 \mathrm{~g}, 0.1 \mathrm{mmol})$, pyrrolidine $(0.009 \mathrm{ml}, 0.1 \mathrm{mmol})$ and cyclopentanone $(0.009 \mathrm{ml}, 0.1 \mathrm{mmol})$ was irradiated at $120^{\circ} \mathrm{C}$ for $30 \mathrm{~min}$. Purification by flash chromatography on silica gel (petroleum ether:ethyl acetate, 5:1) gave the title compound 5d (0.018 g, $67 \%)$.

\section{Procedure B:}

A mixture of 5-phenyl-[1,2,4]triazine-3-carboxylic acid ethyl ester $(0.023 \mathrm{~g}, 0.1 \mathrm{mmol}), \mathrm{N}$ methylethylenediamine $(0.026 \mathrm{ml}, 0.30 \mathrm{mmol})$, cyclopentanone $(0.054 \mathrm{ml}, 0.6 \mathrm{mmol})$ and powdered 4A molecular sieves $(0.100 \mathrm{~g})$ in toluene $(1.0 \mathrm{ml})$ was heated at $120^{\circ} \mathrm{C}$ for $6 \mathrm{~h}$. Purification by flash chromatography on silica gel (petroleum ether:ethyl acetate, 4:1) gave the title compound 5d (0.009 g, $33 \%)$.

Colorless oil $R_{\mathrm{f}} 0.50$ (petroleum ether:ethyl acetate 1:1); $v_{\max }$ (film) 2974, 2928, 1714, 1598, 1448, 1369, 1224, 1137, 1031, $780 \mathrm{~cm}^{-1} ; \delta_{\mathrm{H}}\left(400 \mathrm{MHz}, \mathrm{CDCl}_{3}\right)$ 8.05-8.01 (2 H, m), 7.76 (1 H, s), 7.49-7.44 (2 H, m), 7.42-7.38 (1 H, m), $4.47(2 \mathrm{H}, \mathrm{q}, J=7.2 \mathrm{~Hz}), 3.30(2 \mathrm{H}, \mathrm{t}, J=7.6 \mathrm{~Hz}), 3.02(2 \mathrm{H}, \mathrm{t}, J=7.6 \mathrm{~Hz})$, $2.16(2 \mathrm{H}$, quint, $J=7.6 \mathrm{~Hz}), 1.46(3 \mathrm{H}, \mathrm{t}, J=7.2 \mathrm{~Hz}) ; \delta_{\mathrm{C}}\left(100 \mathrm{MHz}, \mathrm{CDCl}_{3}\right) 166.1(\mathrm{CO}), 157.3(\mathrm{C})$, $155.4(\mathrm{C}), 143.5(\mathrm{C}), 141.5(\mathrm{C}), 139.0(\mathrm{C}), 128.9(\mathrm{CH}), 128.7(2 \times \mathrm{CH}), 127.1(2 \times \mathrm{CH}), 119.7(\mathrm{CH})$, $61.4\left(\mathrm{CH}_{2}\right), 32.6\left(\mathrm{CH}_{2}\right), 32.3\left(\mathrm{CH}_{2}\right), 24.6\left(\mathrm{CH}_{2}\right), 14.3\left(\mathrm{CH}_{3}\right) ; \mathrm{m} / \mathrm{z}(\mathrm{EI}) 267\left(41 \%, \mathrm{M}^{+}\right), 195\left(100 \%, \mathrm{M}^{+}-\right.$ 72) [HRMS (EI) calcd. for $\mathrm{C}_{17} \mathrm{H}_{17} \mathrm{NO}_{2} 267.1259$. Found 267.1263 (-1.8 ppm error)]. 


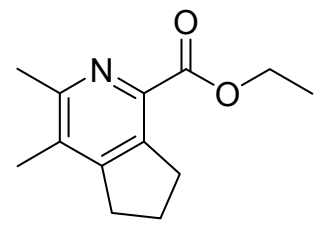

\section{3,4-Dimethyl-6,7-dihydro-5H-[2]pyrindine-1-carboxylic} acid ethyl ester (5e)

\section{Procedure A:}

A mixture of 5,6-dimethyl-[1,2,4]triazine-3-carboxylic acid ethyl ester (0.018 g, $0.1 \mathrm{mmol})$, pyrrolidine $(0.009 \mathrm{ml}, 0.1 \mathrm{mmol})$ and cyclopentanone $(0.009 \mathrm{ml}, 0.1 \mathrm{mmol})$ was irradiated at $160^{\circ} \mathrm{C}$ for $90 \mathrm{~min}$. Purification by flash chromatography on silica gel (petroleum ether:ethyl acetate, 3:1) gave the title compound 5e $(0.018 \mathrm{~g}, 82 \%)$ as a colorless oil; $R_{\mathrm{f}} 0.32$ (petroleum ether:ethyl acetate $\left.1: 1\right) ; v_{\max }($ film) $2961,2924,1708,1597,1416,1375,1260,1193,1021,799 \mathrm{~cm}^{-1} ; \delta_{\mathrm{H}}\left(400 \mathrm{MHz}, \mathrm{CDCl}_{3}\right) 4.42(2 \mathrm{H}, \mathrm{q}, J$ $=7.2 \mathrm{~Hz}), 3.29(2 \mathrm{H}, \mathrm{t}, J=7.6 \mathrm{~Hz}), 2.87(2 \mathrm{H}, \mathrm{t}, J=7.6 \mathrm{~Hz}), 2.56(3 \mathrm{H}, \mathrm{s}), 2.25(3 \mathrm{H}, \mathrm{s}), 2.10(2 \mathrm{H}$, quint, $J=7.6 \mathrm{~Hz}), 1.42(3 \mathrm{H}, \mathrm{t}, J=7.6 \mathrm{~Hz}) ; \delta_{\mathrm{C}}\left(100 \mathrm{MHz}, \mathrm{CDCl}_{3}\right) 166.2(\mathrm{CO}), 154.9(\mathrm{C}), 154.7(\mathrm{C})$, $140.7(\mathrm{C}), 139.9(\mathrm{C}), 131.5(\mathrm{C}), 61.3\left(\mathrm{CH}_{2}\right), 33.1\left(\mathrm{CH}_{2}\right), 31.5\left(\mathrm{CH}_{2}\right), 24.4\left(\mathrm{CH}_{2}\right), 22.5\left(\mathrm{CH}_{3}\right), 15.8$ $\left(\mathrm{CH}_{3}\right), 14.4\left(\mathrm{CH}_{3}\right) ; \mathrm{m} / \mathrm{z}$ (EI) $219\left(60 \%, \mathrm{M}^{+}\right), 147\left(100 \%, \mathrm{M}^{+}-72\right)$ [HRMS (EI) calcd. for $\mathrm{C}_{13} \mathrm{H}_{17} \mathrm{NO}_{2}$ 219.1259. Found 219.1258 (0.2 ppm error)].

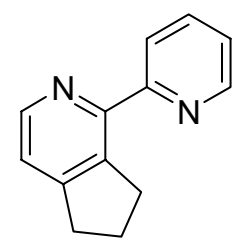

\section{1-Pyridin-2-yl-6,7-dihydro-5H-[2]pyrindine (5f)}

\section{Procedure A:}

A mixture of 3-pyridin-2-yl-[1,2,4]triazine $(0.015 \mathrm{~g}, 0.1 \mathrm{mmol})$, pyrrolidine $(0.009 \mathrm{ml}, 0.1 \mathrm{mmol})$ and cyclopentanone $(0.009 \mathrm{ml}, 0.1 \mathrm{mmol})$ was irradiated at $120^{\circ} \mathrm{C}$ for $15 \mathrm{~min}$. Purification by flash chromatography on silica gel (petroleum ether:ethyl acetate, 1:1) gave the title compound $\mathbf{5 f}(0.016 \mathrm{~g}$, $85 \%$ ) as a colorless oil: $R_{\mathrm{f}} 0.48$ (ethyl acetate); $v_{\max }$ (film) 3055, 2958, 1583, 1570, 1474, 1451, 1252, $1168,1064,903,825,699 \mathrm{~cm}^{-1} ; \delta_{\mathrm{H}}\left(400 \mathrm{MHz}, \mathrm{CDCl}_{3}\right) 8.68(1 \mathrm{H}, \mathrm{ddd}, J=4.8,2.0,0.8 \mathrm{~Hz}), 8.46(1 \mathrm{H}$, 
d, $J=4.8 \mathrm{~Hz}), 8.15(1 \mathrm{H}, \mathrm{ddd}, J=8.0,1.2,0.8 \mathrm{~Hz}), 7.79(1 \mathrm{H}, \mathrm{ddd}, J=8.0,7.2,2.0 \mathrm{~Hz}), 7.26(1 \mathrm{H}$, ddd, $J=7.2,4.8,1.2 \mathrm{~Hz}), 7.21(1 \mathrm{H}, \mathrm{d}, J=4.8 \mathrm{~Hz}), 3.37(2 \mathrm{H}, \mathrm{t}, J=7.6 \mathrm{~Hz}), 2.95(2 \mathrm{H}, \mathrm{t}, J=7.6 \mathrm{~Hz})$, $2.08(2 \mathrm{H}$, quint, $J=7.6 \mathrm{~Hz}) ; \delta_{\mathrm{C}}\left(100 \mathrm{MHz}, \mathrm{CDCl}_{3}\right) 158.2(\mathrm{C}), 155.7(\mathrm{C}), 152.1(\mathrm{C}), 148.6(\mathrm{CH}), 146.8$ $(\mathrm{CH}), 139.2(\mathrm{C}), 136.3(\mathrm{CH}), 122.9(\mathrm{CH}), 122.7(\mathrm{CH}), 119.9(\mathrm{CH}), 32.9\left(\mathrm{CH}_{2}\right), 32.7\left(\mathrm{CH}_{2}\right), 24.9\left(\mathrm{CH}_{2}\right)$; m/z (EI) $196\left(75 \%, \mathrm{M}^{+}\right), 195\left(100 \%, \mathrm{M}^{+}-1\right)$ [HRMS (EI) calcd. for $\mathrm{C}_{13} \mathrm{H}_{12} \mathrm{~N}_{2} 195.0922\left(\mathrm{M}^{+}-1\right)$. Found 195.0915 (3.4 ppm error)].

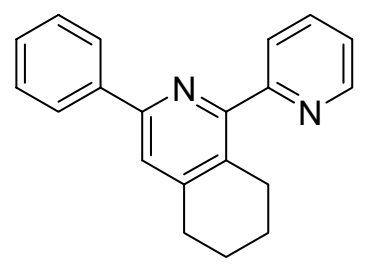

\section{3-Phenyl-1-pyridin-2-yl-5,6,7,8-tetrahydro-isoquinoline (5g)}

\section{Procedure A:}

A mixture of 5-phenyl-3-pyridin-2-yl-[1,2,4]triazine $(0.023 \mathrm{~g}, 0.1 \mathrm{mmol})$, pyrrolidine $(0.009 \mathrm{ml}, 0.1$ mmol $)$ and cyclohexanone $(0.010 \mathrm{ml}, 0.1 \mathrm{mmol})$ was irradiated at $150^{\circ} \mathrm{C}$ for $30 \mathrm{~min}$. Purification by flash chromatography on silica gel (petroleum ether:ethyl acetate, 5:1) gave the title compound $\mathbf{5 g}$ $(0.021 \mathrm{~g}, 75 \%)$.

\section{Procedure B:}

A mixture of 5-phenyl-3-pyridin-2-yl-[1,2,4]triazine $(0.023 \mathrm{~g}, 0.1 \mathrm{mmol}), N$-methylethylenediamine $(0.026 \mathrm{ml}, 0.30 \mathrm{mmol})$, cyclohexanone $(0.060 \mathrm{ml}, 0.6 \mathrm{mmol})$ and powdered $4 \mathrm{~A}$ molecular sieves $(0.100$ g) in toluene $(1.0 \mathrm{ml})$ was heated at $120^{\circ} \mathrm{C}$ for $36 \mathrm{~h}$. Purification by flash chromatography on silica gel (petroleum ether:ethyl acetate, 5:1) gave the title compound $\mathbf{5 g}(0.023 \mathrm{~g}, 79 \%)$.

Yellow oil: $R_{\mathrm{f}} 0.33$ (petroleum ether:ethyl acetate 5:1); $v_{\max }$ (film) 3059, 2929, 2856, 1584, 1563, 1416, $1136,746 \mathrm{~cm}^{-1} ; \delta_{\mathrm{H}}\left(400 \mathrm{MHz}, \mathrm{CDCl}_{3}\right) 8.67(1 \mathrm{H}, \mathrm{ddd}, J=4.8,2.0,0.8 \mathrm{~Hz}), 8.04-8.01(2 \mathrm{H}, \mathrm{m}), 7.87(1$ H, ddd, $J=7.6,1.6,0.8 \mathrm{~Hz}), 7.83(1 \mathrm{H}, \mathrm{td}, J=7.6,2.0 \mathrm{~Hz}), 7.49(1 \mathrm{H}, \mathrm{s}), 7.47-7.42(2 \mathrm{H}, \mathrm{m}), 7.39-7.34$ 
$(1 \mathrm{H}, \mathrm{m}), 7.30(1 \mathrm{H}, \mathrm{ddd}, J=7.6,4.8,1.6 \mathrm{~Hz}), 2.95(2 \mathrm{H}, \mathrm{t}, J=6.4 \mathrm{~Hz}), 2.91(2 \mathrm{H}, \mathrm{t}, J=6.4 \mathrm{~Hz}), 1.90-$ $1.75(4 \mathrm{H}, \mathrm{m}) ; \delta_{\mathrm{C}}\left(100 \mathrm{MHz}, \mathrm{CDCl}_{3}\right) 159.5(\mathrm{C}), 156.1(\mathrm{C}), 153.2(\mathrm{C}), 148.0(\mathrm{C}, \mathrm{CH}), 139.5(\mathrm{C}), 136.5$ $(\mathrm{CH}), 130.7(\mathrm{C}), 128.5(2 \times \mathrm{CH}), 128.3(\mathrm{CH}), 126.8(2 \times \mathrm{CH}), 124.7(\mathrm{CH}), 122.5(\mathrm{CH}), 120.7(\mathrm{CH})$, $29.8\left(\mathrm{CH}_{2}\right), 26.8\left(\mathrm{CH}_{2}\right), 23.1\left(\mathrm{CH}_{2}\right), 22.2\left(\mathrm{CH}_{2}\right) ; \mathrm{m} / \mathrm{z}(\mathrm{EI}) 286\left(85 \%, \mathrm{M}^{+}\right), 285\left(100 \%, \mathrm{M}^{+}-1\right)$ [HRMS (EI) calcd. for $\mathrm{C}_{20} \mathrm{H}_{17} \mathrm{~N}_{2}\left(\mathrm{M}^{+}-1\right)$ 285.1391. Found 285.1388 (1.3 ppm error)].

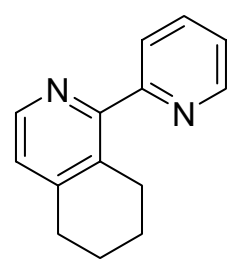

\section{1-Pyridin-2-yl-5,6,7,8-tetrahydro-isoquinoline (5h)}

\section{Procedure A:}

A mixture of 3-pyridin-2-yl-[1,2,4]triazine $(0.015 \mathrm{~g}, 0.1 \mathrm{mmol})$, pyrrolidine $(0.009 \mathrm{ml}, 0.1 \mathrm{mmol})$ and cyclohexanone $(0.010 \mathrm{ml}, 0.1 \mathrm{mmol})$ was irradiated at $150^{\circ} \mathrm{C}$ for $15 \mathrm{~min}$. Purification by flash chromatography on silica gel (petroleum ether:ethyl acetate, 1:1) gave the title compound $\mathbf{5 h}(0.016 \mathrm{~g}$, $75 \%$ ) as a colorless oil: $R_{\mathrm{f}} 0.31$ (ethyl acetate); $v_{\max }$ (film) 2929, 2857, 1581, 1564, 1403, 1130, 1084, 999, $691 \mathrm{~cm}^{-1} ; \delta_{\mathrm{H}}\left(400 \mathrm{MHz}, \mathrm{CDCl}_{3}\right) 8.65(1 \mathrm{H}, \mathrm{ddd}, J=4.8,1.6,0.8 \mathrm{~Hz}), 8.36(1 \mathrm{H}, \mathrm{d}, J=4.8 \mathrm{~Hz})$, $7.88(1 \mathrm{H}, \mathrm{ddd}, J=7.6,7.2,1.6 \mathrm{~Hz}), 7.66(1 \mathrm{H}, \mathrm{ddd}, J=7.6,1.2,0.8 \mathrm{~Hz}), 7.28(1 \mathrm{H}, \mathrm{ddd}, J=7.2,4.8$, $1.2 \mathrm{~Hz}), 7.03(1 \mathrm{H}, \mathrm{d}, J=4.8 \mathrm{~Hz}), 2.88-2.80(4 \mathrm{H}, \mathrm{m}), 1.85-1.70(4 \mathrm{H}, \mathrm{m}) ; \delta_{\mathrm{C}}\left(100 \mathrm{MHz}, \mathrm{CDCl}_{3}\right) 159.0$ (C), $156.5(\mathrm{C}), 148.5(\mathrm{CH}), 147.5(\mathrm{C}), 145.5(\mathrm{CH}), 136.5(\mathrm{CH}), 131.8(\mathrm{C}), 124.2(\mathrm{CH}), 123.9(\mathrm{CH})$, $122.5(\mathrm{CH}), 29.4\left(\mathrm{CH}_{2}\right), 26.8\left(\mathrm{CH}_{2}\right), 22.9\left(\mathrm{CH}_{2}\right), 21.9\left(\mathrm{CH}_{2}\right)$; m/z (EI) $210\left(80 \%, \mathrm{M}^{+}\right), 209\left(100 \%, \mathrm{M}^{+}-\right.$ 1) [HRMS (EI) calcd. for $\mathrm{C}_{14} \mathrm{H}_{14} \mathrm{~N}_{2} 210.1156$. Found 210.1156 (0.4 ppm error)]. 


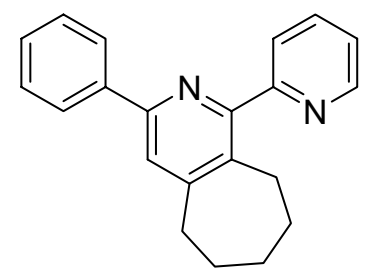

\section{3-Phenyl-1-pyridin-2-yl-6,7,8,9-tetrahydro-5H- \\ cyclohepta $[c]$ pyridine (5i)}

\section{Procedure A:}

A mixture of 5-phenyl-3-pyridin-2-yl-[1,2,4]triazine $(0.023 \mathrm{~g}, 0.1 \mathrm{mmol})$, pyrrolidine $(0.009 \mathrm{ml}, 0.1$ $\mathrm{mmol})$ and cycloheptanone $(0.012 \mathrm{ml}, 0.1 \mathrm{mmol})$ was irradiated at $120^{\circ} \mathrm{C}$ for $30 \mathrm{~min}$. Purification by flash chromatography on silica gel (petroleum ether:ethyl acetate, 5:1) gave the title compound 5i $(0.027 \mathrm{~g}, 91 \%)$.

\section{Procedure B:}

A mixture of 5-phenyl-3-pyridin-2-yl-[1,2,4]triazine $(0.023 \mathrm{~g}, 0.1 \mathrm{mmol}), N$-methylethylenediamine $(0.026 \mathrm{ml}, 0.30 \mathrm{mmol})$, cycloheptanone $(0.072 \mathrm{ml}, 0.6 \mathrm{mmol})$ and powdered $4 \mathrm{~A}$ molecular sieves $(0.100 \mathrm{~g})$ in toluene $(1.0 \mathrm{ml})$ was heated at $120^{\circ} \mathrm{C}$ for $22 \mathrm{~h}$. Purification by flash chromatography on silica gel (petroleum ether:ethyl acetate, 5:1) gave the title compound $\mathbf{5 i}(0.030 \mathrm{~g}, 100 \%)$.

Colorless oil: $R_{\mathrm{f}} 0.28$ (petroleum ether:ethyl acetate 5:1); $v_{\max }($ film) 2962, 2924, 2852, 1584, 1261, $1093,1020,801 \mathrm{~cm}^{-1} ; \delta_{\mathrm{H}}\left(400 \mathrm{MHz}, \mathrm{CDCl}_{3}\right) 8.69(1 \mathrm{H}, \mathrm{ddd}, J=4.8,1.6,0.8 \mathrm{~Hz}), 8.04-8.00(2 \mathrm{H}, \mathrm{m})$, $7.82(1 \mathrm{H}, \mathrm{td}, J=7.6,1.6 \mathrm{~Hz}), 7.78(1 \mathrm{H}, \mathrm{ddd}, J=7.6,1.2,0.8 \mathrm{~Hz}), 7.53(1 \mathrm{H}, \mathrm{s}), 7.47-7.41(2 \mathrm{H}, \mathrm{m})$, 7.39-7.34 (1 H, m), 7.30 (1 H, ddd, $J=7.6,4.8,1.2 \mathrm{~Hz}), 2.98-2.91$ (4 H, m), 1.92-1.83 (2 H, m), 1.78$1.70(2 \mathrm{H}, \mathrm{m}), 1.70-1.62(2 \mathrm{H}, \mathrm{m}) ; \delta_{\mathrm{C}}\left(100 \mathrm{MHz}, \mathrm{CDCl}_{3}\right) 159.9(\mathrm{C}), 155.9(\mathrm{C}), 145.5(\mathrm{C}), 154.1(\mathrm{C})$, $148.3(\mathrm{CH}), 139.5(\mathrm{C}), 136.4(\mathrm{CH}), 135.9(\mathrm{C}), 128.5(2 \times \mathrm{CH}), 128.4(\mathrm{CH}), 126.8(2 \times \mathrm{CH}), 124.7$ (CH), $122.3(\mathrm{CH}), 120.7(\mathrm{CH}), 36.6\left(\mathrm{CH}_{2}\right), 32.3\left(\mathrm{CH}_{2}\right), 29.9\left(\mathrm{CH}_{2}\right), 27.6\left(\mathrm{CH}_{2}\right), 27.5\left(\mathrm{CH}_{2}\right) ; \mathrm{m} / \mathrm{z}(\mathrm{EI})$ $300\left(80 \%, \mathrm{M}^{+}\right), 271\left(100 \%, \mathrm{M}^{+}-29\right)$ [HRMS (EI) calcd. for $\mathrm{C}_{21} \mathrm{H}_{19} \mathrm{~N}_{2} 299.1548\left(\mathrm{M}^{+}-1\right)$. Found 299.1542 (2.0 ppm error)]. 


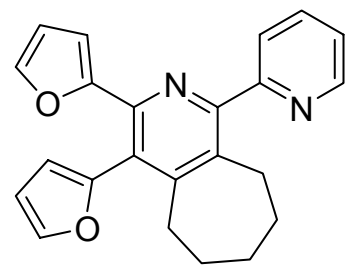

\section{3,4-Di-furan-2-yl-1-pyridin-2-yl-6,7,8,9-tetrahydro-}

$5 H$-cyclohepta $[c]$ pyridine $(5 \mathbf{j})$

\section{Procedure A:}

A mixture of 5,6-difuran-2-yl-3-pyridin-2-yl-[1,2,4]triazine $(0.029 \mathrm{~g}, 0.1 \mathrm{mmol})$, pyrrolidine $(0.009 \mathrm{ml}$, $0.1 \mathrm{mmol})$ and cycloheptanone $(0.012 \mathrm{ml}, 0.1 \mathrm{mmol})$ was irradiated at $120^{\circ} \mathrm{C}$ for $30 \mathrm{~min}$. Purification by flash chromatography on silica gel (petroleum ether:ethyl acetate, 4:1) gave the title compound $\mathbf{5 j}$ $(0.024 \mathrm{~g}, 69 \%)$ as a colourless oil: $R_{\mathrm{f}} 0.53$ (petroleum ether:ethyl acetate 1:1) gave the title compound; $v_{\max }($ film $) 2923,1588,1455,1210,1090,792,737 \mathrm{~cm}^{-1} ; \delta_{\mathrm{H}}\left(400 \mathrm{MHz}, \mathrm{CDCl}_{3}\right) 8.69(1 \mathrm{H}, \mathrm{ddd}, J=4.8$, 2.0, $0.8 \mathrm{~Hz}), 7.83(1 \mathrm{H}, \mathrm{td}, J=7.6,2.0 \mathrm{~Hz}), 7.77(1 \mathrm{H}, \mathrm{ddd}, J=7.6,1.2,0.8 \mathrm{~Hz}), 7.60(1 \mathrm{H}, \mathrm{dd}, J=2.0$, $0.8 \mathrm{~Hz}), 7.42(1 \mathrm{H}, \mathrm{dd}, J=2.0,0.8 \mathrm{~Hz}), 7.31(1 \mathrm{H}, \mathrm{ddd}, J=7.6,4.8,1.2 \mathrm{~Hz}), 6.55(1 \mathrm{H}, \mathrm{dd}, J=3.2,2.0$ Hz), 6.31-6.27 (2 H, m) $5.72(1 \mathrm{H}, \mathrm{dd}, J=3.2,0.8 \mathrm{~Hz}), 2.98-2.92(2 \mathrm{H}, \mathrm{m}), 2.73-2.68(2 \mathrm{H}, \mathrm{m}), 1.86-$ $1.59(6 \mathrm{H}, \mathrm{m}) ; \delta_{\mathrm{C}}\left(100 \mathrm{MHz}, \mathrm{CDCl}_{3}\right) 159.2(\mathrm{C}), 156.2(\mathrm{C}), 155.8(\mathrm{C}), 151.9(\mathrm{C}), 149.9(\mathrm{C}), 148.5(\mathrm{CH})$, $146.5(\mathrm{C}), 143.1(\mathrm{CH}), 142.2(\mathrm{CH}), 136.7(\mathrm{CH}), 135.7(\mathrm{C}), 124.8(\mathrm{CH}), 122.7(\mathrm{CH}), 122.3(\mathrm{C}), 111.3$ (2 x CH), $110.5(\mathrm{CH}), 109.9(\mathrm{CH}), 31.8\left(\mathrm{CH}_{2}\right), 31.5\left(\mathrm{CH}_{2}\right), 29.9\left(\mathrm{CH}_{2}\right), 27.4\left(\mathrm{CH}_{2}\right), 26.8\left(\mathrm{CH}_{2}\right) ; \mathrm{m} / \mathrm{z}$ (EI) $356\left(100 \%, \mathrm{M}^{+}\right)$[HRMS (EI) calcd. for $\mathrm{C}_{23} \mathrm{H}_{19} \mathrm{~N}_{2} \mathrm{O}_{2} 355.1446\left(\mathrm{M}^{+}-1\right)$. Found $355.1439(2.0 \mathrm{ppm}$ error)]. 


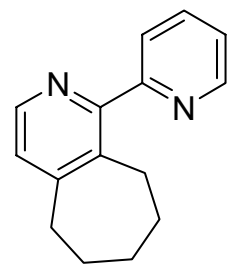

1-Pyridin-2-yl-6,7,8,9-tetrahydro-5H-cyclohepta[c]pyridine (5k)

\section{Procedure A:}

A mixture of 3-pyridin-2-yl-[1,2,4]triazine $(0.015 \mathrm{~g}, 0.1 \mathrm{mmol})$, pyrrolidine $(0.009 \mathrm{ml}, 0.1 \mathrm{mmol})$ and cycloheptanone $(0.012 \mathrm{ml}, 0.1 \mathrm{mmol})$ was irradiated at $120^{\circ} \mathrm{C}$ for $15 \mathrm{~min}$. Purification by flash chromatography on silica gel (petroleum ether:ethyl acetate, $1: 1)$ gave the title compound $\mathbf{5 k}(0.018 \mathrm{~g}$, $82 \%$ ) as a colorless oil: $R_{\mathrm{f}} 0.23$ (ethyl acetate); $v_{\max }$ (film) 3050, 2924, 2851, 1580, 1563, 1438, 1325 , $1194,1147,958,745 \mathrm{~cm}^{-1} ; \delta_{\mathrm{H}}\left(400 \mathrm{MHz}, \mathrm{CDCl}_{3}\right) 8.67(1 \mathrm{H}, \mathrm{ddd}, J=4.8,1.6,0.8 \mathrm{~Hz}), 8.37(1 \mathrm{H}, \mathrm{d}, J=$ $4.8 \mathrm{~Hz}), 7.78(1 \mathrm{H}, \mathrm{td}, J=7.6,1.6 \mathrm{~Hz}), 7.60(1 \mathrm{H}, \mathrm{ddd}, J=7.6,1.2,0.8 \mathrm{~Hz}), 7.28(1 \mathrm{H}, \mathrm{ddd}, J=7.6$, 4.8, $1.2 \mathrm{~Hz}), 7.07(1 \mathrm{H}, \mathrm{d}, J=4.8 \mathrm{~Hz}), 2.91-2.84(4 \mathrm{H}, \mathrm{m}), 1.88-1.81(2 \mathrm{H}, \mathrm{m}), 1.73-1.60(4 \mathrm{H}, \mathrm{m}) ; \delta_{\mathrm{C}}$ $\left(100 \mathrm{MHz}, \mathrm{CDCl}_{3}\right) 159.4(\mathrm{C}), 156.1(\mathrm{C}), 153.9(\mathrm{C}), 148.8(\mathrm{CH}), 146.6(\mathrm{CH}), 137.2(\mathrm{C}), 136.4(\mathrm{CH})$, $124.3(\mathrm{CH}), 123.9(\mathrm{CH}), 122.3(\mathrm{CH}), 36.2\left(\mathrm{CH}_{2}\right), 32.3\left(\mathrm{CH}_{2}\right), 30.1\left(\mathrm{CH}_{2}\right), 27.4\left(\mathrm{CH}_{2}\right), 27.3\left(\mathrm{CH}_{2}\right) ; \mathrm{m} / \mathrm{z}$ (EI) $224\left(65 \%, \mathrm{M}^{+}\right), 195\left(100 \%, \mathrm{M}^{+}-29\right)$ [HRMS (EI) calcd. for $\mathrm{C}_{15} \mathrm{H}_{16} \mathrm{~N}_{2}$ 224.1313. Found 224.1305 (3.5 ppm error)]. 


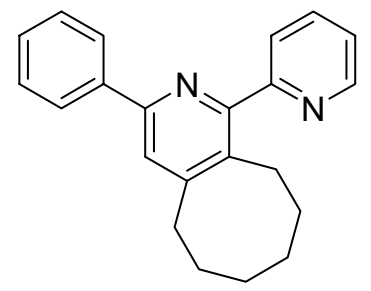

\section{3-Phenyl-1-pyridin-2-yl-5,6,7,8,9,10-hexahydro- cycloocta $[c]$ pyridine (5l)}

\section{Procedure A:}

A mixture of 5-phenyl-3-pyridin-2-yl-[1,2,4]triazine $(0.023 \mathrm{~g}, 0.1 \mathrm{mmol})$, pyrrolidine $(0.009 \mathrm{ml}, 0.1$ $\mathrm{mmol})$ and cyclooctanone $(0.013 \mathrm{ml}, 0.1 \mathrm{mmol})$ was irradiated at $120^{\circ} \mathrm{C}$ for $30 \mathrm{~min}$. Purification by flash chromatography on silica gel (petroleum ether:ethyl acetate, 5:1) gave the title compound $\mathbf{5}$ $(0.025 \mathrm{~g}, 80 \%)$.

\section{Procedure B:}

A mixture of 5-phenyl-3-pyridin-2-yl-[1,2,4]triazine $(0.023 \mathrm{~g}, 0.1 \mathrm{mmol}), \mathrm{N}$-methylethylenediamine $(0.026 \mathrm{ml}, 0.30 \mathrm{mmol})$, cyclooctanone $(0.078 \mathrm{ml}, 0.6 \mathrm{mmol})$ and powdered $4 \mathrm{~A}$ molecular sieves $(0.100$ g) in toluene $(1.0 \mathrm{ml})$ was heated at $120^{\circ} \mathrm{C}$ for $21 \mathrm{~h}$. Purification by flash chromatography on silica gel (petroleum ether:ethyl acetate, 5:1) gave the title compound $\mathbf{5 l}(0.024 \mathrm{~g}, 77 \%)$.

Colorless oil: $R_{\mathrm{f}} 0.40$ (petroleum ether:ethyl acetate, 5:1); $v_{\max }($ film) 3060, 2923, 2853, 1585, 15.52, $1496,1260,993,665 \mathrm{~cm}^{-1} ; \delta_{\mathrm{H}}\left(400 \mathrm{MHz}, \mathrm{CDCl}_{3}\right) 8.67(1 \mathrm{H}, \mathrm{ddd}, J=4.8,1.6,1.2 \mathrm{~Hz}), 8.05(2 \mathrm{H}, \mathrm{dd}, J$ $=8.0,1.2 \mathrm{~Hz}), 7.81(1 \mathrm{H}, \mathrm{td}, J=7.6,1.6 \mathrm{~Hz}), 7.77(1 \mathrm{H}, \mathrm{ddd}, J=7.6,2.0,1.2 \mathrm{~Hz}), 7.55(1 \mathrm{H}, \mathrm{s}), 7.48-$ $7.41(2 \mathrm{H}, \mathrm{m}), 7.40-7.35(1 \mathrm{H}, \mathrm{m}), 7.30(1 \mathrm{H}, \mathrm{ddd}, J=7.6,4.8,2.0 \mathrm{~Hz}), 3.02-2.97(2 \mathrm{H}, \mathrm{m}), 2.92-2.87$ (2 H, m), 1.84-1.32 (8 H, m); $\delta_{\mathrm{C}}\left(100 \mathrm{MHz}, \mathrm{CDCl}_{3}\right) 159.9$ (C), 156.3 (C), 154.0 (C), $152.8(\mathrm{C}), 148.1$ $(\mathrm{CH}), 139.4(\mathrm{C}), 136.4(\mathrm{CH}), 133.7(\mathrm{C}), 128.5(2 \times \mathrm{CH}), 128.4(\mathrm{CH}), 126.9(2 \times \mathrm{CH}), 124.6(\mathrm{CH})$, $122.4(\mathrm{CH}), 120.8(\mathrm{CH}), 32.8\left(\mathrm{CH}_{2}\right), 31.9\left(\mathrm{CH}_{2}\right), 30.8\left(\mathrm{CH}_{2}\right), 26.2\left(\mathrm{CH}_{2}\right), 26.1\left(\mathrm{CH}_{2}\right), 25.7\left(\mathrm{CH}_{2}\right) ; \mathrm{m} / \mathrm{z}$ (EI) $314\left(98 \%, \mathrm{M}^{+}\right), 271\left(100 \%, \mathrm{M}^{+}-29\right)$ [HRMS (EI) calcd. for $\mathrm{C}_{22} \mathrm{H}_{22} \mathrm{~N}_{2}$ 314.1782. Found 314.1781 (0.4 ppm error)]. 


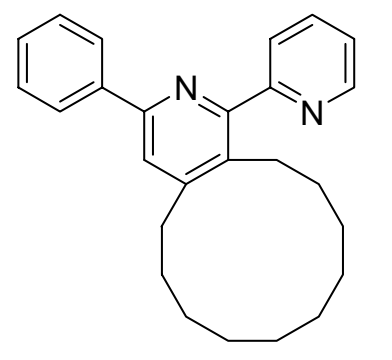

$$
\begin{aligned}
& \text { 3-Phenyl-1-pyridin-2-yl-5,6,7,8,9,10,11,12,13,14- } \\
& \text { decahydro-cyclododeca[c]pyridine (5m) }
\end{aligned}
$$

\section{Procedure A:}

A mixture of 5-phenyl-3-pyridin-2-yl-[1,2,4]triazine $(0.023 \mathrm{~g}, 0.1 \mathrm{mmol})$, pyrrolidine $(0.009 \mathrm{ml}, 0.1$ $\mathrm{mmol})$ and cyclododecanone $(0.018 \mathrm{~g}, 0.1 \mathrm{mmol})$ was irradiated at $150^{\circ} \mathrm{C}$ for $30 \mathrm{~min}$. Purification by flash chromatography on silica gel (petroleum ether:ethyl acetate 5:1) gave the title compound 5m $(0.029 \mathrm{~g}, 81 \%)$ as a colourless oil: $R_{\mathrm{f}} 0.40$ (petroleum ether:ethyl acetate, $\left.5: 1\right) ; v_{\max }$ (film) 2923, 2852, $1584,1546,1378,1261,763 \mathrm{~cm}^{-1} ; \delta_{\mathrm{H}}\left(400 \mathrm{MHz}, \mathrm{CDCl}_{3}\right) 8.65(1 \mathrm{H}, \mathrm{ddd}, J=4.8,2.0,0.8 \mathrm{~Hz}), 8.02-$ $7.99(2 \mathrm{H}, \mathrm{m}), 7.83(1 \mathrm{H}, \mathrm{td}, J=7.6,2.0 \mathrm{~Hz}), 7.76(1 \mathrm{H}, \mathrm{ddd}, J=7.6,1.2,0.8 \mathrm{~Hz}), 7.60(1 \mathrm{H}, \mathrm{s}), 7.46-$ $7.41(2 \mathrm{H}, \mathrm{m}), 7.39-7.34(1 \mathrm{H}, \mathrm{m}), 7.31(1 \mathrm{H}, \mathrm{ddd}, J=7.6,4.8,1.2 \mathrm{~Hz}), 3.02-2.96(2 \mathrm{H}, \mathrm{m}), 2.82-2.76$ (2 H, m), 1.88-0.80 (16 H, m); $\delta_{\mathrm{C}}\left(100 \mathrm{MHz}, \mathrm{CDCl}_{3}\right) 160.3(\mathrm{C}), 157.2(\mathrm{C}), 153.6(\mathrm{C}), 152.1(\mathrm{C}), 148.1$ $(\mathrm{CH}), 139.5(\mathrm{C}), 136.6(\mathrm{CH}), 133.9(\mathrm{C}), 128.5(2 \times \mathrm{CH}), 128.4(\mathrm{CH}), 126.8(2 \times \mathrm{CH}), 124.6(\mathrm{CH})$, $122.5(\mathrm{CH}), 121.3(\mathrm{CH}), 30.0\left(\mathrm{CH}_{2}\right), 29.4\left(\mathrm{CH}_{2}\right), 28.4\left(\mathrm{CH}_{2}\right), 27.1\left(2 \times \mathrm{CH}_{2}\right), 26.3\left(\mathrm{CH}_{2}\right), 26.1\left(\mathrm{CH}_{2}\right)$, $25.2\left(\mathrm{CH}_{2}\right), 22.9\left(\mathrm{CH}_{2}\right), 22.7\left(\mathrm{CH}_{2}\right) ; \mathrm{m} / \mathrm{z}(\mathrm{EI}) 370\left(100 \%, \mathrm{M}^{+}\right)$[HRMS (EI) calcd. for $\mathrm{C}_{26} \mathrm{H}_{30} \mathrm{~N}_{2}$ 370.2408. Found 370.2423 (-3.9 ppm error)]. 


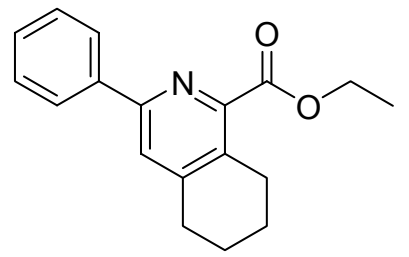

3-Phenyl-5,6,7,8-tetrahydro-isoquinoline-1-

carboxylic acid ethyl ester (5n)

\section{Procedure B:}

A mixture of 5-phenyl-[1,2,4]triazine-3-carboxylic acid ethyl ester $(0.023 \mathrm{~g}, 0.1 \mathrm{mmol}), \mathrm{N}$ methylethylenediamine $(0.026 \mathrm{ml}, 0.30 \mathrm{mmol})$, cyclohexanone $(0.060 \mathrm{ml}, 0.6 \mathrm{mmol})$ and powdered $4 \mathrm{~A}$ molecular sieves $(0.100 \mathrm{~g})$ in toluene $(1.0 \mathrm{ml})$ was heated at $120^{\circ} \mathrm{C}$ for $16 \mathrm{~h}$. Purification by flash chromatography on silica gel (petroleum ether:ethyl acetate $4: 1)$ gave the title compound $\mathbf{5 n}(0.017 \mathrm{~g}$, $61 \%$ ) as a colorless oil; $R_{\mathrm{f}} 0.48$ (petroleum ether:ethyl acetate, 1:1); $v_{\max }$ (film) 2933, 2859, 1731, 1591, $1438,1204,1153,1025,694 \mathrm{~cm}^{-1} ; \delta_{\mathrm{H}}\left(400 \mathrm{MHz}, \mathrm{CDCl}_{3}\right)$ 8.00-7.95 $(2 \mathrm{H}, \mathrm{m}), 7.52(1 \mathrm{H}, \mathrm{s}), 7.47-7.42(2$ H, m), 7.41-7.35 (1 H, m), 4.46 (2 H, q, $J=7.6 \mathrm{~Hz}), 2.99-2.84(4 \mathrm{H}, \mathrm{m}), 1.87-1.80(4 \mathrm{H}, \mathrm{m}), 1.44(3 \mathrm{H}$, t, $J=7.6 \mathrm{~Hz}) ; \delta_{\mathrm{C}}\left(100 \mathrm{MHz}, \mathrm{CDCl}_{3}\right) 167.4(\mathrm{CO}), 153.9(\mathrm{C}), 149.3(\mathrm{C}), 148.6(\mathrm{C}), 138.9(\mathrm{C}), 131.4(\mathrm{C})$, 128. $9(\mathrm{CH}), 128.8(2 \times \mathrm{CH}), 127.0(2 \times \mathrm{CH}), 122.9(\mathrm{CH}), 61.6\left(\mathrm{CH}_{2}\right), 29.9\left(\mathrm{CH}_{2}\right), 25.8\left(\mathrm{CH}_{2}\right), 22.8$ $\left(\mathrm{CH}_{2}\right), 22.1\left(\mathrm{CH}_{2}\right), 14.4\left(\mathrm{CH}_{3}\right) ; \mathrm{m} / \mathrm{z}(\mathrm{CI}): 282\left(\mathrm{MH}^{+}, 100 \%\right)$ [HRMS (CI): calcd. for m/z (CI): 282 $\left(\mathrm{MH}^{+}, 100 \%\right)$ [HRMS (CI): calcd. for $\mathrm{C}_{16} \mathrm{H}_{18} \mathrm{~N}_{4} \mathrm{O}$ 282.1480. Found 282.1489 (-3.1 ppm error)]. 


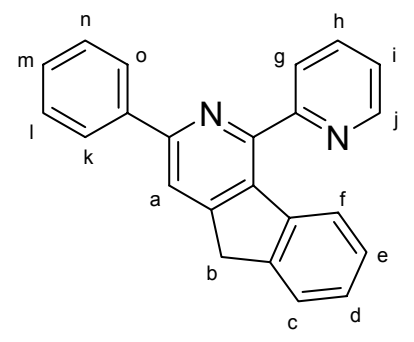

2-Phenyl-4-pyridin-2-yl-9H-3-aza-fluorene (8a)

\section{Procedure A:}

A mixture of 5-phenyl-3-pyridin-2-yl-[1,2,4]triazine $(0.023 \mathrm{~g}, 0.1 \mathrm{mmol})$, pyrrolidine $(0.018 \mathrm{ml}, 0.2$ $\mathrm{mmol})$ and 1-indanone $(0.026 \mathrm{~g}, 0.2 \mathrm{mmol})$ was irradiated at $120^{\circ} \mathrm{C}$ for $30 \mathrm{~min}$. Purification by flash chromatography on silica gel (petroleum ether:ethyl acetate, $4: 1)$ gave the title compound $\mathbf{8 a}(0.021 \mathrm{~g}$, $66 \%$ ) as a white solid: $\mathrm{mp} 115-116{ }^{\circ} \mathrm{C}$ (hexane- $\mathrm{CHCl}_{3}$ ); $v_{\max }$ (film) 3069, 2935, 1598, 1549, 1475, $1392,1264,1102,837 \mathrm{~cm}^{-1} ; \delta_{\mathrm{H}}\left(400 \mathrm{MHz}, \mathrm{CDCl}_{3}\right) 8.81(1 \mathrm{H}, \mathrm{ddd}, J=4.8,2.0,0.8 \mathrm{~Hz}, \mathrm{H}-\mathrm{j}), 8.17-8.13$ (2 H, m, H-k,o), 8.03 (1 H, ddd, J= 7.6, 1.2, $0.8 \mathrm{~Hz}, \mathrm{H}-\mathrm{g}), 7.98$ (1 H, s, H-a), 7.95 (1 H, td, J = 7.6, 2.0 Hz, H-h), 7.56-7.39 (6 H, m, H-c,f,i,1,m,n), 7.32 (1 H, td, J= 7.6, $1.2 \mathrm{~Hz}, \mathrm{H}-\mathrm{e}), 7.22$ (1 H, td, $J=7.6$, $1.2 \mathrm{~Hz}, \mathrm{H}-\mathrm{d}), 3.96$ (2 H, s, H-b); $\delta_{\mathrm{C}}\left(100 \mathrm{MHz}, \mathrm{CDCl}_{3}\right) 158.8$ (C), 154.8 (C), 153.9 (C), 152.0 (C), $148.6(\mathrm{CH}), 143.0(\mathrm{C}), 139.3(\mathrm{C}), 138.9(\mathrm{C}), 137.1(\mathrm{CH}), 134.6(\mathrm{C}), 128.7(\mathrm{CH}), 128.6(2 \times \mathrm{CH}), 127.5$ $(\mathrm{CH}), 126.9(2 \times \mathrm{CH}), 126.7(\mathrm{CH}), 124.7(\mathrm{CH}), 124.5(\mathrm{CH}), 124.0(\mathrm{CH}), 123.5(\mathrm{CH}), 116.5(\mathrm{CH}), 37.2$ $\left(\mathrm{CH}_{2}\right) ; \mathrm{m} / \mathrm{z}(\mathrm{EI}) 320\left(40 \%, \mathrm{M}^{+}\right), 319\left(100 \%, \mathrm{M}^{+}-1\right)$ [HRMS (EI) calcd. for $\mathrm{C}_{23} \mathrm{H}_{15} \mathrm{~N}_{2} 319.1235\left(\mathrm{M}^{+}-1\right)$. Found 319.1238 (-1.1 ppm error)]. 
<smiles>c1ccc(-c2cc3c(c(-c4ccccn4)n2)-c2ccccc2CC3)cc1</smiles><smiles>Ic1ccccc1-c1cc2c(c(-c3ccccn3)n1)CCc1ccccc1-2</smiles>

\section{2-Phenyl-4-pyridin-2-yl-5,6-dihydro-benzo[f]isoquinoline (9b)}

\section{Procedure A:}

Irradiation of 5-phenyl-3-pyridin-2-yl-[1,2,4]triazine (0.023 g, $0.1 \mathrm{mmol})$, pyrrolidine $(0.018 \mathrm{ml}, 0.2$ $\mathrm{mmol})$ and $\alpha$-tetralone $(0.029 \mathrm{~g}, 0.2 \mathrm{mmol})$ at $150^{\circ} \mathrm{C}$ for $60 \mathrm{~min}$. gave the mixture of $\mathbf{8 b}$ and $9 \mathbf{b}(0.014$ g, 40\% combined yield, 2:1, 8b:9b from 'H NMR) which was separated by flash chromatography on silica gel (petroleum ether:ethyl acetate, 5:1).

For 8b. Colorless oil. $R_{\mathrm{f}} 0.17$ (petroleum ether:ethyl acetate 5:1); $v_{\max }$ (film) 3057, 2925, 2852, 1585 , $1560,1521,1457,1375,1150,909,694 \mathrm{~cm}^{-1} ; \delta_{\mathrm{H}}\left(400 \mathrm{MHz}, \mathrm{CDCl}_{3}\right) 8.60(1 \mathrm{H}, \mathrm{ddd}, J=4.8,2.0,0.8$ Hz, H-k), 8.12-8.08 (2 H, m, H-1,p), 7.82 (1 H, td, J= 7.2, 2.0 Hz, H-i), 7.77 (1 H, ddd, J= 7.2, 1.2, 0.8 Hz, H-h), 7.68 (1 H, s, H-a), 7.50-7.44 (2 H, m, H-m,o), 7.43-7.38 (1 H, m, H-n), 7.31 (1 H, ddd, J= 7.2, 4.8, 1.2 Hz, H-j), 7.24 (1 H, dd, $J=7.6,1.2 \mathrm{~Hz}, \mathrm{H}-\mathrm{g}), 7.12$ (1 H, ddd, J = 7.6, 7.2, 1.2 Hz, H-e), 6.88 (1 H, ddd, $J=7.6,7.2,1.2 \mathrm{~Hz}, \mathrm{H}-\mathrm{f}), 6.63$ (1 H, dd, $J=7.6,1.2 \mathrm{~Hz}, \mathrm{H}-\mathrm{d}), 3.00-2.90$ (4 H, m, Hb,c); $\delta_{\mathrm{C}}\left(100 \mathrm{MHz}, \mathrm{CDCl}_{3}\right) 159.97(\mathrm{C}), 154.4(\mathrm{C}), 149.8(\mathrm{C}), 149.1(\mathrm{C}), 138.9(\mathrm{CH}), 138.7$ (C), 136.7 (2 x C), $131.9(\mathrm{CH}), 128.9(\mathrm{C}), 128,8(\mathrm{CH}), 128.7(\mathrm{CH}), 128.6(2 \times \mathrm{CH}), 127.7(\mathrm{CH}), 127.2(\mathrm{CH})$, $126.9(2 \times \mathrm{CH}), 125.7(\mathrm{CH}), 124.7(\mathrm{CH}), 122.8(\mathrm{CH}), 118.9(\mathrm{CH}), 29.7\left(\mathrm{CH}_{2}\right), 28.7(\mathrm{CH}) ; \mathrm{m} / \mathrm{z}(\mathrm{EI})$ $334\left(30 \%, \mathrm{M}^{+}\right), 333\left(100 \%, \mathrm{M}^{+}-1\right)$ [HRMS (EI) calcd. for $\mathrm{C}_{24} \mathrm{H}_{17} \mathrm{~N}_{2} 333.1391\left(\mathrm{M}^{+}-1\right)$. Found 333.1389 (0.6 ppm error)]. 
For 9b. Colorless oil. $R_{\mathrm{f}} 0.33$ (petroleum ether:ethyl acetate, 5:1); $v_{\max }$ (film) 2923, 2852, 1652, 1585, $1520,1489,1436,1376,1074,631 \mathrm{~cm}^{-1} ; \delta_{\mathrm{H}}\left(400 \mathrm{MHz}, \mathrm{CDCl}_{3}\right) 8.71(1 \mathrm{H}, \mathrm{ddd}, J=4.8,1.6,0.8 \mathrm{~Hz}, \mathrm{H}-$ k), 8.15-8.11 (2 H, m, H-l,p), 8.10 (1 H, s, H-a), 8.03 (1 H, ddd, J= 7.6, 1.2, 0.8 Hz, H-h), 7.94-7.91 (1 H, dd, $J=7.2,1.6 \mathrm{~Hz}, \mathrm{H}-\mathrm{b}), 7.88(1 \mathrm{H}, \mathrm{td}, J=7.6,1.6 \mathrm{~Hz}, \mathrm{H}-\mathrm{i}), 7.53-7.47$ (2 H, m, H-m,o), 7.45-7.28 (5 H, m, H-c,d,e,j,n), 3.20-3.14 (2 H, m, H-g), 2.88-2.82 (2 H, m, H-f); $\delta_{\mathrm{C}}\left(100 \mathrm{MHz}, \mathrm{CDCl}_{3}\right) 159.1$ (C), $155.4(\mathrm{C}), 155.0(\mathrm{C}), 148.2(\mathrm{CH}), 144.0$ (C), 139.7 (C), 138.5 (C), $136.5(\mathrm{CH}), 132.7$ (C), 129.6 (C), $129.3(\mathrm{CH}), 128.7(3 \times \mathrm{CH}), 128.2(\mathrm{CH}), 127.1(\mathrm{CH}), 126.9(2 \times \mathrm{CH}), 124.8(\mathrm{CH}), 124.5(\mathrm{CH})$, $122.7(\mathrm{CH}), 114.8(\mathrm{CH}), 28.6\left(\mathrm{CH}_{2}\right), 24.8\left(\mathrm{CH}_{2}\right) ; \mathrm{m} / \mathrm{z}(\mathrm{EI}) 334\left(60 \%, \mathrm{M}^{+}\right), 333\left(100 \%, \mathrm{M}^{+}-1\right)$ [HRMS (EI) calcd. for $\mathrm{C}_{24} \mathrm{H}_{17} \mathrm{~N}_{2} 333.1391\left(\mathrm{M}^{+}-1\right)$. Found 333.1385 (1.9 ppm error)].

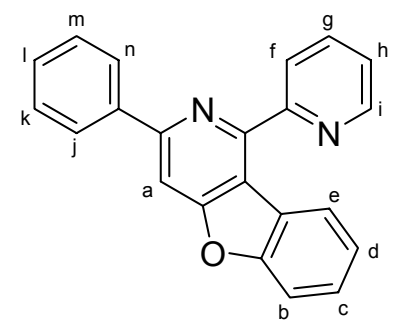

\section{3-Phenyl-1-pyridin-2-yl-benzo[4,5] furo[3,2-c]pyridine (8c)}

\section{Procedure A:}

A mixture of 5-phenyl-3-pyridin-2-yl-[1,2,4]triazine $(0.023 \mathrm{~g}, 0.1 \mathrm{mmol})$, pyrrolidine $(0.018 \mathrm{ml}, 0.2$ $\mathrm{mmol})$ and 3-coumarone $(0.026 \mathrm{~g}, 0.2 \mathrm{mmol})$ was irradiated at $150^{\circ} \mathrm{C}$ for $60 \mathrm{~min}$. Purification by flash chromatography on silica gel (petroleum ether:ethyl acetate, 5:1) gave the title compound $\mathbf{8 c}(0.021 \mathrm{~g}$, $66 \%$ ) as a white solid: $\mathrm{mp} 87-88^{\circ} \mathrm{C}$ (hexane- $\mathrm{CHCl}_{3}$ ); $v_{\max }$ (film) $3061,1593,1416,1380,1185,951$, 767, $692 \mathrm{~cm}^{-1} ; \delta_{\mathrm{H}}\left(400 \mathrm{MHz}, \mathrm{CDCl}_{3}\right) 8.91(1 \mathrm{H}, \mathrm{ddd}, J=4.8,2.0,0.8 \mathrm{~Hz}, \mathrm{H}-\mathrm{i}), 8.76(1 \mathrm{H}, \mathrm{dd}, J=8.0$, 1.6 Hz, H-e), 8.52 (1 H, ddd, $J=7.6,1.2,0.8 \mathrm{~Hz}, \mathrm{H}-\mathrm{f}), 8.25-8.20$ (2 H, m, H-j,n), 7.96 (1 H, s, H-a), $7.95(1 \mathrm{H}, \mathrm{td}, J=7.6,2.0 \mathrm{~Hz}, \mathrm{H}-\mathrm{g}), 7.61(1 \mathrm{H}, \mathrm{dd}, J=8.4,1.6 \mathrm{~Hz}, \mathrm{H}-\mathrm{b}), 7.57-7.50(3 \mathrm{H}, \mathrm{m}, \mathrm{H}-\mathrm{c}, \mathrm{k}, \mathrm{m})$, 7.49-7.43 (2 H, m, H-h,l), $7.38(1 \mathrm{H}, \mathrm{ddd}, J=8.0,7.6,1.6 \mathrm{~Hz}, \mathrm{H}-\mathrm{d}) ; \delta_{\mathrm{C}}\left(100 \mathrm{MHz}, \mathrm{CDCl}_{3}\right) 163.8(\mathrm{C})$, 
$157.6(\mathrm{C}), 156.7(\mathrm{C}), 154.3(\mathrm{C}), 152.4(\mathrm{C}), 148.3(\mathrm{CH}), 138.9(\mathrm{C}), 136.9(\mathrm{CH}), 129.0(\mathrm{CH}), 128.7(2 \times$ $\mathrm{CH}), 128.3(\mathrm{CH}), 127.0(2 \times \mathrm{CH}), 126.2(\mathrm{CH}), 124.1(\mathrm{CH}), 123.9(\mathrm{CH}), 123.4(\mathrm{CH}), 122.0(\mathrm{C}), 118.5$ (C), $111.2(\mathrm{CH}), 103.2(\mathrm{CH}) ; \mathrm{m} / \mathrm{z}(\mathrm{EI}) 322\left(55 \%, \mathrm{M}^{+}\right), 321\left(100 \%, \mathrm{M}^{+}-1\right)$ [HRMS (EI) calcd. for $\mathrm{C}_{22} \mathrm{H}_{13} \mathrm{~N}_{2} \mathrm{O} 321.1027\left(\mathrm{M}^{+}-1\right)$. Found 321.1031 (-1.1 ppm error)].<smiles>c1ccc(-c2ccc(-c3ccccc3)c(-c3ccccn3)n2)cc1</smiles>

3,6-Diphenyl-[2,2']bipyridinyl (8d)<smiles>Ic1ccccc1-c1cc(-c2ccccc2)cc(-c2ccccn2)n1</smiles>

\section{4,6-Diphenyl-[2,2']bipyridinyl $(9 \mathrm{~d}=8 \mathrm{~g})$}

Procedure A:

A mixture of 5-phenyl-3-pyridin-2-yl-[1,2,4]triazine $(0.023 \mathrm{~g}, 0.1 \mathrm{mmol})$, pyrrolidine $(0.018 \mathrm{ml}, 0.2$ $\mathrm{mmol})$ and acetophenone $(0.024 \mathrm{~g}, 0.2 \mathrm{mmol})$ was irradiated at $150^{\circ} \mathrm{C}$ for $60 \mathrm{~min}$. to give the mixture of 8d and 9d (0.022 g, 71\% combined yield, 6:1, 8d:9d from 'H NMR) which was separated by flash chromatography on silica gel (petroleum ether:ethyl acetate, 5:1).

\section{Procedure A:}

A mixture of 5-phenyl-3-pyridin-2-yl-[1,2,4]triazine $(0.023 \mathrm{~g}, 0.1 \mathrm{mmol})$, pyrrolidine $(0.018 \mathrm{ml}, 0.2$ mmol $)$ and phenylacetaldehyde $(0.023 \mathrm{ml}, 0.2 \mathrm{mmol})$ was irradiated at $120^{\circ} \mathrm{C}$ for $15 \mathrm{~min}$. Purification by flash chromatography on silica gel (petroleum ether:ethyl acetate, 5:1) gave the title compound $\mathbf{8 g}$ $(0.026 \mathrm{~g}, 85 \%)$ 
For 8d. Brown solid: $\mathrm{mp} 81-83^{\circ} \mathrm{C}$ (hexane- $\mathrm{CH}_{2} \mathrm{Cl}_{2}$ ); $v_{\max }$ (film) 3058, 2923, 1652, 1582, 1454, 1377, $1090,1023,760,651 \mathrm{~cm}^{-1} ; \delta_{\mathrm{H}}\left(400 \mathrm{MHz}, \mathrm{CDCl}_{3}\right) 8.42(1 \mathrm{H}, \mathrm{ddd}, J=4.8,2.0,0.8 \mathrm{~Hz}, \mathrm{H}-\mathrm{k}), 8.07-8.04$ (2 H, m, H-l,p), 7.79 (2 H, m, H-a,b), 7.59 (1 H, td, $J=8.0,2.0 \mathrm{~Hz}, \mathrm{H}-\mathrm{i}), 7.53$ (1 H, ddd, $J=8.0,1.2$, 0.8 Hz, H-h), 7.44-7.39 (2 H, m, H-m,o), 7.37-7.32 (1 H, m, H-n), 7.21-7.09 (6 H, m, H-c,d,e,f,g,j); $\delta_{\mathrm{C}}$ $\left(100 \mathrm{MHz}, \mathrm{CDCl}_{3}\right) 158.6(\mathrm{C}), 155.7(\mathrm{C}), 155.6(\mathrm{C}), 148.9(\mathrm{CH}), 139.5(\mathrm{C}), 139.4(\mathrm{CH}), 138.9(\mathrm{C})$, $135.9(\mathrm{CH}), 134.9(\mathrm{C}), 129.2(2 \times \mathrm{CH}), 128.9(\mathrm{CH}), 128.7(2 \times \mathrm{CH}), 128.1(2 \times \mathrm{CH}), 127.1(2 \times \mathrm{CH})$, $126.9(\mathrm{CH}), 124.9(\mathrm{CH}), 122.5(\mathrm{CH}), 119.8(\mathrm{CH}) ; \mathrm{m} / \mathrm{z}(\mathrm{EI}) 308\left(30 \%, \mathrm{M}^{+}\right), 307\left(100 \%, \mathrm{M}^{+}-1\right)$ [HRMS (EI) calcd. for $\mathrm{C}_{22} \mathrm{H}_{15} \mathrm{~N}_{2} 307.1235\left(\mathrm{M}^{+}-1\right)$. Found 307.1235 (-0.1 ppm error)].

For 9d (=8g). White solid: mp 145-146 ${ }^{\circ} \mathrm{C}$ (hexane- $\left.\mathrm{CHCl}_{3}\right) ; v_{\max }$ (film) 2924, 2852, 1583, 1393, 1077, $759 \mathrm{~cm}^{-1} ; \delta_{\mathrm{H}}\left(400 \mathrm{MHz}, \mathrm{CDCl}_{3}\right) 8.72(1 \mathrm{H}, \mathrm{ddd}, J=4.8,2.0,0.8 \mathrm{~Hz}, \mathrm{H}-\mathrm{k}), 8.70(1 \mathrm{H}, \mathrm{ddd}, J=8.0,1.2$, $0.8 \mathrm{~Hz}, \mathrm{H}-\mathrm{h}), 8.66(1 \mathrm{H}, \mathrm{d}, J=1.6 \mathrm{~Hz}, \mathrm{H}-\mathrm{g}), 8.24-8.20(2 \mathrm{H}, \mathrm{m}, \mathrm{H}-1, \mathrm{p}), 8.00(1 \mathrm{H}, \mathrm{d}, J=1.6 \mathrm{~Hz}, \mathrm{H}-\mathrm{a})$, 7.88 (1 H, ddd, $J=8.0,7.6,2.0 \mathrm{~Hz}, \mathrm{H}-\mathrm{i}), 7.85-7.82$ (2 H, m, H-b,f), 7.57-7.51 (4 H, m, H-c,e,m,o), 7.49-7.44 (2 H, m, H-d,n), $7.35(1 \mathrm{H}, \mathrm{ddd}, J=7.6,4.8,1.2 \mathrm{~Hz}, \mathrm{H}-\mathrm{j}) ; \delta_{\mathrm{C}}\left(100 \mathrm{MHz}, \mathrm{CDCl}_{3}\right) 157.11(\mathrm{C})$, $156.3(\mathrm{C}), 156.2(\mathrm{C}), 150.3(\mathrm{C}), 149.0(\mathrm{CH}), 139.4(\mathrm{C}), 138.7(\mathrm{C}), 136.9(\mathrm{CH}), 129.1(\mathrm{CH}), 128.9(2 \times$ $\mathrm{CH}), 128.7(2 \times \mathrm{CH}), 127.2(2 \times \mathrm{CH}), 127.1(3 \times \mathrm{CH}), 123.8(\mathrm{CH}), 121.5(\mathrm{CH}), 118.5(\mathrm{CH}), 117.5$ $(\mathrm{CH}) ; \mathrm{m} / \mathrm{z}(\mathrm{EI}) 308\left(100 \%, \mathrm{M}^{+}\right)$[HRMS (EI) calcd. for $\mathrm{C}_{22} \mathrm{H}_{16} \mathrm{~N}_{2}$ 308.1313. Found 308.1314 (-0.3 ppm error)]. 
<smiles>CCc1c(C)cc(-c2ccccc2)nc1-c1ccccn1</smiles><smiles>CCc1cc(-c2ccccc2)nc(-c2ccccn2)c1C</smiles>

3-Ethyl-4-methyl-6-phenyl-[2,2']bipyridinyl (8e)

\section{4-Ethyl-3-methyl-6-phenyl-[2,2']bipyridinyl (9e)}

\section{Procedure A:}

Irradiation of 5-phenyl-3-pyridin-2-yl-[1,2,4]triazine $(0.023 \mathrm{~g}, 0.1 \mathrm{mmol})$, pyrrolidine $(0.018 \mathrm{ml}, 0.2$ mmol) and 3-pentanone $(0.021 \mathrm{ml}, 0.2 \mathrm{mmol})$ at $120^{\circ} \mathrm{C}$ for $60 \mathrm{~min}$. gave the mixture of $8 \mathbf{e}$ and $9 \mathrm{e}$ ( $0.017 \mathrm{~g}, 60 \%$ combined yield, 2:1, 8e:9e from 'H NMR) which was separated by flash chromatography on silica gel (petroleum ether:ethyl acetate, 5:1).

\section{Procedure B:}

A mixture of 5-phenyl-3-pyridin-2-yl-[1,2,4]triazine $(0.023 \mathrm{~g}, 0.1 \mathrm{mmol}), \mathrm{N}$-methylethylenediamine $(0.026 \mathrm{ml}, 0.30 \mathrm{mmol}), 3$-pentanone $(0.063 \mathrm{ml}, 0.6 \mathrm{mmol})$ and powdered 4A molecular sieves $(0.100 \mathrm{~g})$ in toluene $(1.0 \mathrm{ml})$ was heated at $120^{\circ} \mathrm{C}$ for $46 \mathrm{~h}$. Purification by flash chromatography on silica gel (petroleum ether:ethyl acetate, 5:1) gave the title compound $\mathbf{8 e}(0.016 \mathrm{~g}, 59 \%)$.

For 8e. Colorless oil. $R_{\mathrm{f}} 0.32$ (petroleum ether:ethyl acetate, 5:1); $v_{\max }$ (film) 3061, 2966, 2926, 1584, $1455,1376,1083,760,694 \mathrm{~cm}^{-1} ; \delta_{\mathrm{H}}\left(400 \mathrm{MHz}, \mathrm{CDCl}_{3}\right) 8.66(1 \mathrm{H}, \mathrm{ddd}, J=4.8,2.0,0.8 \mathrm{~Hz}, \mathrm{H}-\mathrm{h}), 8.03-$ 7.96 (2 H, m, H-i,m), $7.86(1 \mathrm{H}, \mathrm{td}, J=7.6,2.0 \mathrm{~Hz}, \mathrm{H}-\mathrm{f}), 7.80(1 \mathrm{H}, \mathrm{ddd}, J=7.6,1.2,0.8 \mathrm{~Hz}, \mathrm{H}-\mathrm{e})$, 7.57 (1 H, s, H-a), 7.47-7.41 (2 H, m, H-j,l), 7.39-7.35 (1 H, m, H-k), 7.34 (1 H, ddd, J = 7.6, 4.8, 1.2 Hz, H-g), 2.87 (2 H, q, J=7.2 Hz, H-c), 2.48 (3 H, s, H-b), 1.09 (3 H, t, J=7.2 Hz, H-d); $\delta_{\mathrm{C}}(100$ $\left.\mathrm{MHz}, \mathrm{CDCl}_{3}\right) 159.8(\mathrm{C}), 156.3(\mathrm{C}), 153.8(\mathrm{C}), 148.1(\mathrm{CH}), 147.2(\mathrm{C}), 139.4(\mathrm{C}), 136.6(\mathrm{CH}), 135.5$ 
(C), $128.5(2 \times \mathrm{CH}), 128.4(\mathrm{CH}), 126.9(2 \times \mathrm{CH}), 124.5(\mathrm{CH}), 122.5(\mathrm{CH}), 122.1(\mathrm{CH}), 21.7\left(\mathrm{CH}_{2}\right)$, $19.5\left(\mathrm{CH}_{3}\right), 14.3\left(\mathrm{CH}_{3}\right) ; \mathrm{m} / \mathrm{z}(\mathrm{EI}) 274\left(45 \%, \mathrm{M}^{+}\right), 259\left(100 \%, \mathrm{M}^{+}-15\right)$ [HRMS (EI) calcd. for $\mathrm{C}_{19} \mathrm{H}_{17} \mathrm{~N}_{2}$ $273.1391\left(\mathrm{M}^{+}-1\right)$. Found 273.1393 (-0.6 ppm error)].

For 9e. Colorless oil. $R_{\mathrm{f}} 0.18$ (petroleum ether:ethyl acetate, 5:1); $v_{\max }$ (film) 2965, 2923, 2852, 1584, $1564,1472,1375,1205,1147,1060,878,746 \mathrm{~cm}^{-1} ; \delta_{\mathrm{H}}\left(400 \mathrm{MHz}, \mathrm{CDCl}_{3}\right) 8.70(1 \mathrm{H}, \mathrm{ddd}, J=4.8,2.0$, 0.8 Hz, H-h), 8.04-8.01 (2 H, m, H-i,m), 7.87-7.83 (2 H, m, H-e,f), 7.60 (1 H, s, H-a), 7.48-7.42 (2 H, m, H-j,l), 7.40-7.35 (1 H, m, H-k), 7.33 (1 H, ddd, $J=7.2,4.8,1.2 \mathrm{~Hz}, \mathrm{H}-\mathrm{g}), 2.79$ (2 H, q, J= 7.6 Hz, H-b), 2.41 (3 H, s, H-d), 1.33 (3 H, t, $J=7.6 \mathrm{~Hz}, \mathrm{H}-\mathrm{c}) ; \delta_{\mathrm{C}}\left(100 \mathrm{MHz}, \mathrm{CDCl}_{3}\right) 158.6$ (C), 156.7 (C), $154.4(\mathrm{C}), 153.5(\mathrm{C}), 148.2(\mathrm{CH}), 139.7(\mathrm{C}), 137.1(\mathrm{CH}), 136.3(\mathrm{CH}), 128.9(\mathrm{C}), 128.6(2 \times \mathrm{CH}), 128.5$ $(\mathrm{CH}), 126.9(2 \times \mathrm{CH}), 122.6(\mathrm{CH}), 119.7(\mathrm{CH}), 26.4\left(\mathrm{CH}_{2}\right), 14.9\left(\mathrm{CH}_{3}\right), 13.7\left(\mathrm{CH}_{3}\right) ; \mathrm{m} / \mathrm{z}(\mathrm{EI}) 274$ $\left(65 \%, \mathrm{M}^{+}\right), 273\left(100 \%, \mathrm{M}^{+}-1\right)$ [HRMS (EI) calcd. for $\mathrm{C}_{19} \mathrm{H}_{17} \mathrm{~N}_{2} 273.1391\left(\mathrm{M}^{+}-1\right)$. Found $273.1391(0.0$ ppm error)].

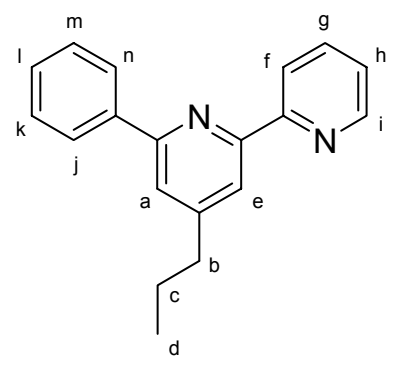

6-Phenyl-4-propyl-[2,2']bipyridinyl (8f)

\section{Procedure A:}

A mixture of 5-phenyl-3-pyridin-2-yl-[1,2,4]triazine $(0.023 \mathrm{~g}, 0.1 \mathrm{mmol})$, pyrrolidine $(0.018 \mathrm{ml}, 0.2$ mmol $)$ and pentanal $(0.021 \mathrm{ml}, 0.2 \mathrm{mmol})$ was irradiated at $120^{\circ} \mathrm{C}$ for $30 \mathrm{~min}$. Purification by flash chromatography on silica gel (petroleum ether:ethyl acetate, 5:1) gave the title compound $\mathbf{8 f}(0.022 \mathrm{~g}$, $81 \%$ as a white solid: $\mathrm{mp} 74-75^{\circ} \mathrm{C}$ (hexane- $\mathrm{CHCl}_{3}$ ); $v_{\max }$ (film) 3061, 2926, 2869, 1603, 1584, 1409, 794, $640 \mathrm{~cm}^{-1} ; \delta_{\mathrm{H}}\left(400 \mathrm{MHz}, \mathrm{CDCl}_{3}\right) 8.70(1 \mathrm{H}, \mathrm{ddd}, J=4.8,1.6,0.8 \mathrm{~Hz}, \mathrm{H}-\mathrm{i}), 8.65(1 \mathrm{H}, \mathrm{ddd}, J=8.0$, 
1.2, $0.8 \mathrm{~Hz}, \mathrm{H}-\mathrm{f}), 8.23$ (1 H, d, $J=1.6 \mathrm{~Hz}, \mathrm{H}-\mathrm{e}), 8.17-8.13(2 \mathrm{H}, \mathrm{m}, \mathrm{H}-\mathrm{j}, \mathrm{n}), 7.84$ (1 H, ddd, $J=8.0,7.6$, $1.6 \mathrm{~Hz}, \mathrm{H}-\mathrm{g}), 7.60$ (1 H, d, $J=1.6 \mathrm{~Hz}, \mathrm{H}-\mathrm{a}), 7.53-7.47$ (2 H, m, H-k,m), 7.46-7.40 (1 H, m, H-1), 7.32 (1 H, ddd, $J=7.6,4.8,1.2 \mathrm{~Hz}, \mathrm{H}-\mathrm{h}), 2.75$ (2 H, t, $J=7.6 \mathrm{~Hz}, \mathrm{H}-\mathrm{b}), 1.83-1.73$ (2 H, m, H-c), 1.00 (3 H, t, $J=7.2 \mathrm{~Hz}, \mathrm{H}-\mathrm{d}) ; \delta_{\mathrm{C}}\left(100 \mathrm{MHz}, \mathrm{CDCl}_{3}\right) 156.5(\mathrm{C}), 156.4(\mathrm{C}), 155.5(\mathrm{C}), 153.3(\mathrm{C}), 148.9(\mathrm{CH})$, $139.5(\mathrm{C}), 136.8(\mathrm{CH}), 128.8(\mathrm{CH}), 128.6(2 \times \mathrm{CH}), 126.9(2 \times \mathrm{CH}), 123.6(\mathrm{CH}), 121.4(\mathrm{CH}), 120.7$ (CH), $119.5(\mathrm{CH}), 37.7\left(\mathrm{CH}_{2}\right), 23.7\left(\mathrm{CH}_{2}\right), 13.8\left(\mathrm{CH}_{3}\right) ; \mathrm{m} / \mathrm{z}(\mathrm{EI}) 274\left(35 \%, \mathrm{M}^{+}\right), 246\left(100 \%, \mathrm{M}^{+}-28\right)$ [HRMS (EI) calcd. for $\mathrm{C}_{19} \mathrm{H}_{18} \mathrm{~N}_{2}$ 274.1469. Found 274.1472 (-1.1 ppm error)].

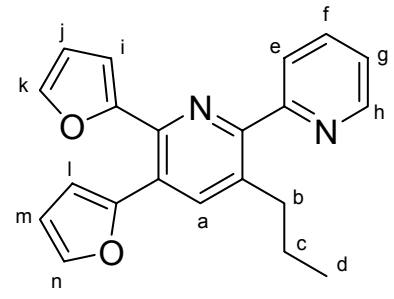

\section{5,6-Difuran-2-yl-3-propyl-[2,2']bipyridinyl (9h)}

\section{Procedure A:}

A mixture of 5,6-difuran-2-yl-3-pyridin-2-yl-[1,2,4]triazine $(0.029 \mathrm{~g}, 0.1 \mathrm{mmol})$, pyrrolidine $(0.018 \mathrm{ml}$, $0.2 \mathrm{mmol})$ and pentanal $(0.021 \mathrm{ml}, 0.2 \mathrm{mmol})$ was irradiated at $120^{\circ} \mathrm{C}$ for $60 \mathrm{~min}$. Purification by flash chromatography on silica gel (petroleum ether:ethyl acetate, $4: 1)$ gave the title compound $\mathbf{9 h}(0.023 \mathrm{~g}$, $69 \%$ ) as a colourless oil: $R_{\mathrm{f}} 0.60$ (petroleum ether:ethyl acetate, 1:1); $v_{\max }$ (film) 2960, 2928, 1588, 1421, 1260, 1089, 1019, $742 \mathrm{~cm}^{-1} ; \delta_{\mathrm{H}}\left(400 \mathrm{MHz}, \mathrm{CDCl}_{3}\right) 8.67(1 \mathrm{H}, \mathrm{ddd}, J=4.8,1.6,0.8 \mathrm{~Hz}, \mathrm{H}-\mathrm{h})$, 7.90-7.86 (2 H, m, H-a,e), $7.82(1 \mathrm{H}, \mathrm{td}, J=7.6,1.6 \mathrm{~Hz}, \mathrm{H}-\mathrm{f}), 7.52(1 \mathrm{H}, \mathrm{dd}, J=2.0,1.2 \mathrm{~Hz}, \mathrm{H}-\mathrm{k}), 7.48$ (1 H, dd, $J=2.0,0.8 \mathrm{~Hz}, \mathrm{H}-\mathrm{n}), 7.30$ (1 H, ddd, $J=7.6,4.8,1.6 \mathrm{~Hz}, \mathrm{H}-\mathrm{g}), 6.51-6.45$ (3 H, m, H-j,1,m), $6.21(1 \mathrm{H}, \mathrm{dd}, J=3.6,0.8 \mathrm{~Hz}, \mathrm{H}-\mathrm{i}), 2.98-2.93(2 \mathrm{H}, \mathrm{m}, \mathrm{H}-\mathrm{b}), 1.62-1.52(2 \mathrm{H}, \mathrm{m}, \mathrm{H}-\mathrm{c}), 0.88(3 \mathrm{H}, \mathrm{t}, J=$ $7.2 \mathrm{~Hz}, \mathrm{H}-\mathrm{d}) ; \delta_{\mathrm{C}}\left(100 \mathrm{MHz}, \mathrm{CDCl}_{3}\right) 158.5(\mathrm{C}), 154.8(\mathrm{C}), 152.5(\mathrm{C}), 150.8(\mathrm{C}), 148.3(\mathrm{CH}), 143.4(\mathrm{C})$, 
$142.6(\mathrm{CH}), 142.5(\mathrm{CH}), 138.8(\mathrm{CH}), 136.6(\mathrm{CH}), 135.8(\mathrm{C}), 124.6(\mathrm{CH}), 124.3(\mathrm{C}), 122.8(\mathrm{CH}), 111.6$ $(\mathrm{CH}), 111.4(\mathrm{CH}), 110.4(\mathrm{CH}), 109.4(\mathrm{CH}), 34.2\left(\mathrm{CH}_{2}\right), 23.9\left(\mathrm{CH}_{2}\right), 13.9\left(\mathrm{CH}_{3}\right) ; \mathrm{m} / \mathrm{z}(\mathrm{EI}) 330(70 \%$, $\left.\mathrm{M}^{+}\right), 315\left(100 \%, \mathrm{M}^{+}-15\right)$ [HRMS (EI) calcd. for $\mathrm{C}_{21} \mathrm{H}_{18} \mathrm{~N}_{2} \mathrm{O}_{2}$ 330.1368. Found 330.1365 (0.9 ppm error)].

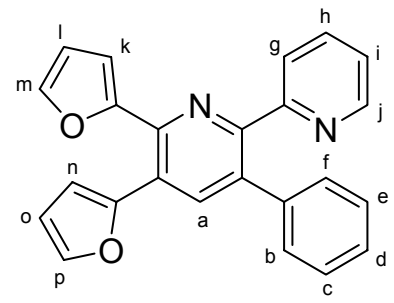

\section{5,6-Difuran-2-yl-3-phenyl-[2,2']bipyridinyl (9i)}

\section{Procedure A:}

A mixture of 5,6-difuran-2-yl-3-pyridin-2-yl-[1,2,4]triazine $(0.029 \mathrm{~g}, 0.1 \mathrm{mmol})$, pyrrolidine $(0.018 \mathrm{ml}$, $0.2 \mathrm{mmol})$ and phenylacetaldehyde $(0.023 \mathrm{ml}, 0.2 \mathrm{mmol})$ was irradiated at $120^{\circ} \mathrm{C}$ for $15 \mathrm{~min}$. Purification by flash chromatography on silica gel (petroleum ether:ethyl acetate, 4:1) gave the title compound $9 \mathbf{i}(0.029 \mathrm{~g}, 82 \%)$ as a yellow solid: $\mathrm{mp} 188-189^{\circ} \mathrm{C}$ (hexane- $\left.\mathrm{CHCl}_{3}\right) ; v_{\max }$ (film) 3056, 2924, 1586, 1416, 1226, 1008, $740 \mathrm{~cm}^{-1} ; \delta_{\mathrm{H}}\left(400 \mathrm{MHz}, \mathrm{CDCl}_{3}\right) 8.45(1 \mathrm{H}, \mathrm{ddd}, J=4.8,1.6,0.8 \mathrm{~Hz}, \mathrm{H}-\mathrm{j}), 7.96$ (1 H, s, H-a), 7.54 (1 H, td, $J=7.6,1.6 \mathrm{~Hz}, \mathrm{H}-\mathrm{h})$, 7.46-7.42 (3 H, m, H-g,m,p), 7.22-7.17 (3 H, m, Hb,d,f), 7.15-7.08 (3 H, m, H-c,e,i), 6.49 (1 H, dd, $J=3.2,0.8 \mathrm{~Hz}, \mathrm{H}-\mathrm{k}), 6.43(1 \mathrm{H}, \mathrm{dd}, J=3.2,1.6 \mathrm{~Hz}$, $\mathrm{H}-\mathrm{o}), 6.41(1 \mathrm{H}, J=3.2,1.6 \mathrm{~Hz}, \mathrm{H}-1), 6.21(1 \mathrm{H}, \mathrm{dd}, J=3.2,0.8 \mathrm{~Hz}, \mathrm{H}-\mathrm{n}) ; \delta_{\mathrm{C}}\left(100 \mathrm{MHz}, \mathrm{CDCl}_{3}\right) 157.6$ (C), $154.6(\mathrm{C}), 152.1(\mathrm{C}), 150.4(\mathrm{C}), 149.1(\mathrm{CH}), 144.9(\mathrm{C}), 142.9(\mathrm{CH}), 142.7(\mathrm{CH}), 139.2(\mathrm{CH})$, $138.9(\mathrm{C}), 135.9(\mathrm{CH}), 135.1(\mathrm{C}), 129.2(2 \times \mathrm{CH}), 128.2(2 \times \mathrm{CH}), 127.2(\mathrm{CH}), 124.9(\mathrm{CH}), 124.2(\mathrm{C})$, $122.6(\mathrm{CH}), 111.7(\mathrm{CH}), 111.6(\mathrm{CH}), 111.3(\mathrm{CH}), 109.7(\mathrm{CH}) ; \mathrm{m} / \mathrm{z}(\mathrm{EI}) 364\left(55 \%, \mathrm{M}^{+}\right), 363(100 \%$, $\mathrm{M}^{+}$-1) [HRMS (EI) calcd. for $\mathrm{C}_{24} \mathrm{H}_{15} \mathrm{~N}_{2} \mathrm{O}_{2} 363.1133\left(\mathrm{M}^{+}-1\right)$. Found 363.1132 (0.2 ppm error)]. 


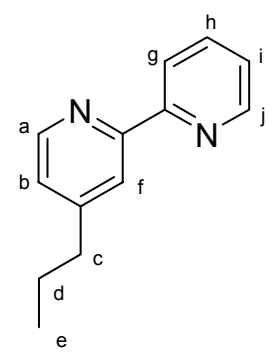

\section{4-Propyl-[2,2']bipyridinyl (9j)}

\section{Procedure A:}

A mixture of 3-pyridin-2-yl-[1,2,4]triazine $(0.015 \mathrm{~g}, 0.1 \mathrm{mmol})$, pyrrolidine $(0.018 \mathrm{ml}, 0.2 \mathrm{mmol})$ and pentanal $(0.021 \mathrm{ml}, 0.2 \mathrm{mmol})$ was irradiated at $150^{\circ} \mathrm{C}$ for $15 \mathrm{~min}$. Purification by flash chromatography on silica gel (petroleum ether:ethyl acetate, 1:1) gave the title compound $\mathbf{9 j}(0.013 \mathrm{~g}$, 67\%) as a colorless oil: $R_{\mathrm{f}} 0.66$ (ethyl acetate); $v_{\max }($ film) $2960,2929,2871,1603,1585,1460,1401$, 793, $744 \mathrm{~cm}^{-1} ; \delta_{\mathrm{H}}\left(400 \mathrm{MHz}, \mathrm{CDCl}_{3}\right) 8.67(1 \mathrm{H}, \mathrm{ddd}, J=4.8,1.6,0.8 \mathrm{~Hz}, \mathrm{H}-\mathrm{j}), 8.55(1 \mathrm{H}, \mathrm{d}, J=4.8 \mathrm{~Hz}$, H-a), $8.38(1 \mathrm{H}$, ddd, $J=7.6,1.2,0.8 \mathrm{~Hz}, \mathrm{H}-\mathrm{g}), 8.23(1 \mathrm{H}, \mathrm{d}, J=1.6 \mathrm{~Hz}, \mathrm{H}-\mathrm{f}), 7.81(1 \mathrm{H}, \mathrm{td}, J=7.6,1.6$ Hz, H-h), 7.30 (1 H, ddd, $J=7.6,4.8,1.2 \mathrm{~Hz}, \mathrm{H}-\mathrm{i}), 7.13$ (1 H, dd, J=4.8, 1.6 Hz, H-b), 2.67 (2 H, t, $J$ $=7.6 \mathrm{~Hz}, \mathrm{H}-\mathrm{c}), 1.77-1.67(2 \mathrm{H}, \mathrm{m}, \mathrm{H}-\mathrm{d}), 0.96(3 \mathrm{H}, \mathrm{t}, J=7.6 \mathrm{~Hz}, \mathrm{H}-\mathrm{e}) ; \delta_{\mathrm{C}}\left(100 \mathrm{MHz}, \mathrm{CDCl}_{3}\right) 156.3$ (C), $155.9(\mathrm{C}), 152.6(\mathrm{C}), 149.1(\mathrm{CH}), 149.0(\mathrm{CH}), 136.9(\mathrm{CH}), 124.0(\mathrm{CH}), 123.6(\mathrm{CH}), 121.2(2 \times$ $\mathrm{CH}), 37.5\left(\mathrm{CH}_{2}\right), 23.5\left(\mathrm{CH}_{2}\right), 13.7\left(\mathrm{CH}_{3}\right) ; \mathrm{m} / \mathrm{z}(\mathrm{EI}) 198\left(25 \%, \mathrm{M}^{+}\right), 170\left(100 \%, \mathrm{M}^{+}-28\right)$ [HRMS (EI) calcd. for $\mathrm{C}_{13} \mathrm{H}_{14} \mathrm{~N}_{2}$ 198.1156. Found 198.1162 (-2.7 ppm error)]. 


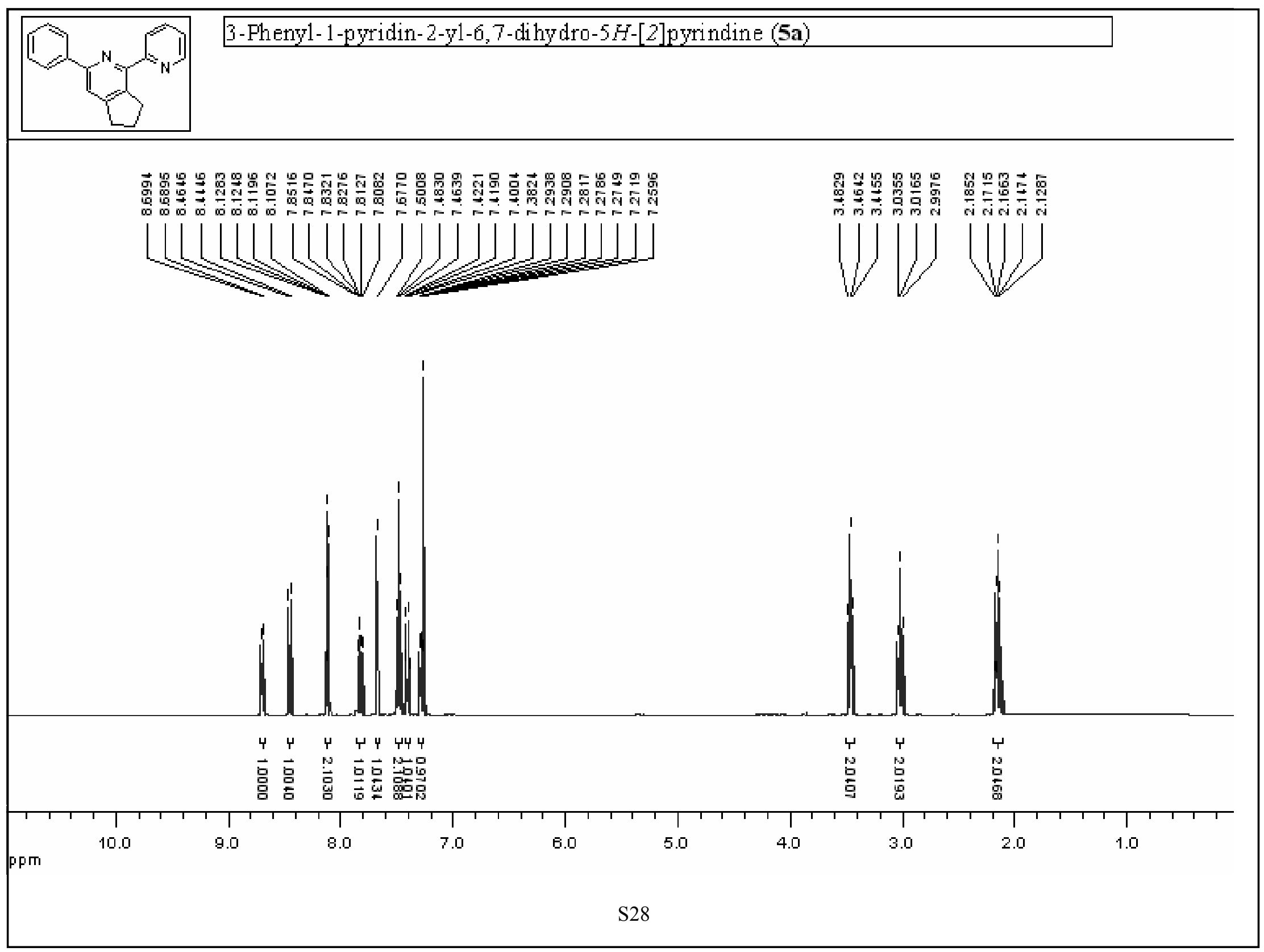




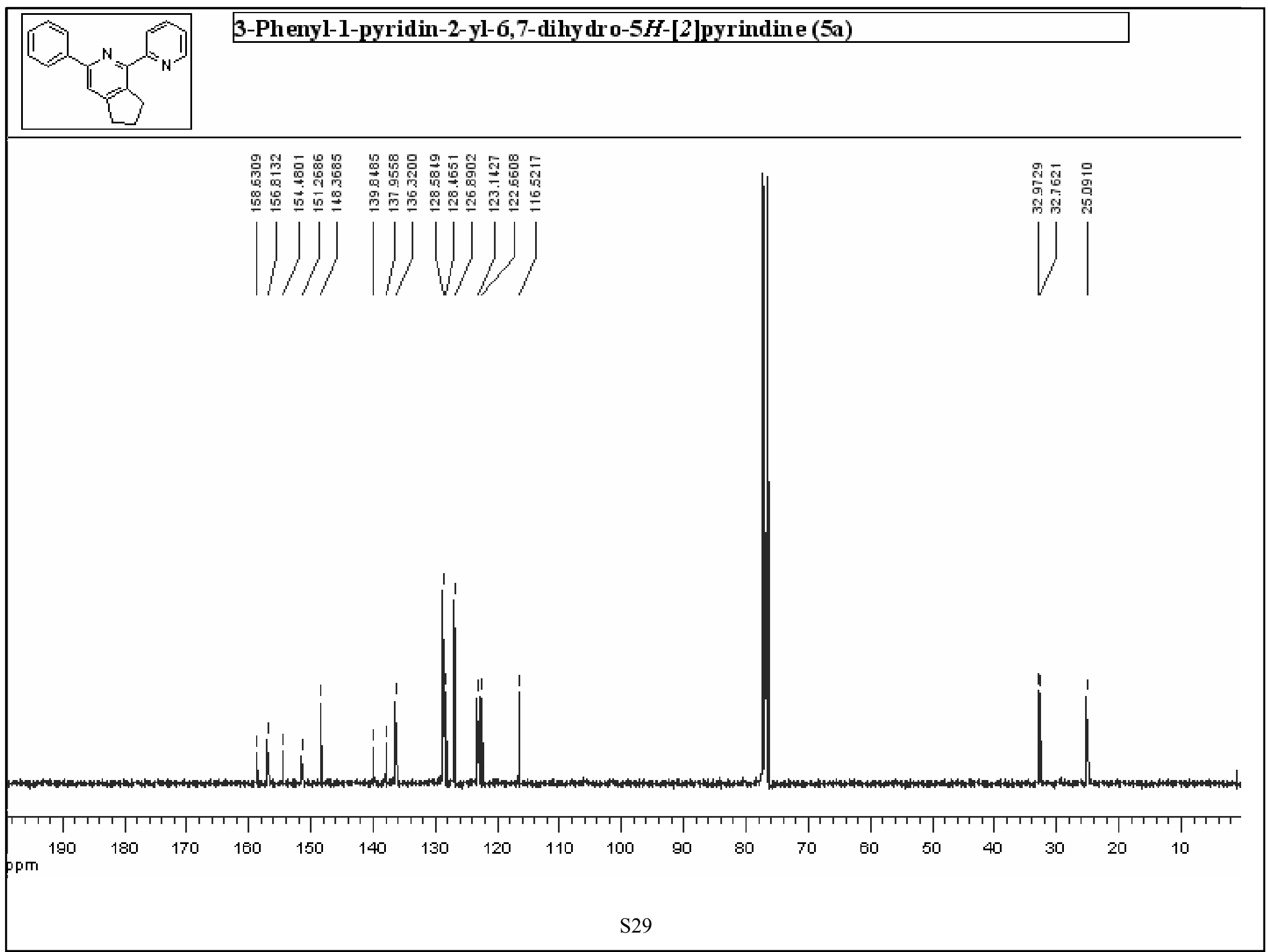



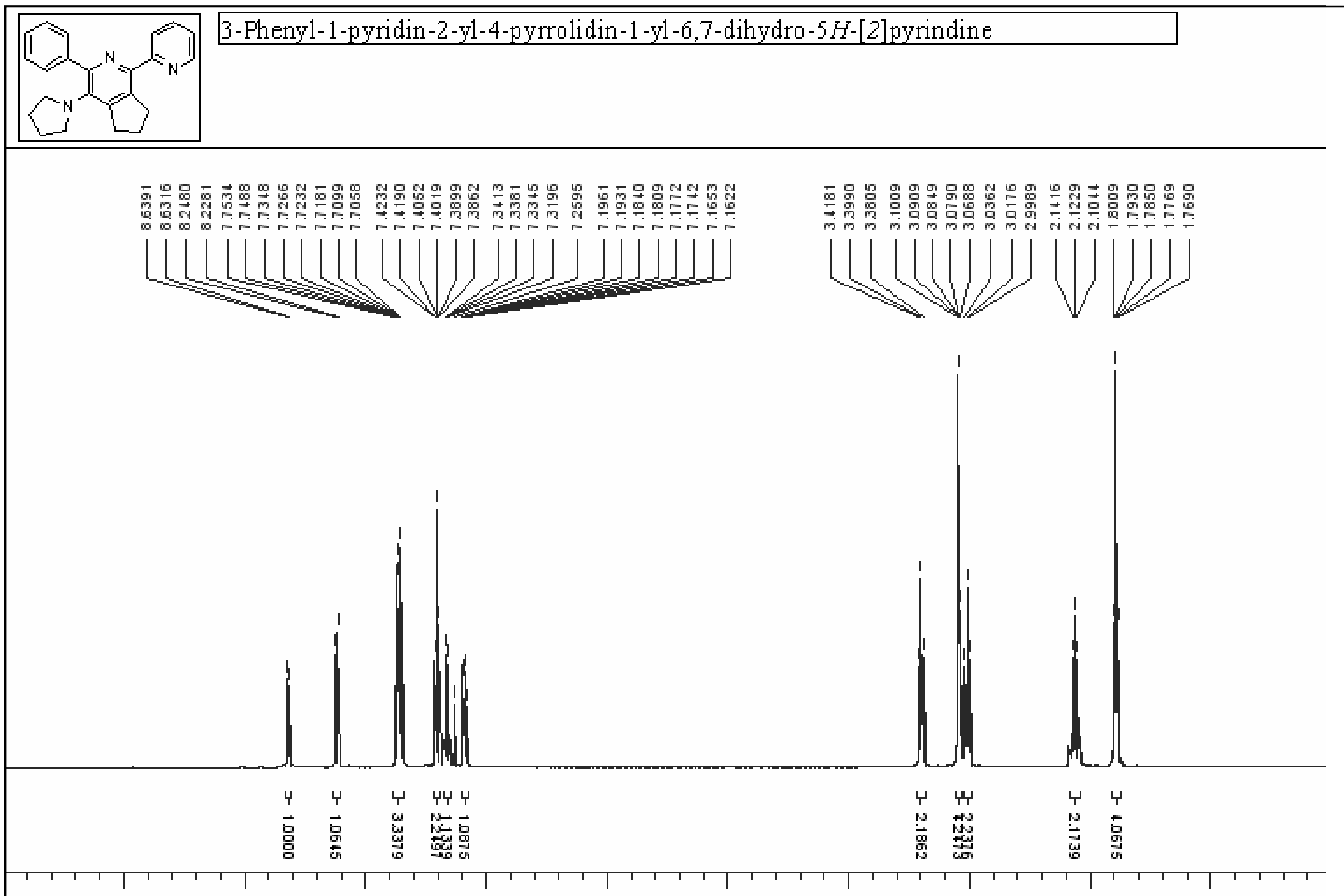

ppm

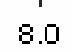

7.0

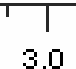

2.0

1.0 


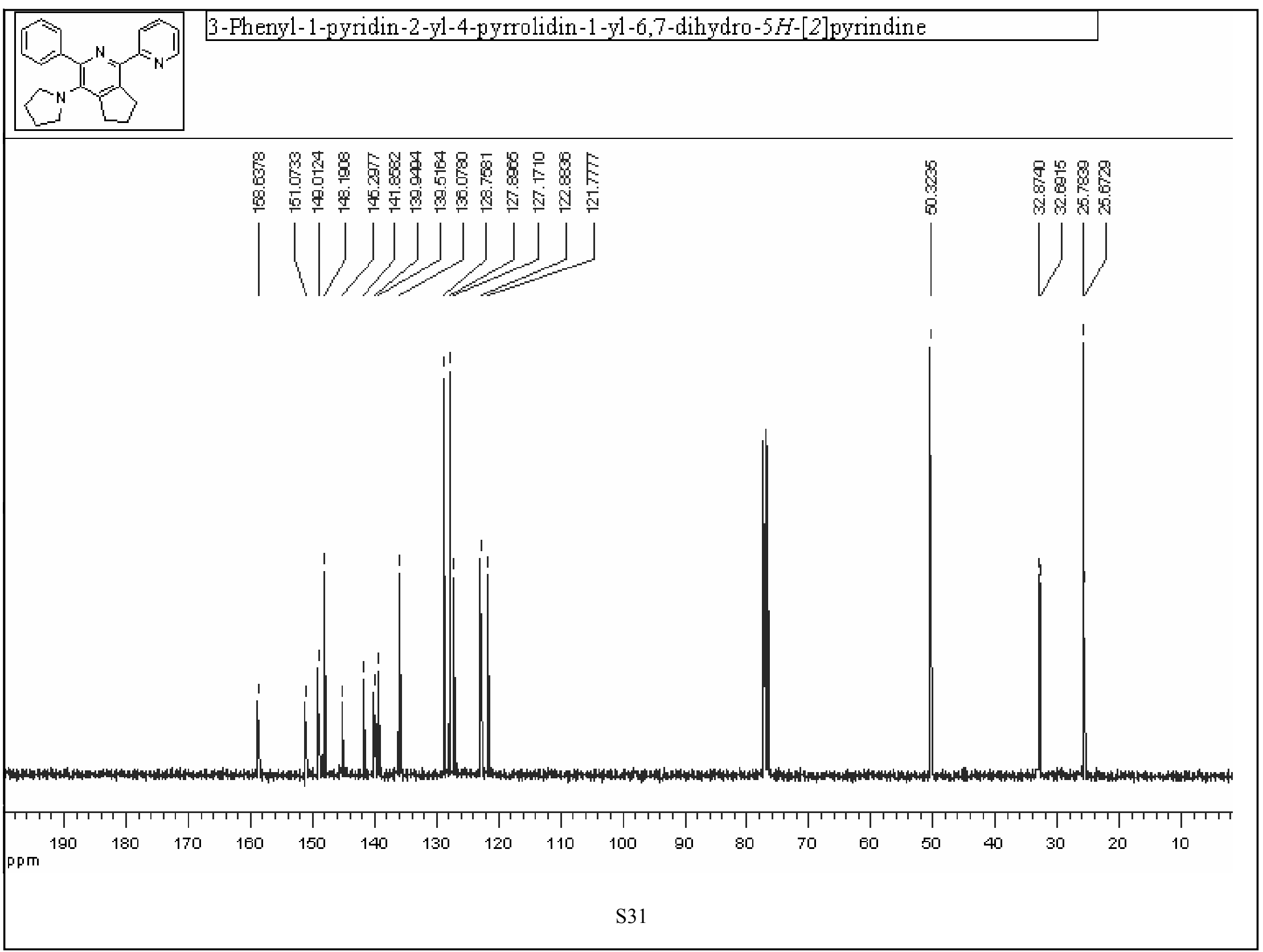




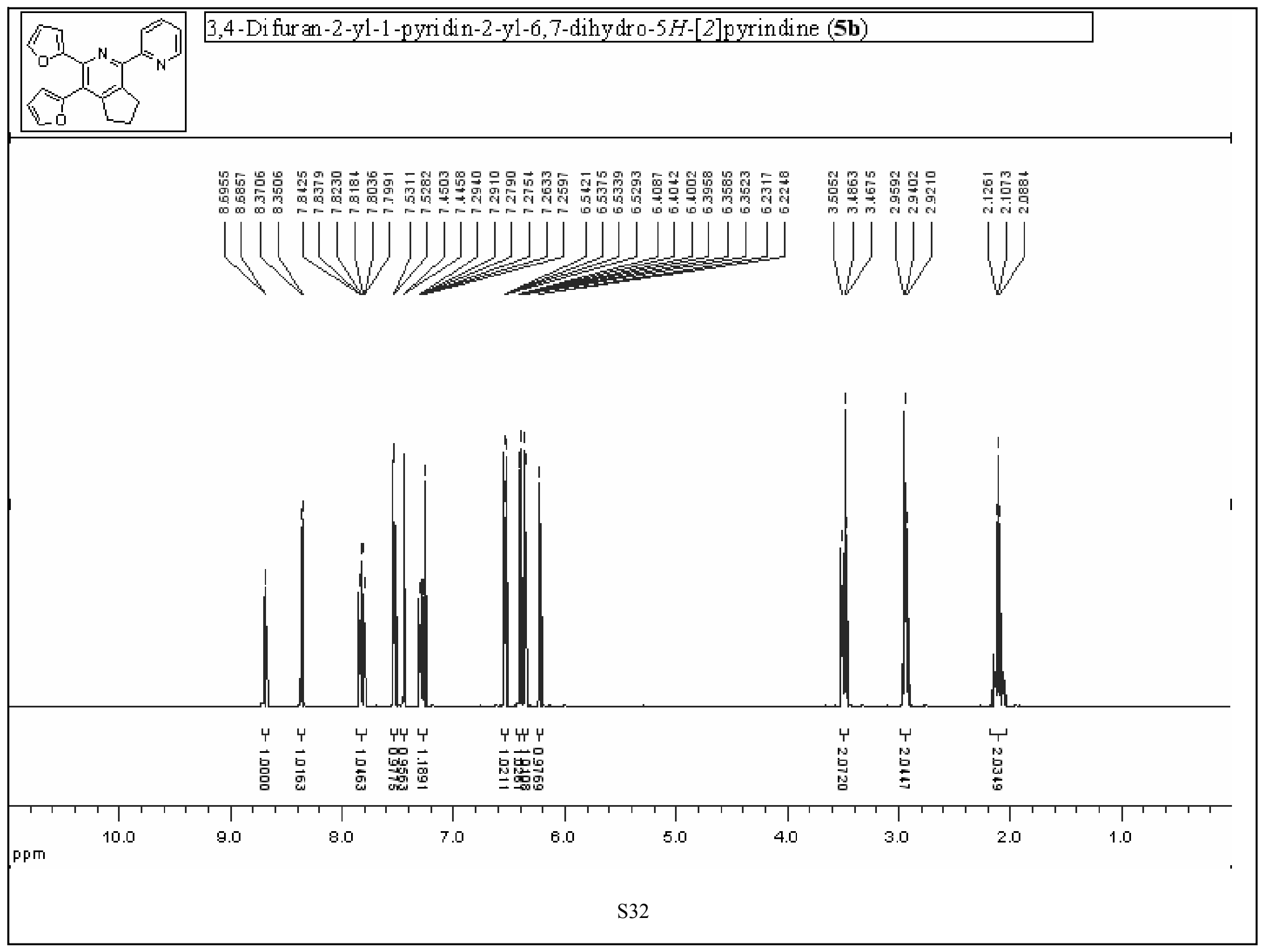




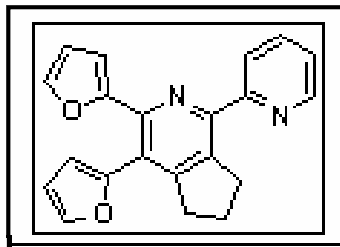

3,4-Difuran-2-yl-1-pyridin-2-yl-6, 7-dihydro-5H-[2]pyrindine (5b)

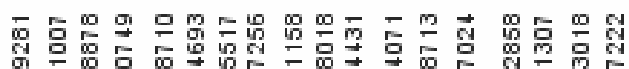

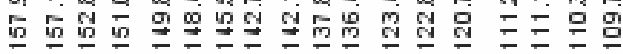

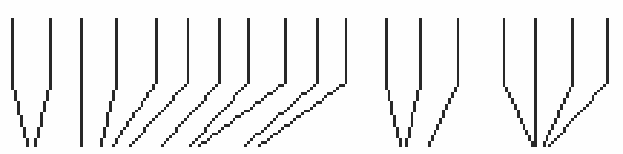

mow

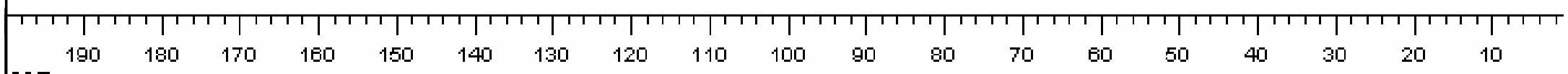

ppm
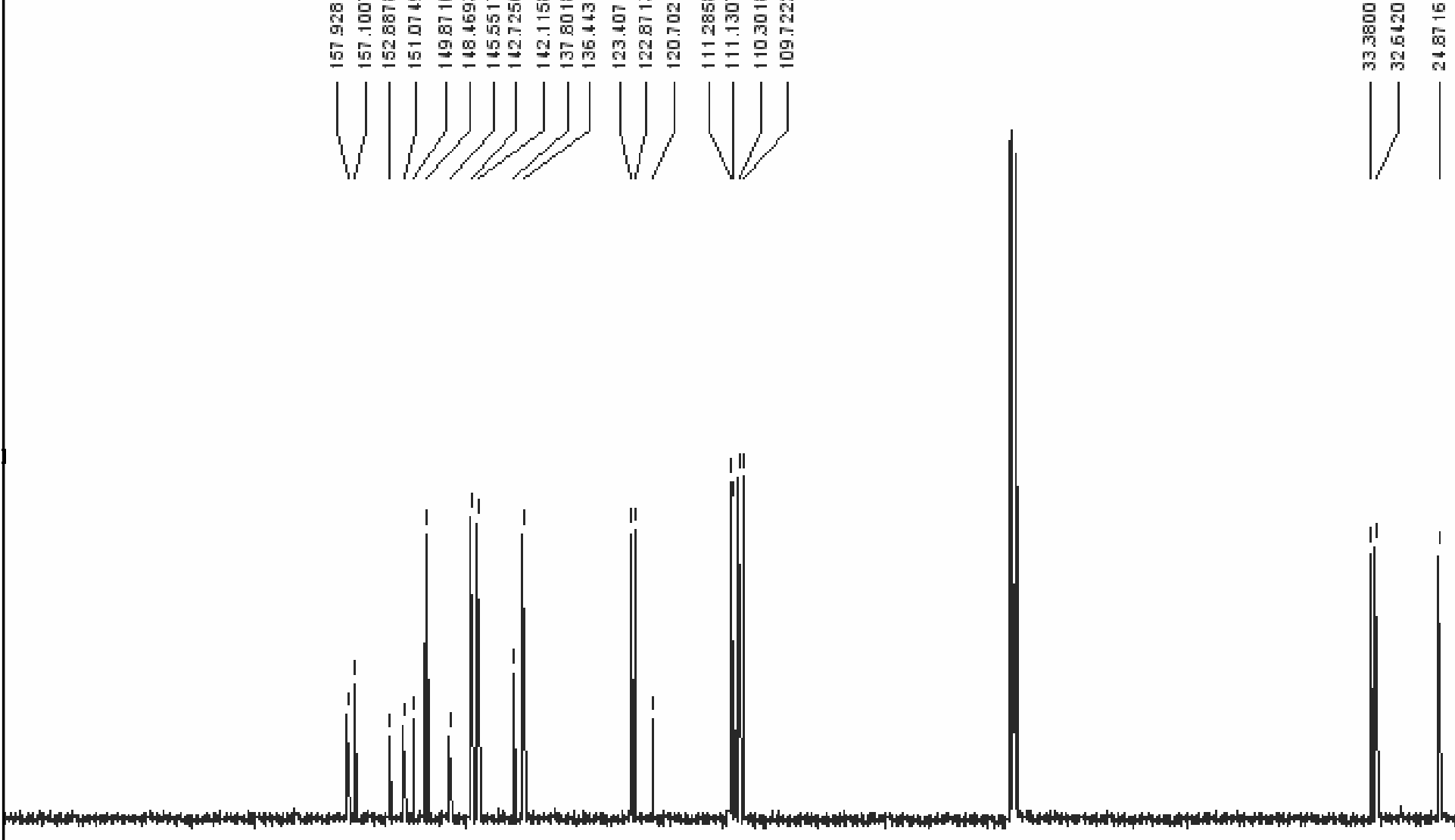


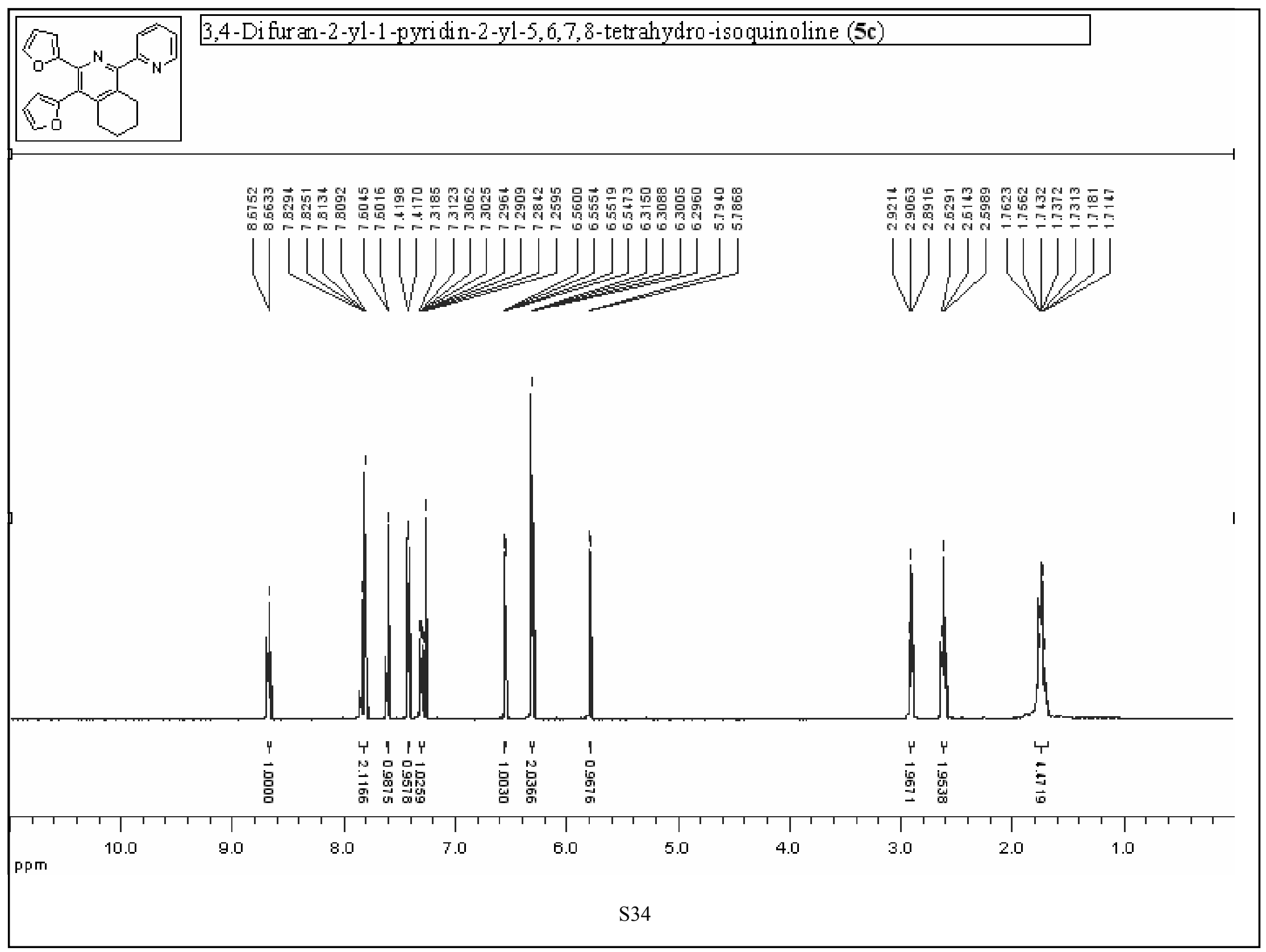




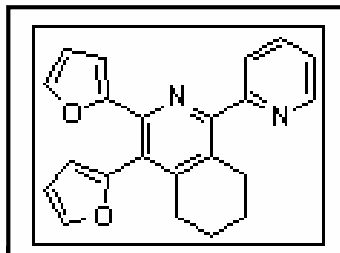

3,4-Difuran-2-yl-1-pyridin-2-yl-5, 6, 7,8-tetrahydro-isoquinoline (5c)

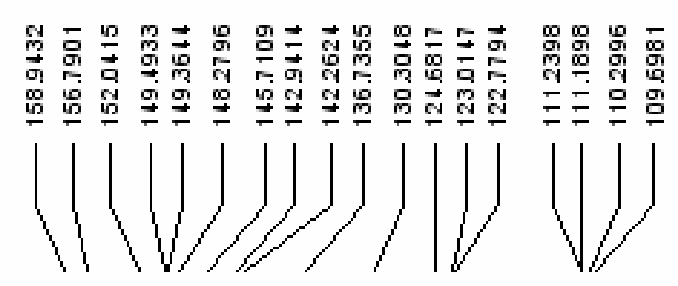

ตั

ले ते लू बू

$1 / 1$
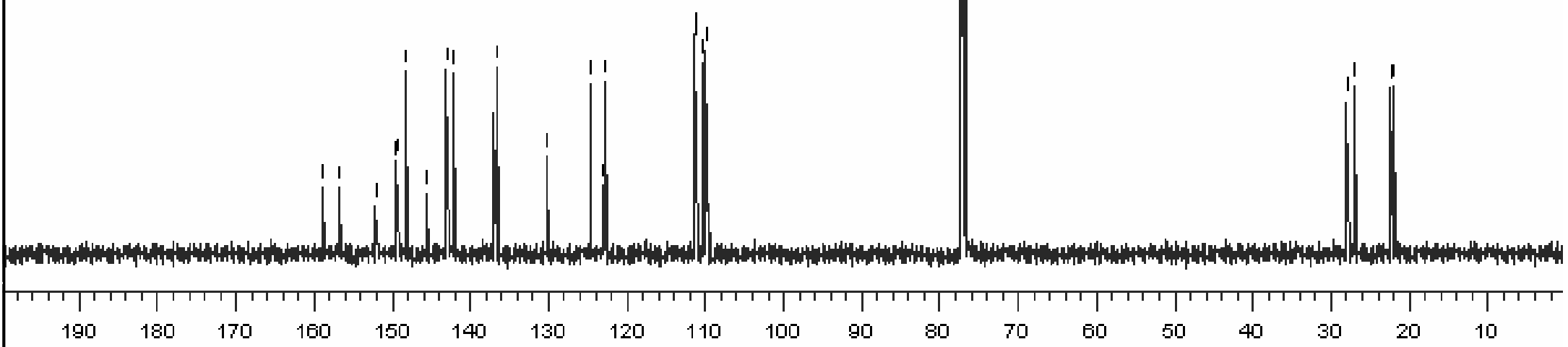

ppm 


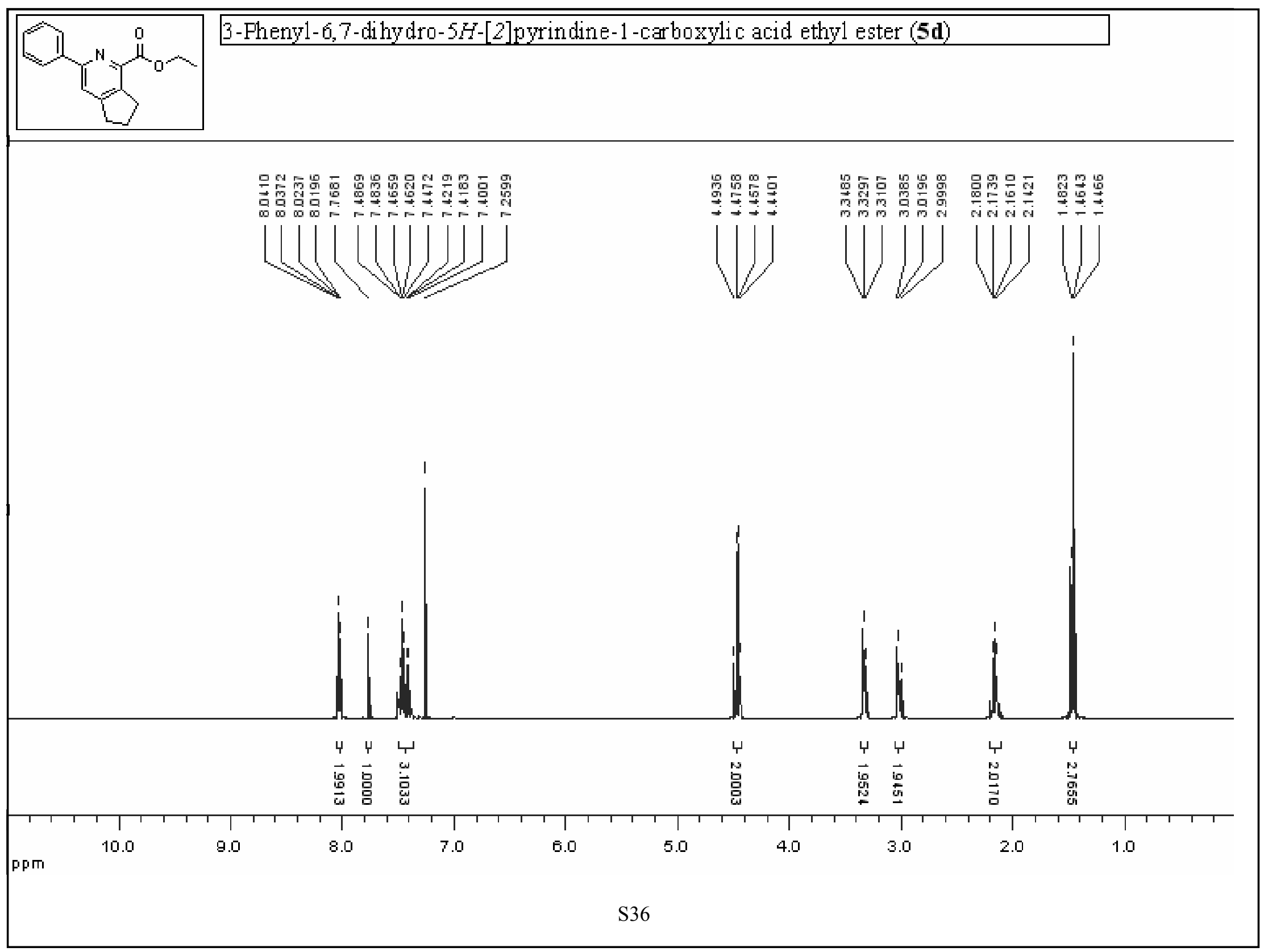




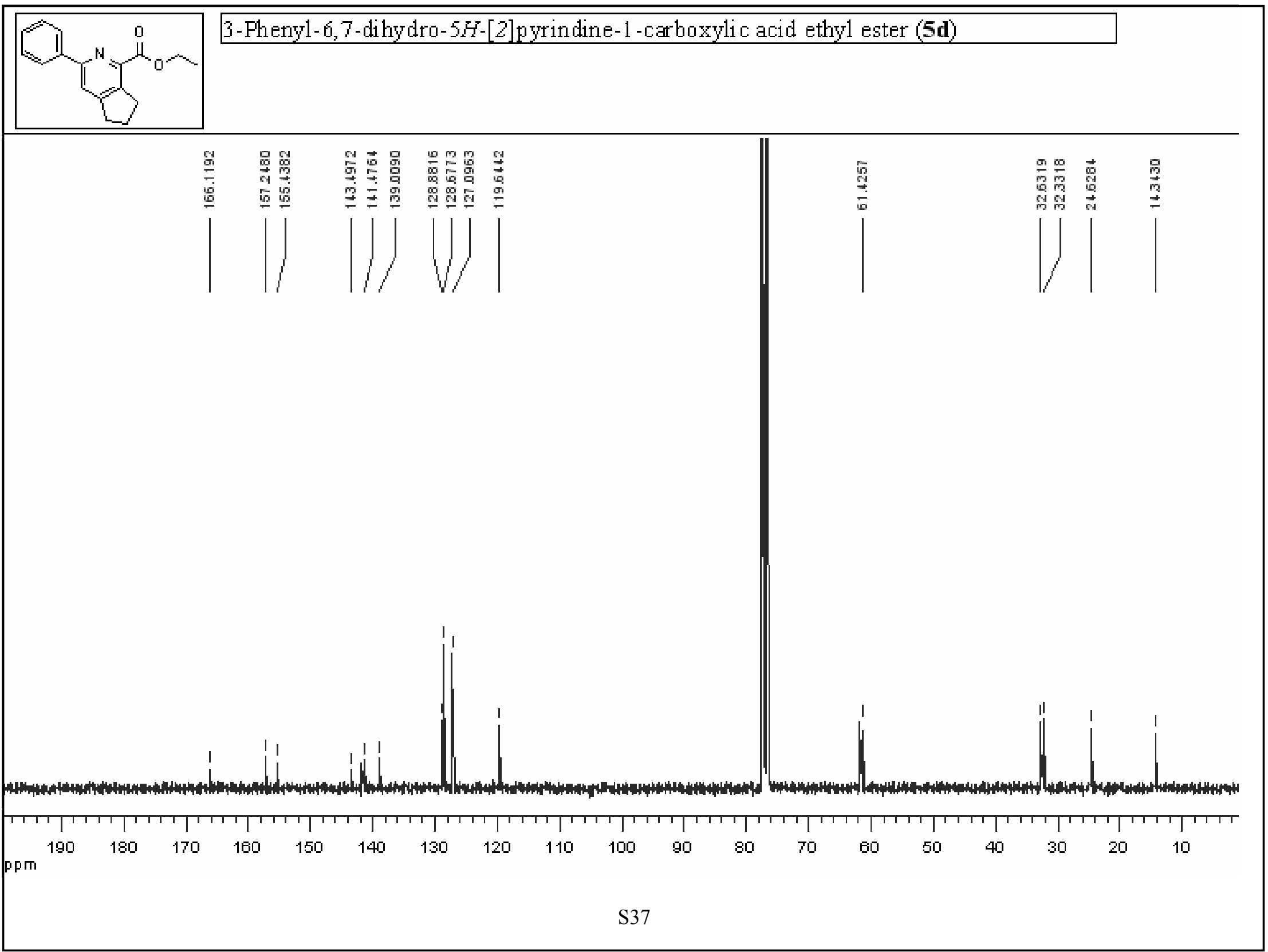




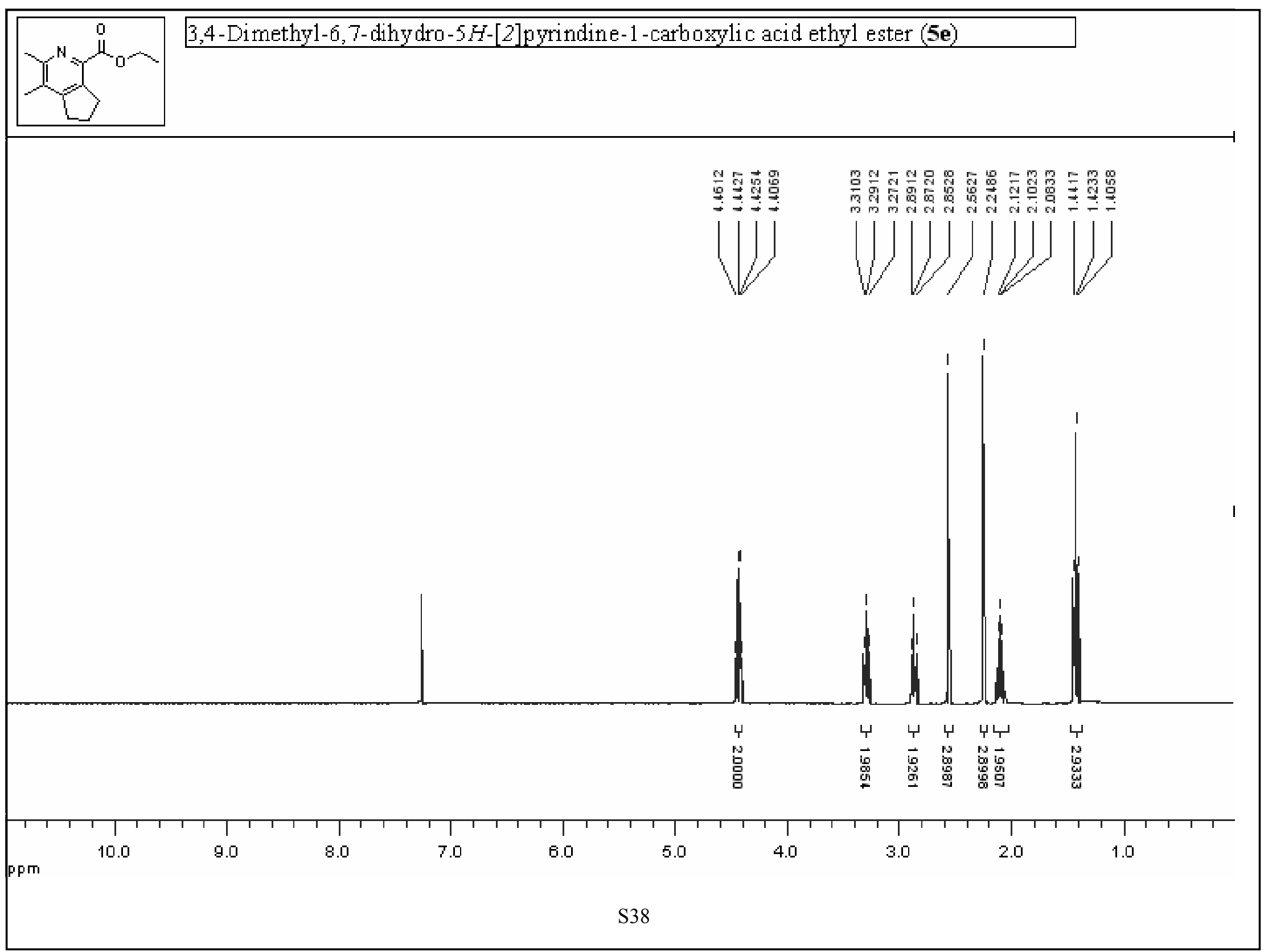




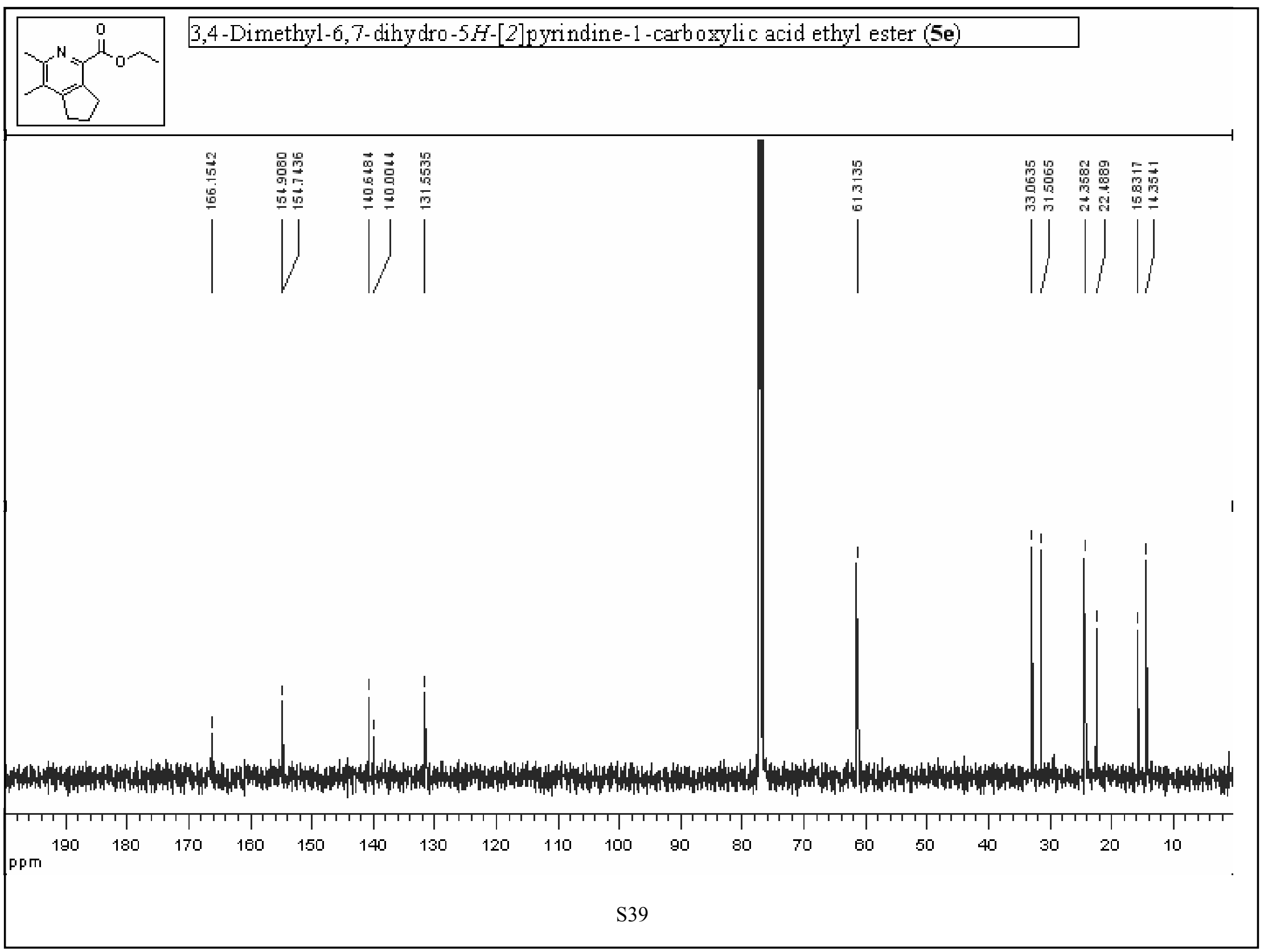



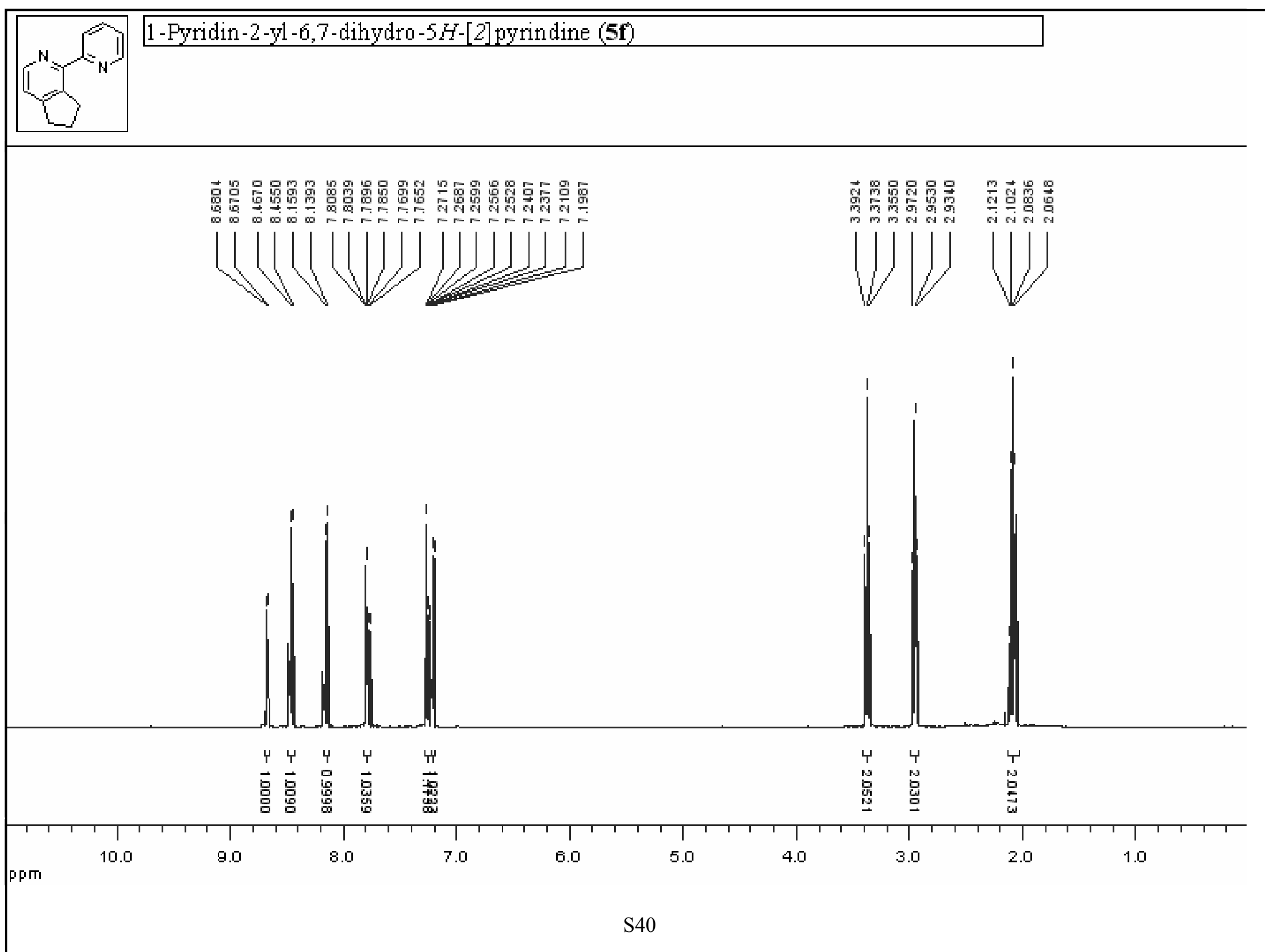


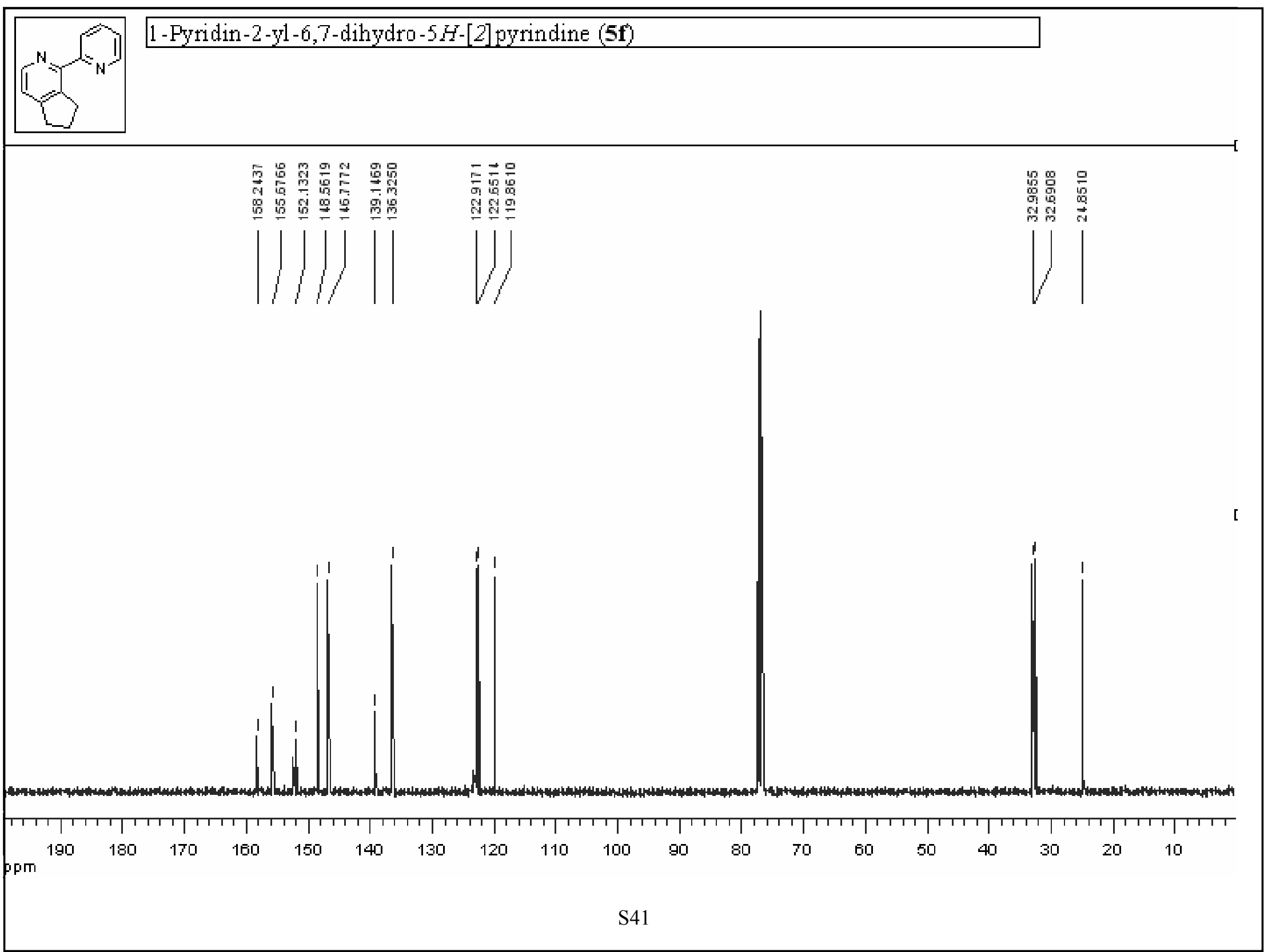




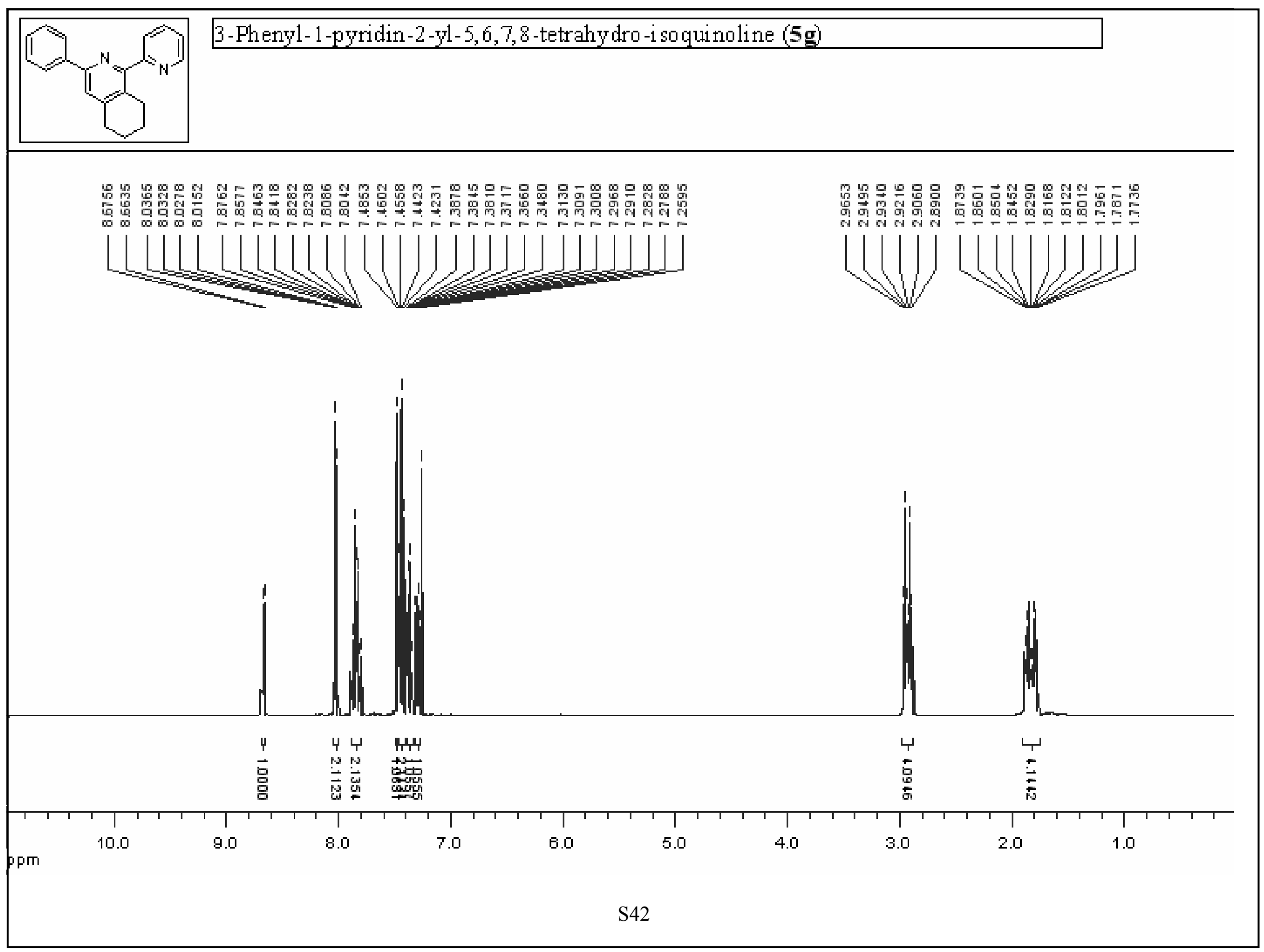




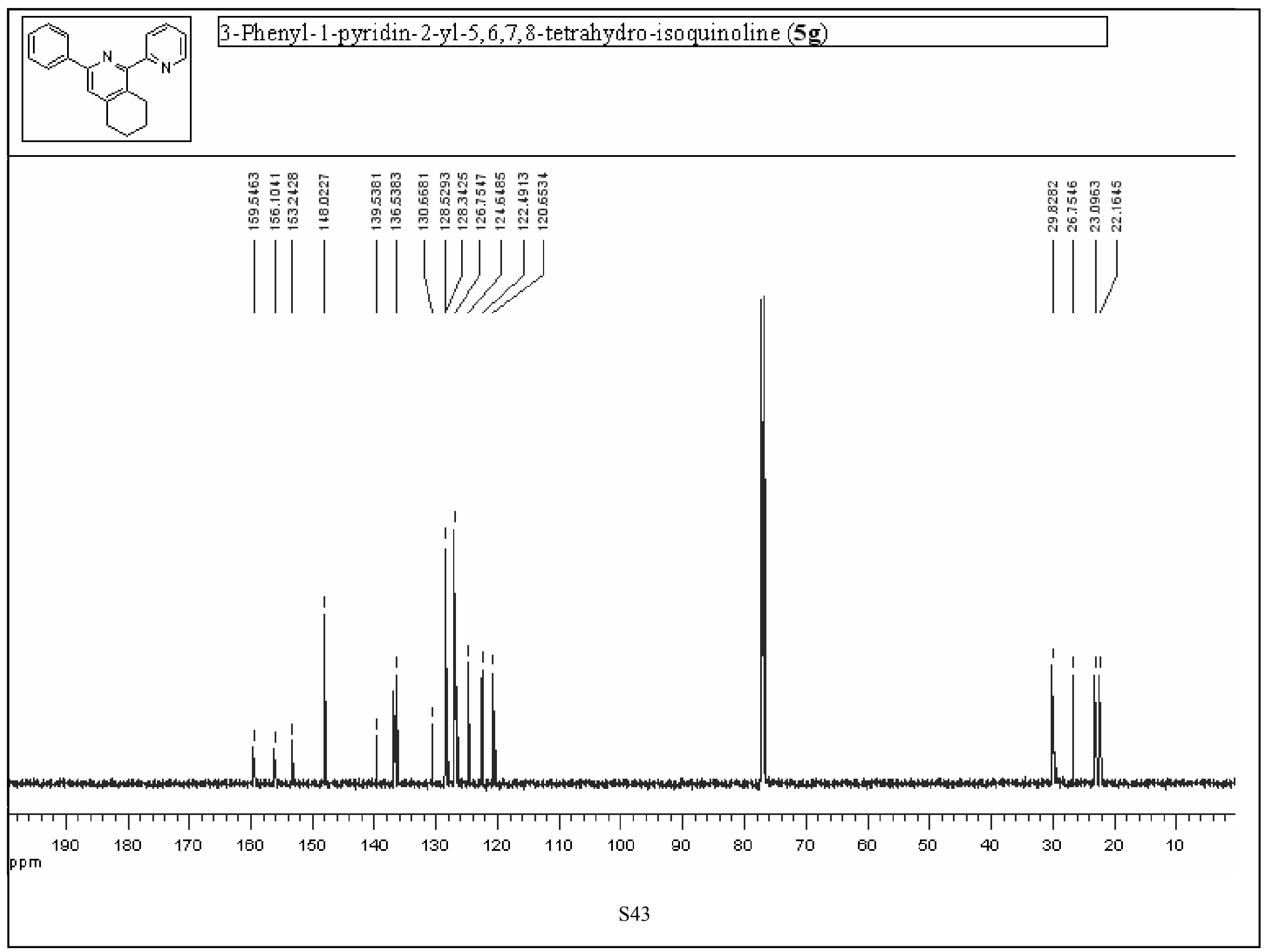




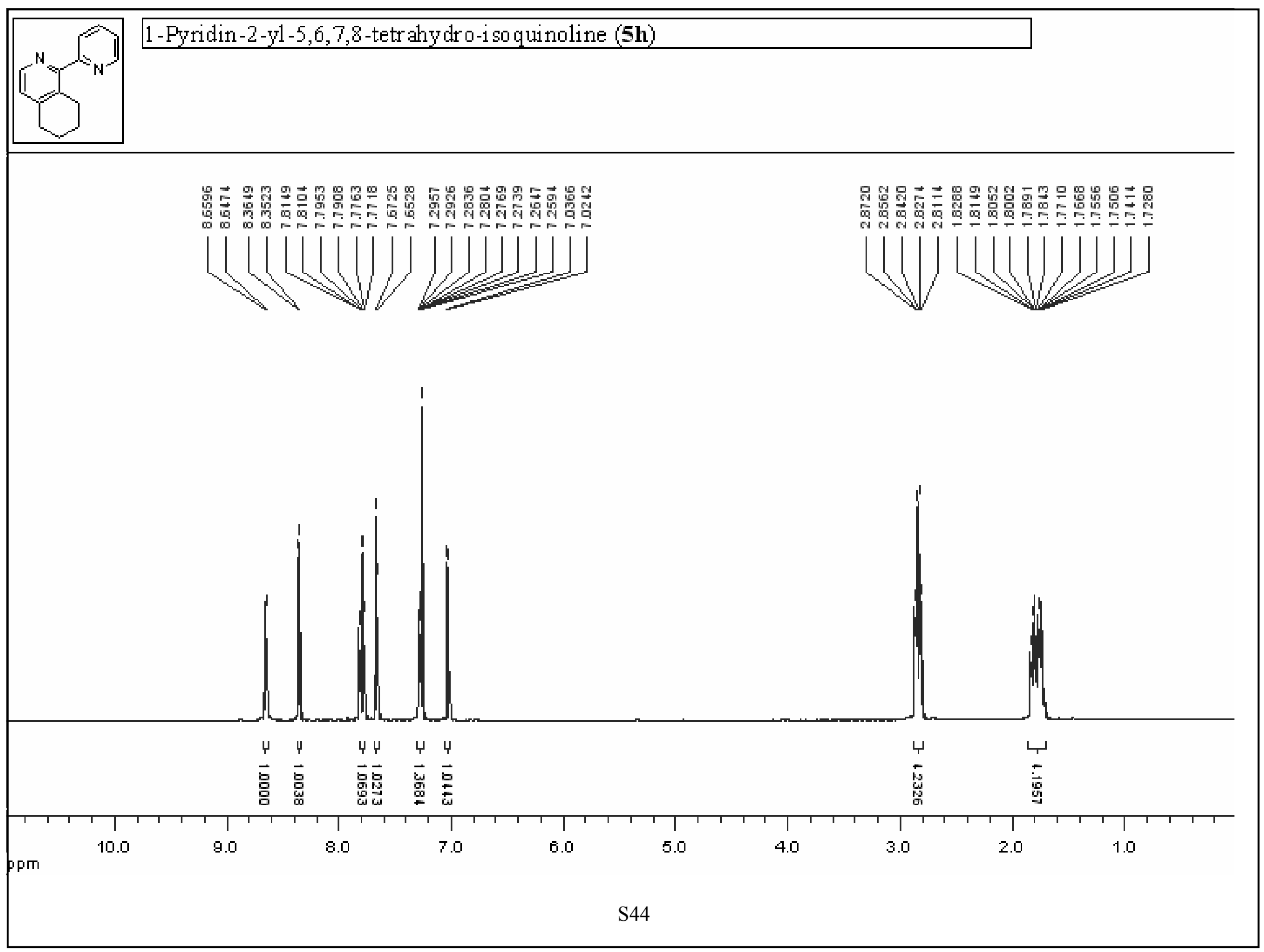




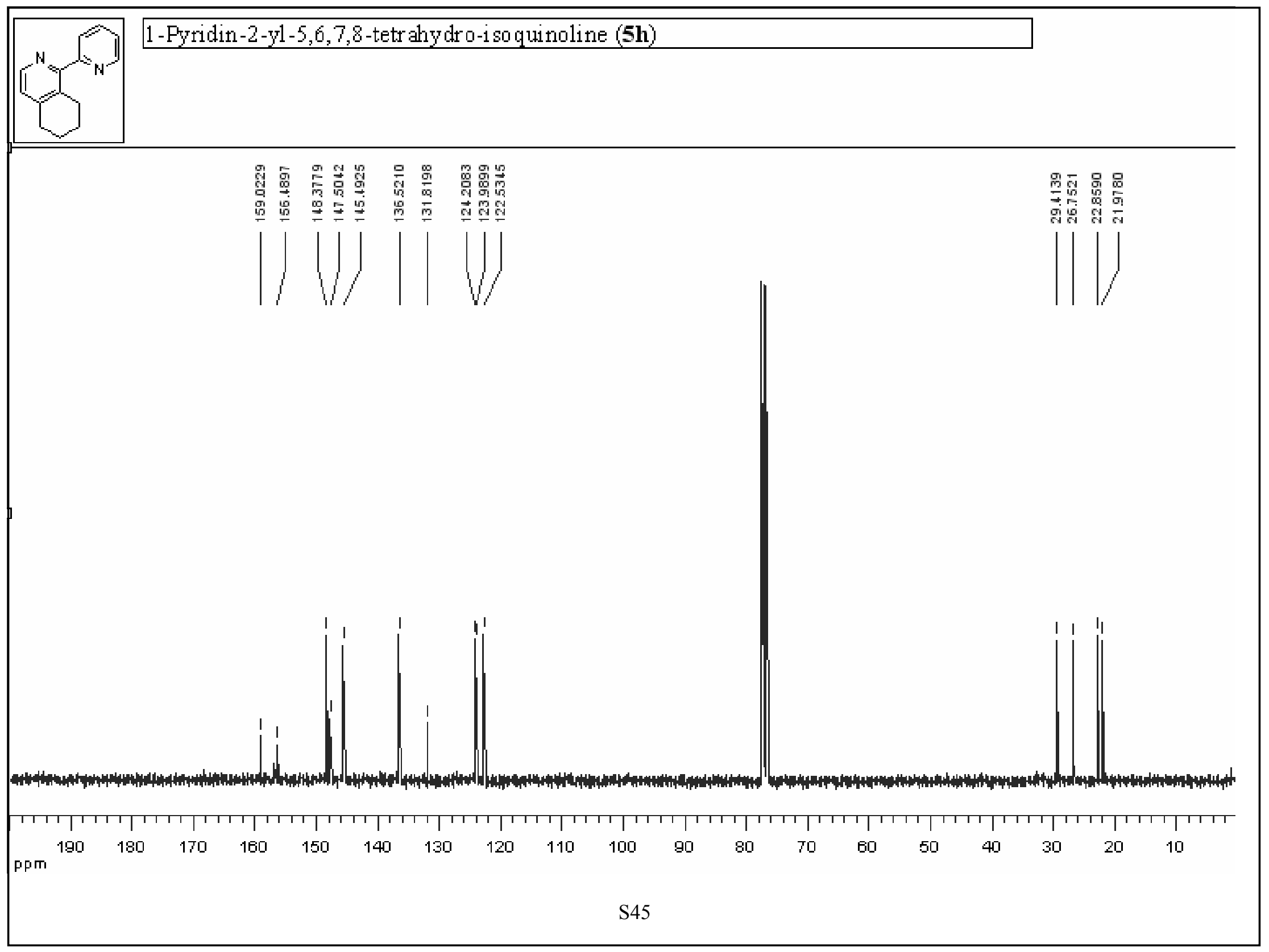




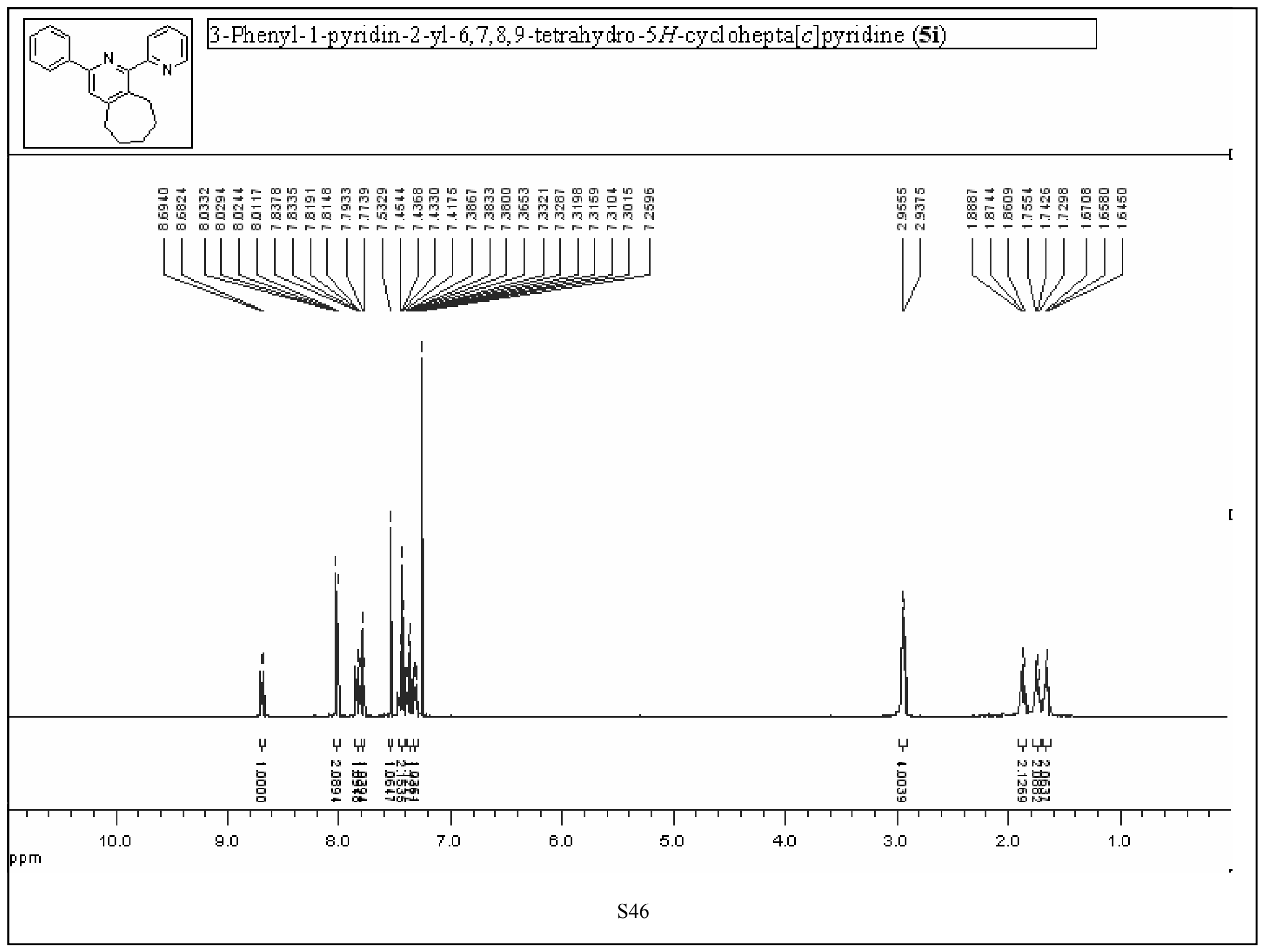



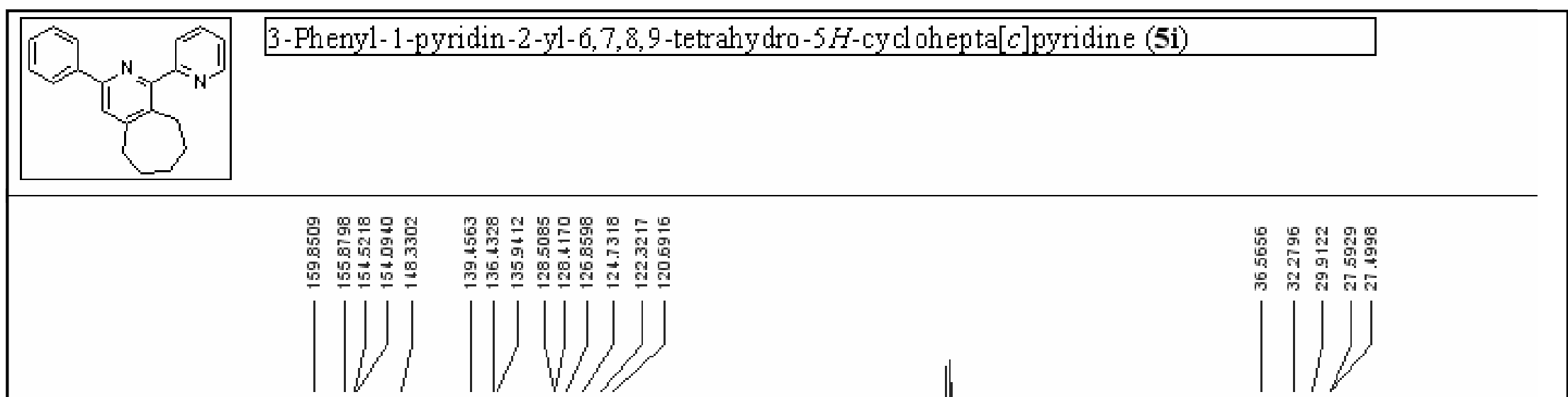

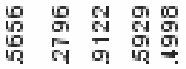

लू लू ते ते

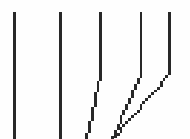

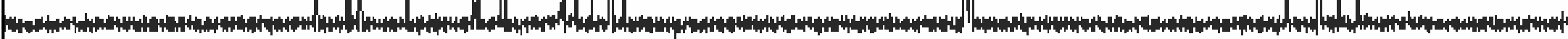

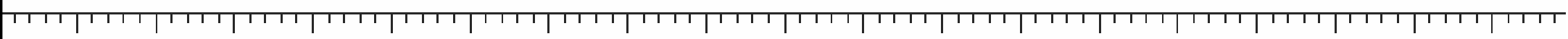
Pp 190

$180 \quad 170$

$160 \quad 150$

$140 \quad 130$

120

100

90

80

70 60




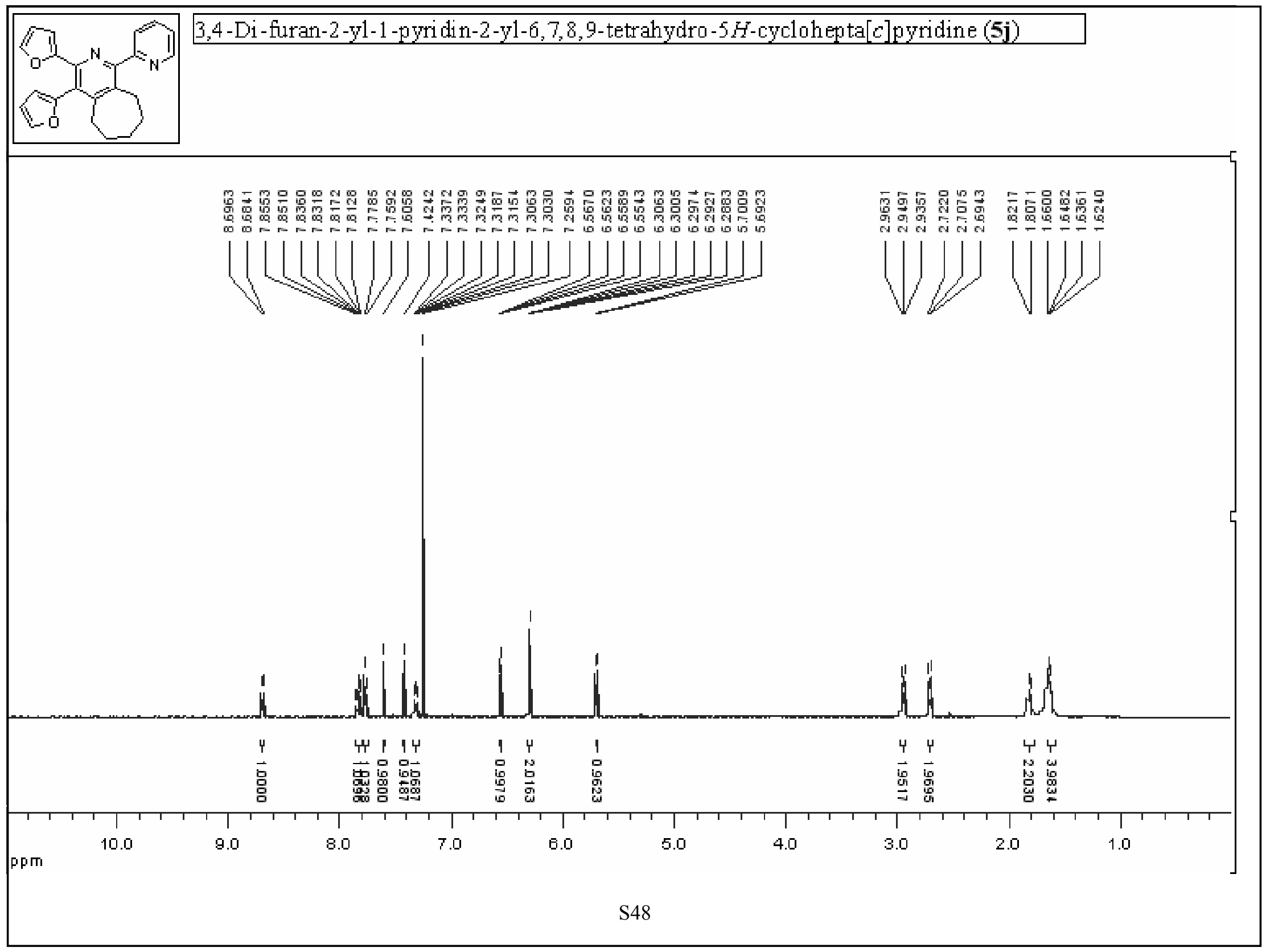




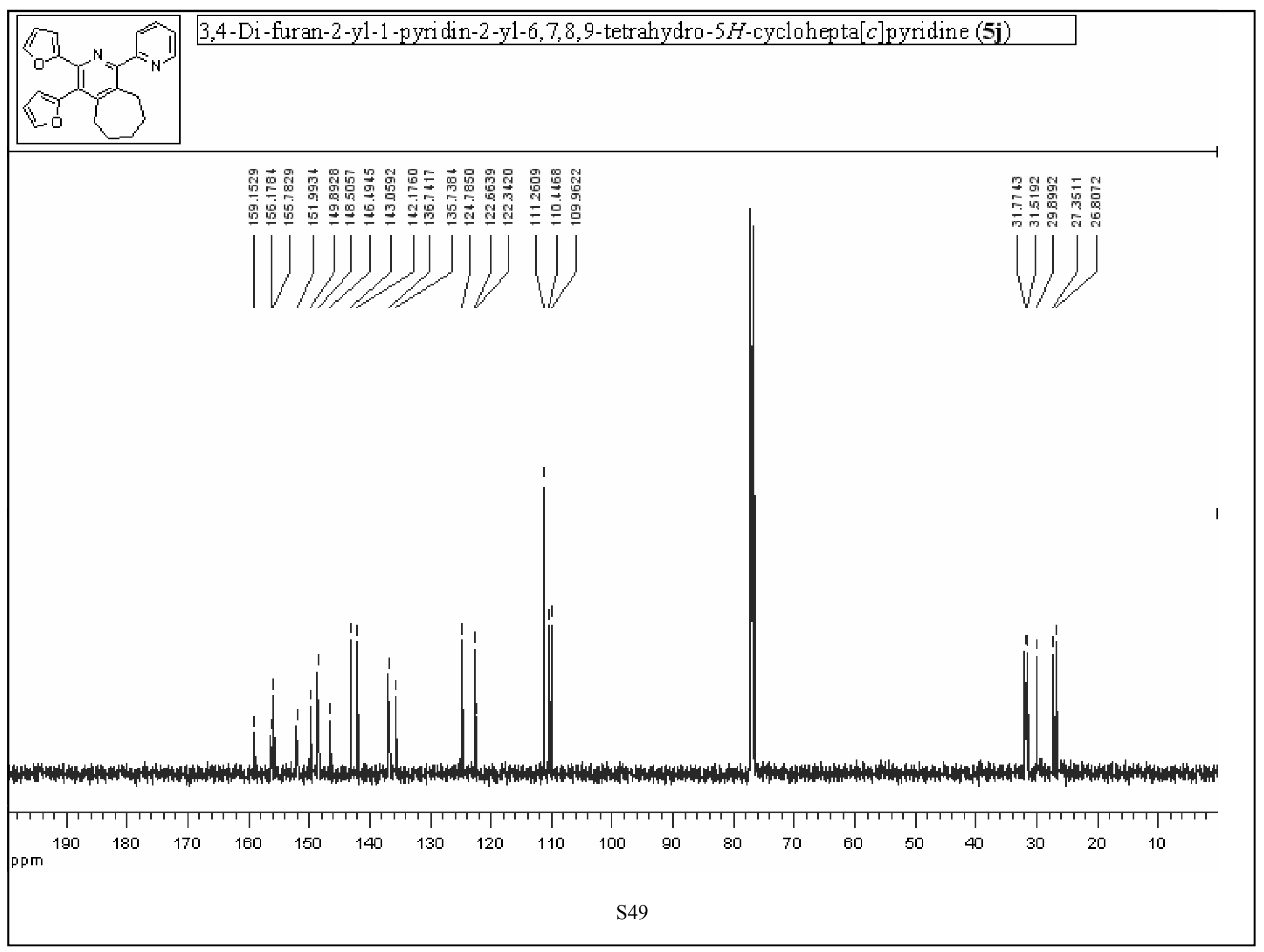




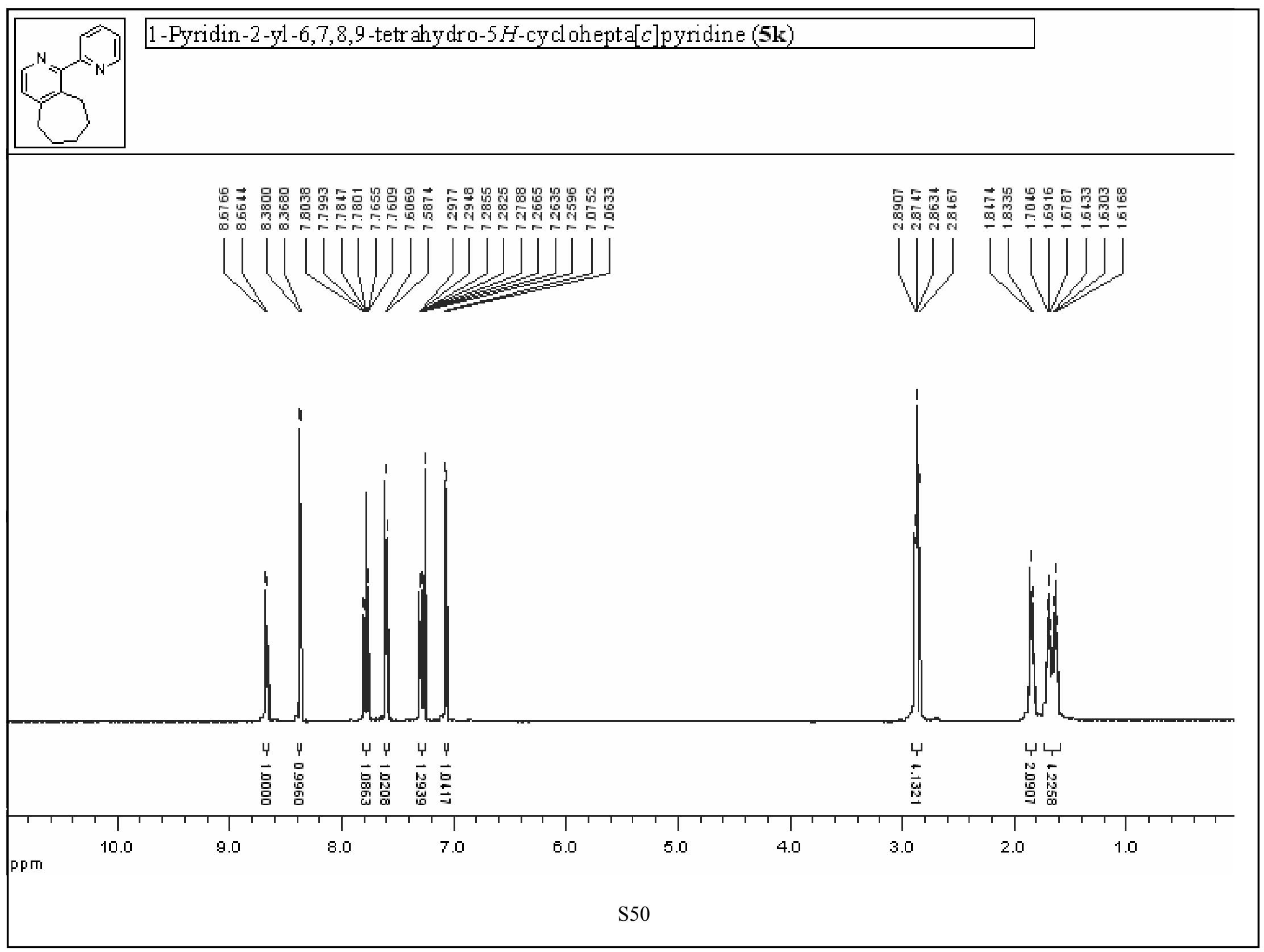




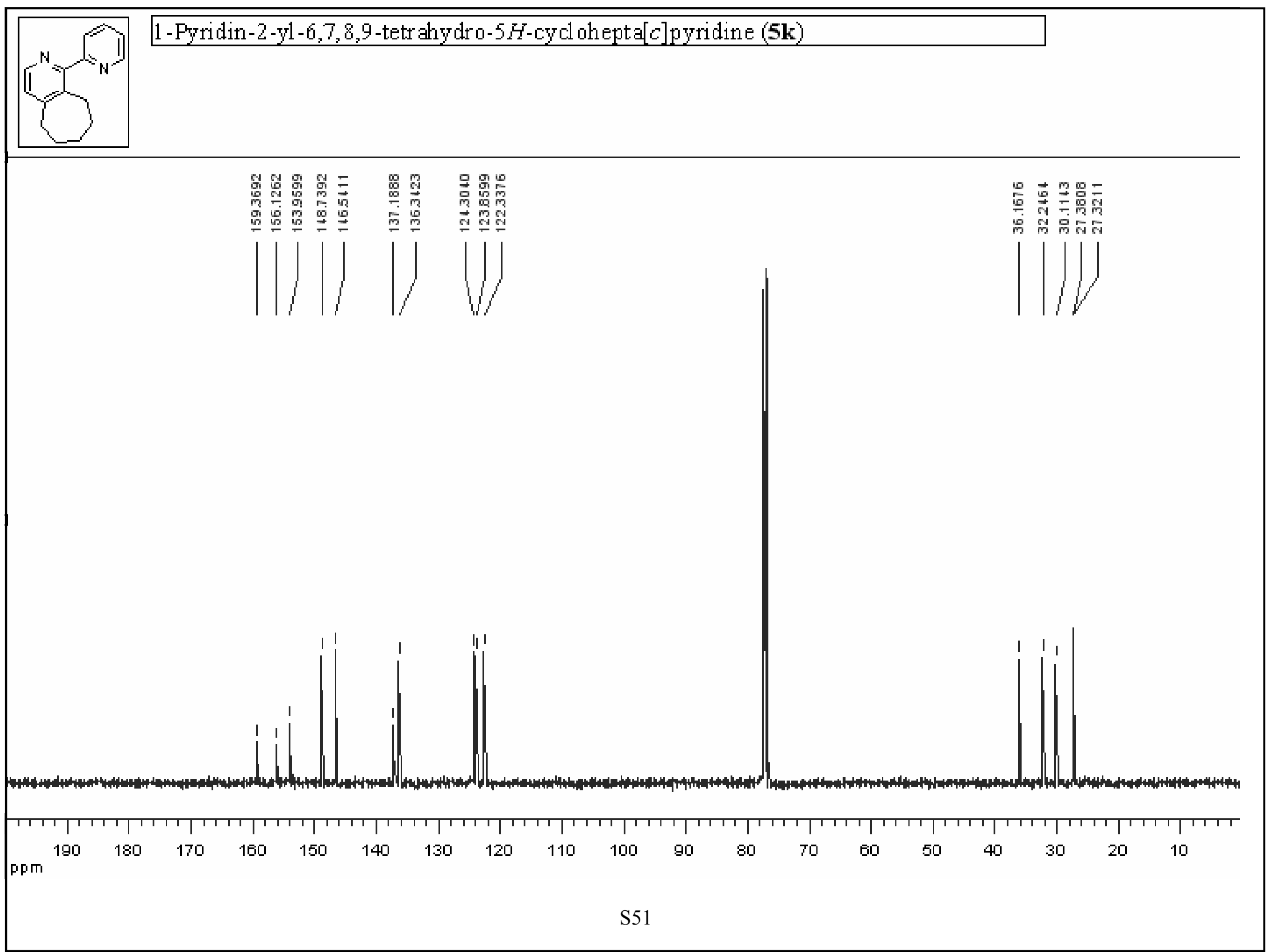




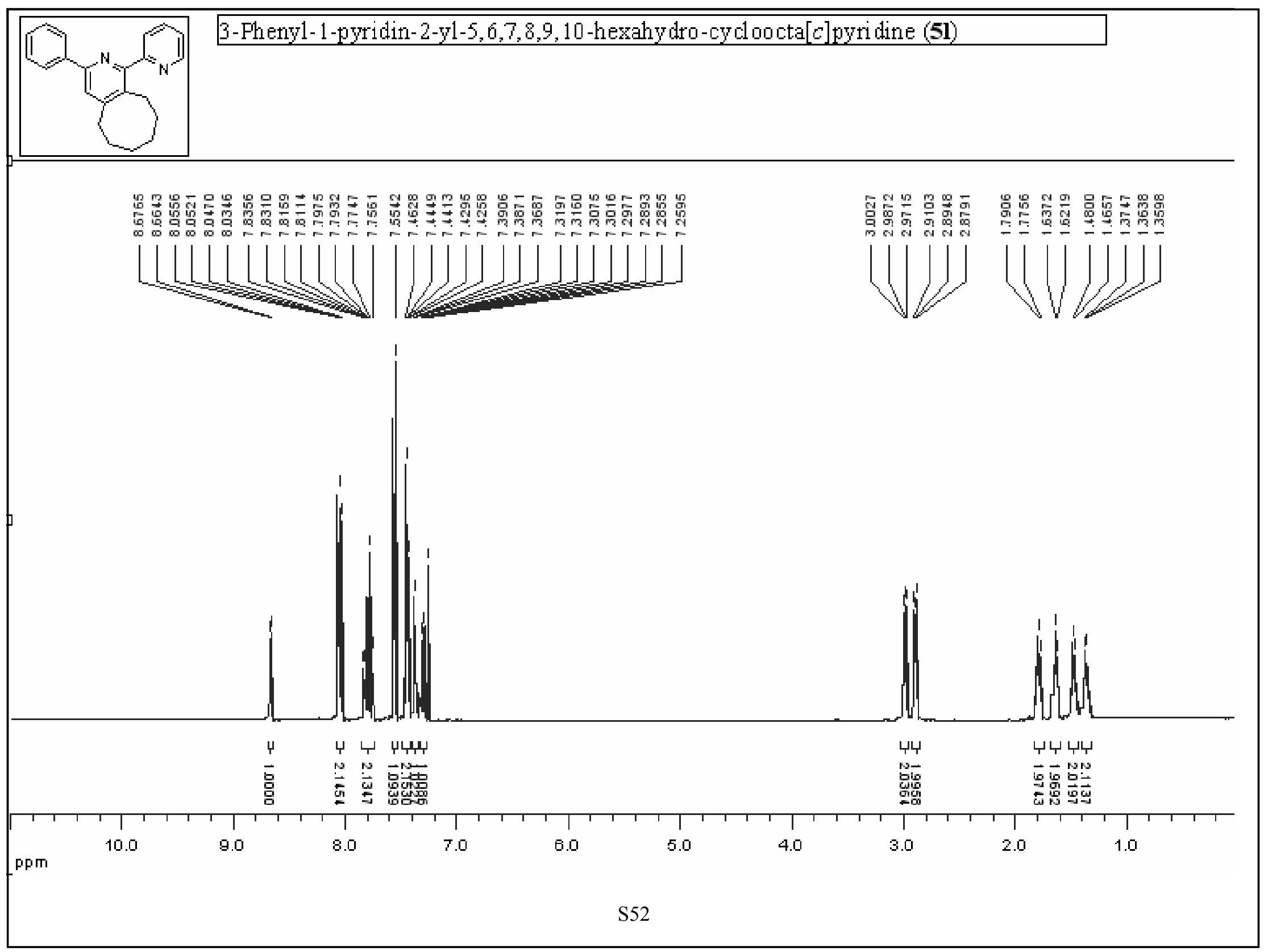




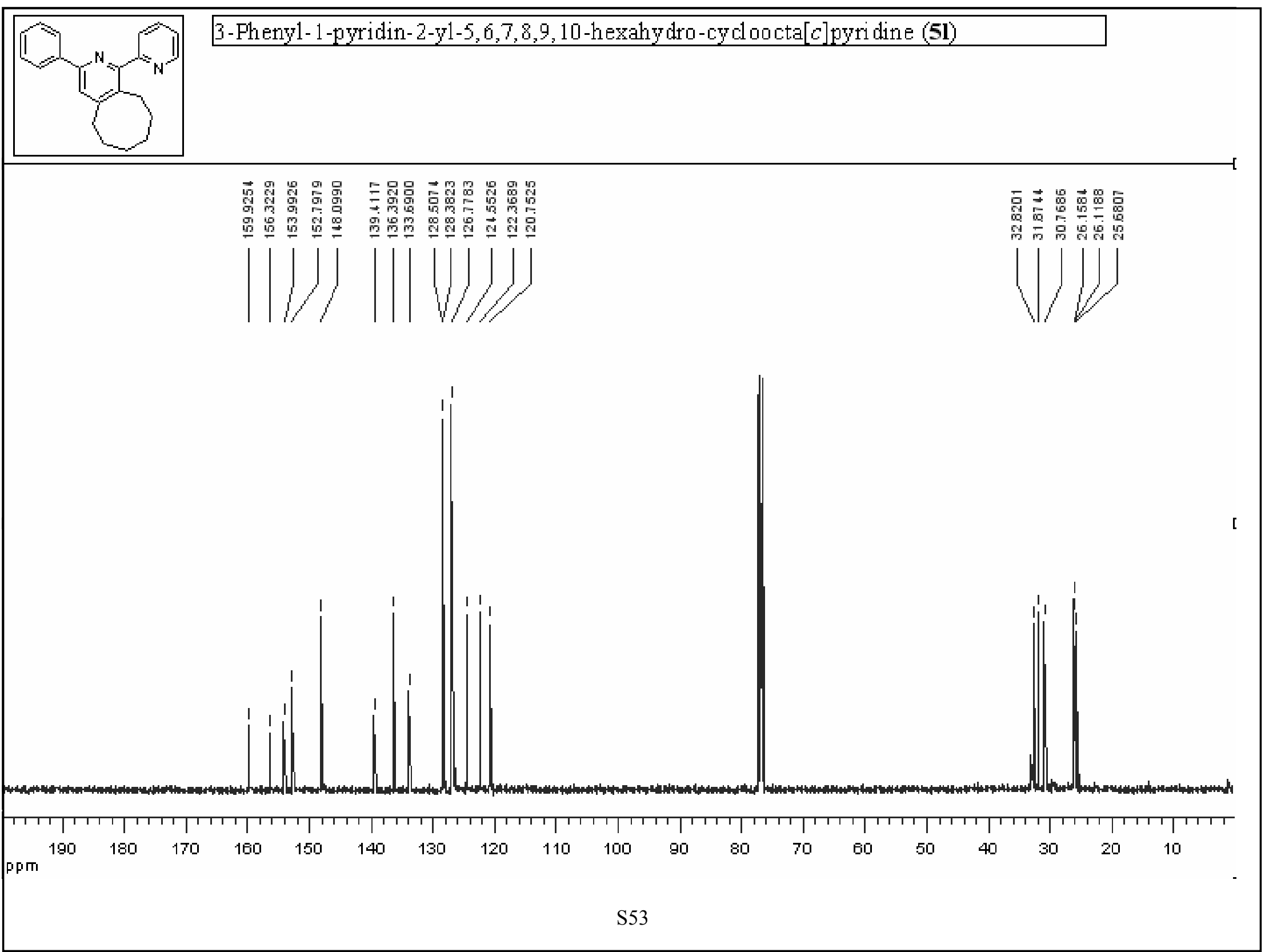




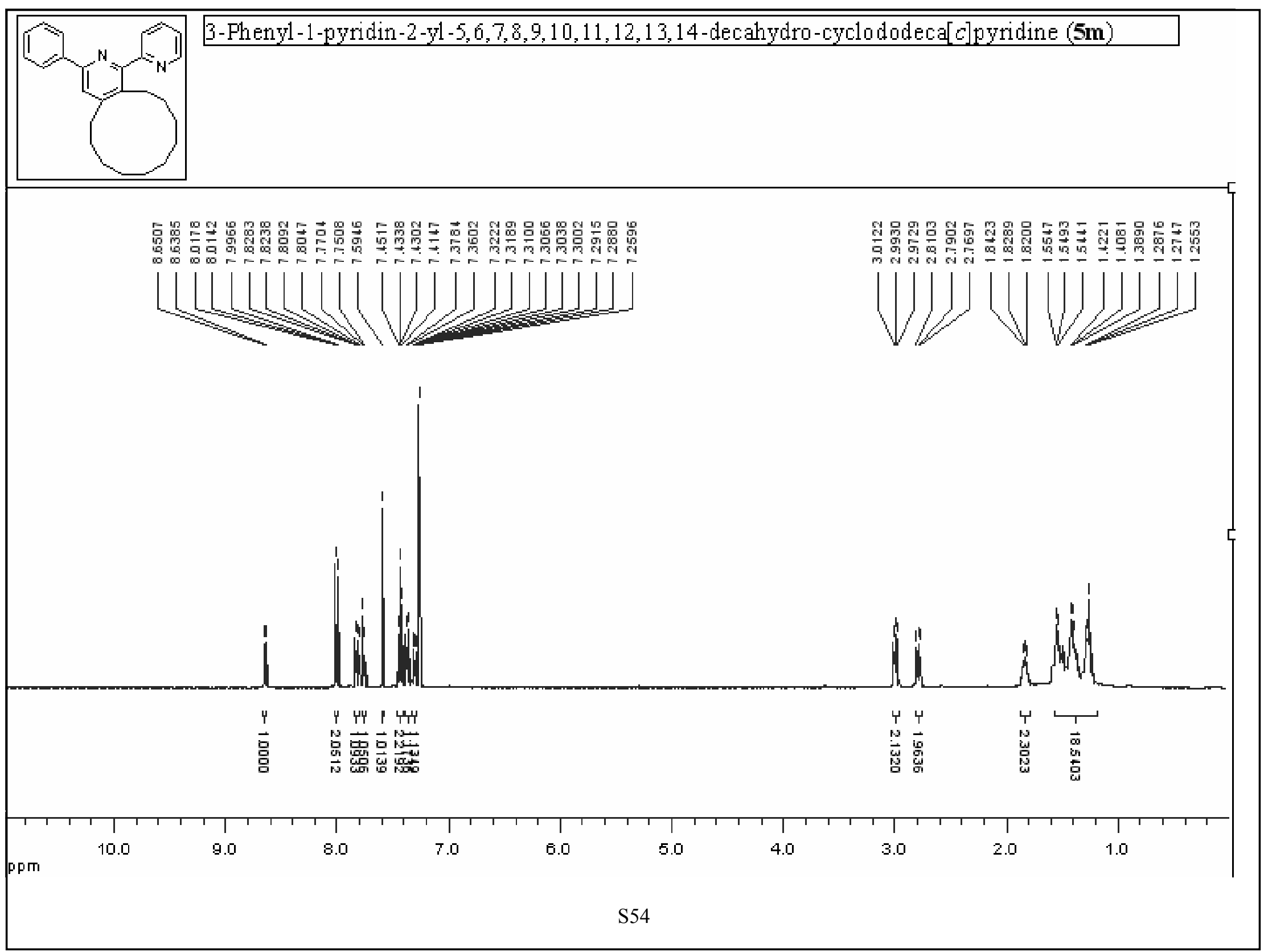




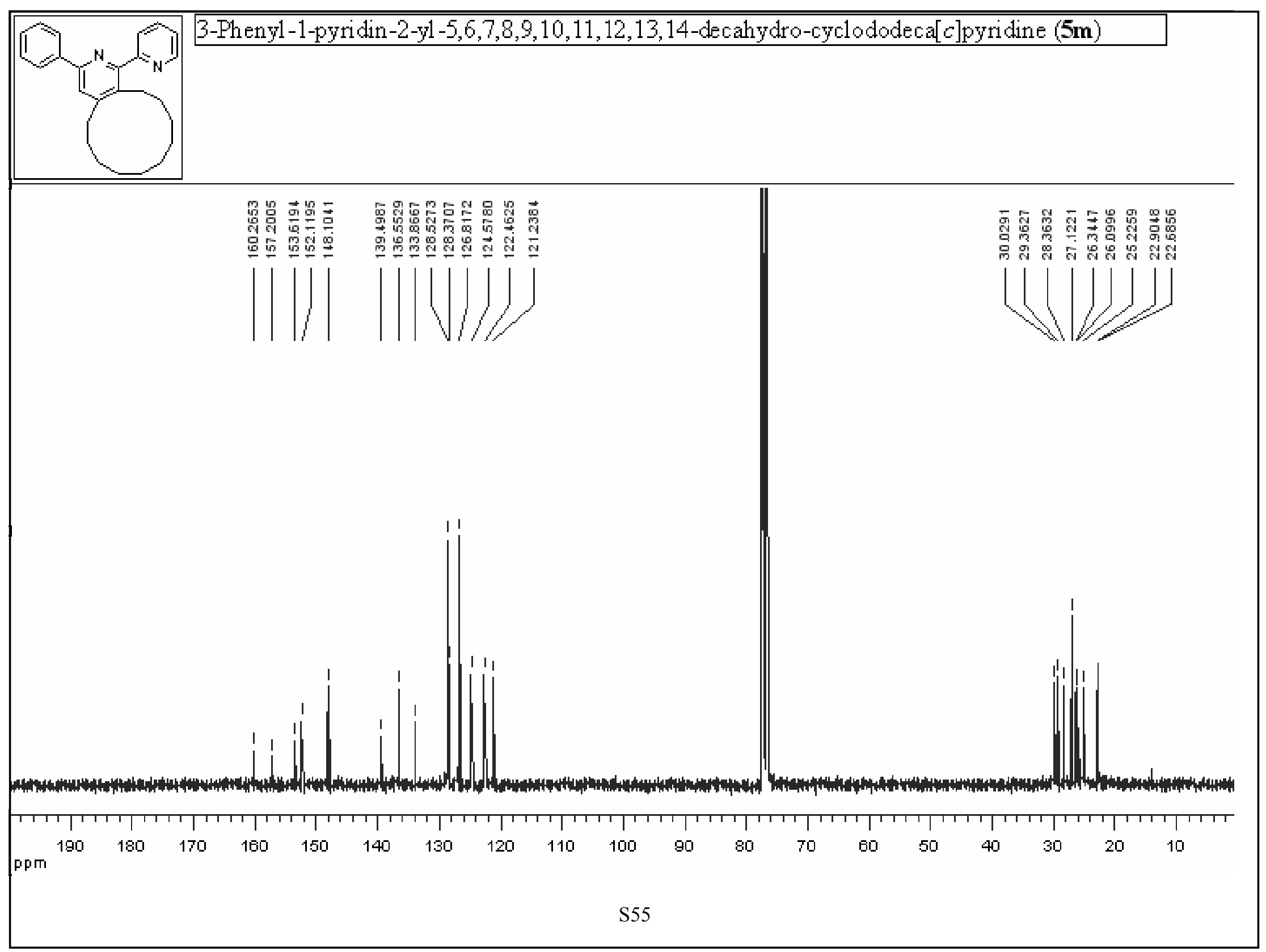




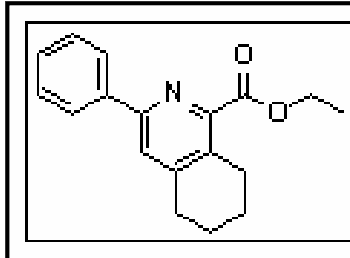

3-Phenyl-5,6,7,8-tetrahydro-isoquinoline-1-carboxylic acid ethyl ester (5n)
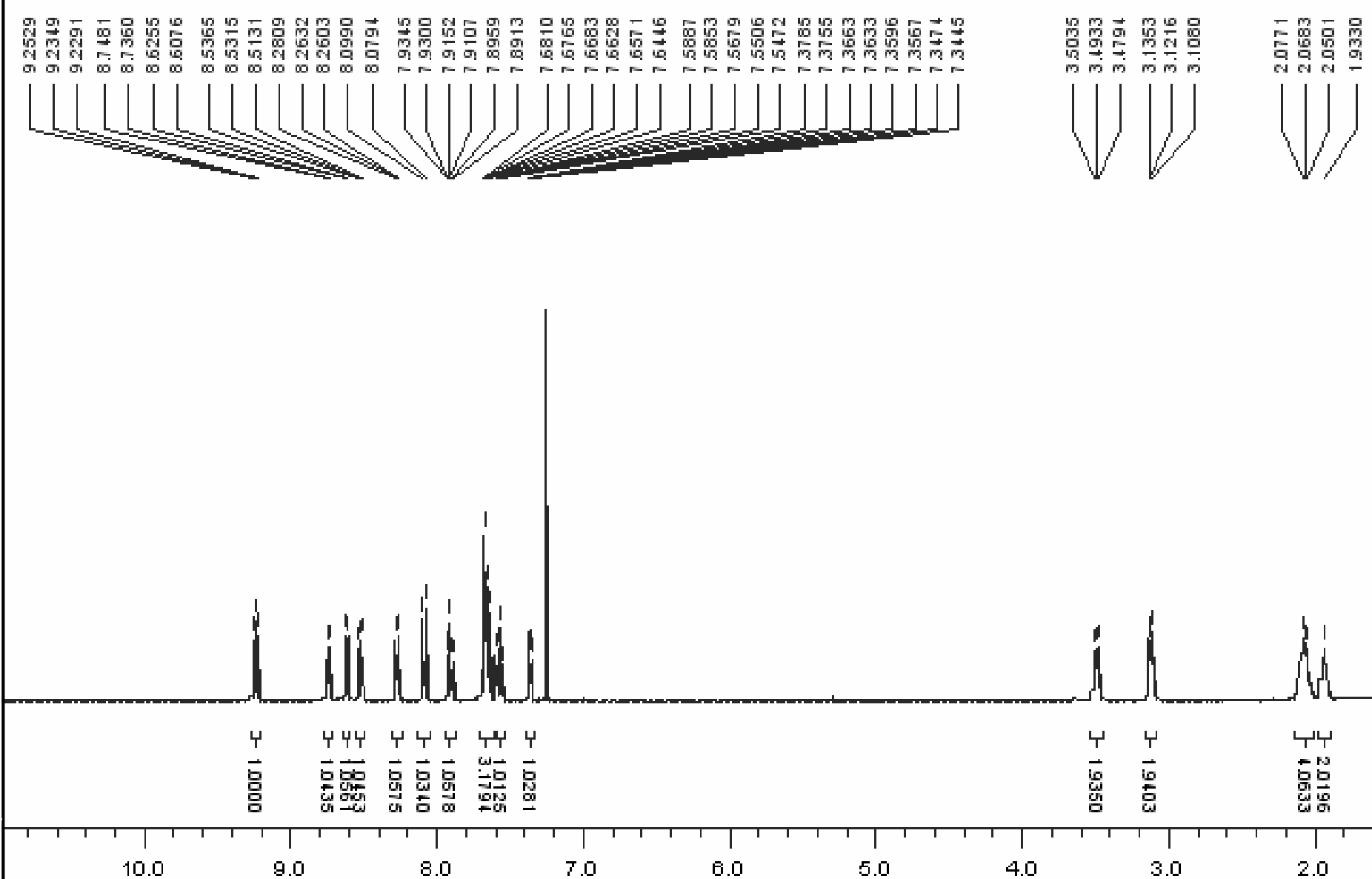

ppm

9.0

8.0

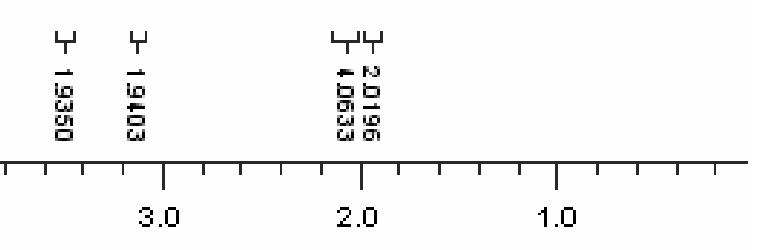




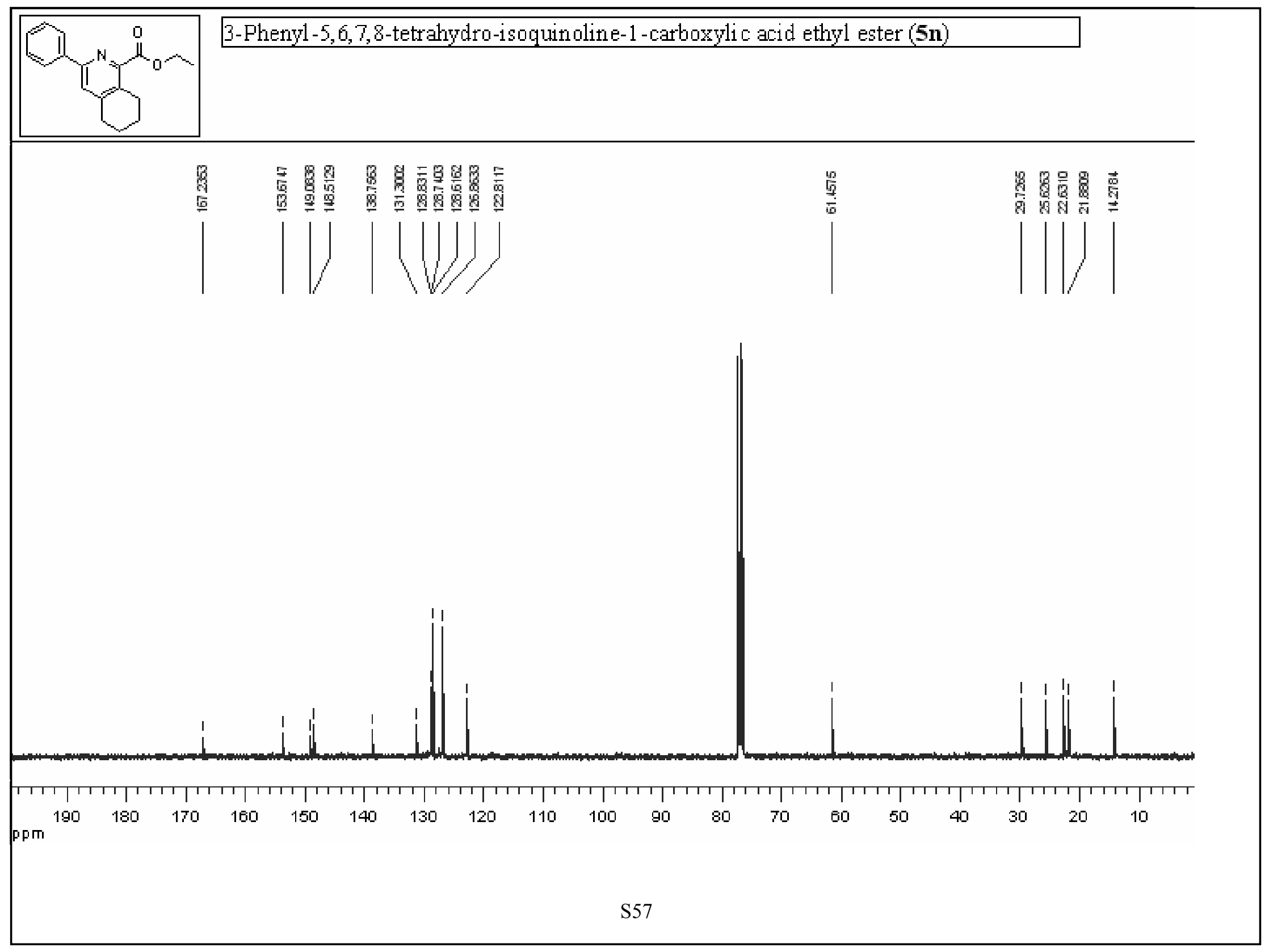




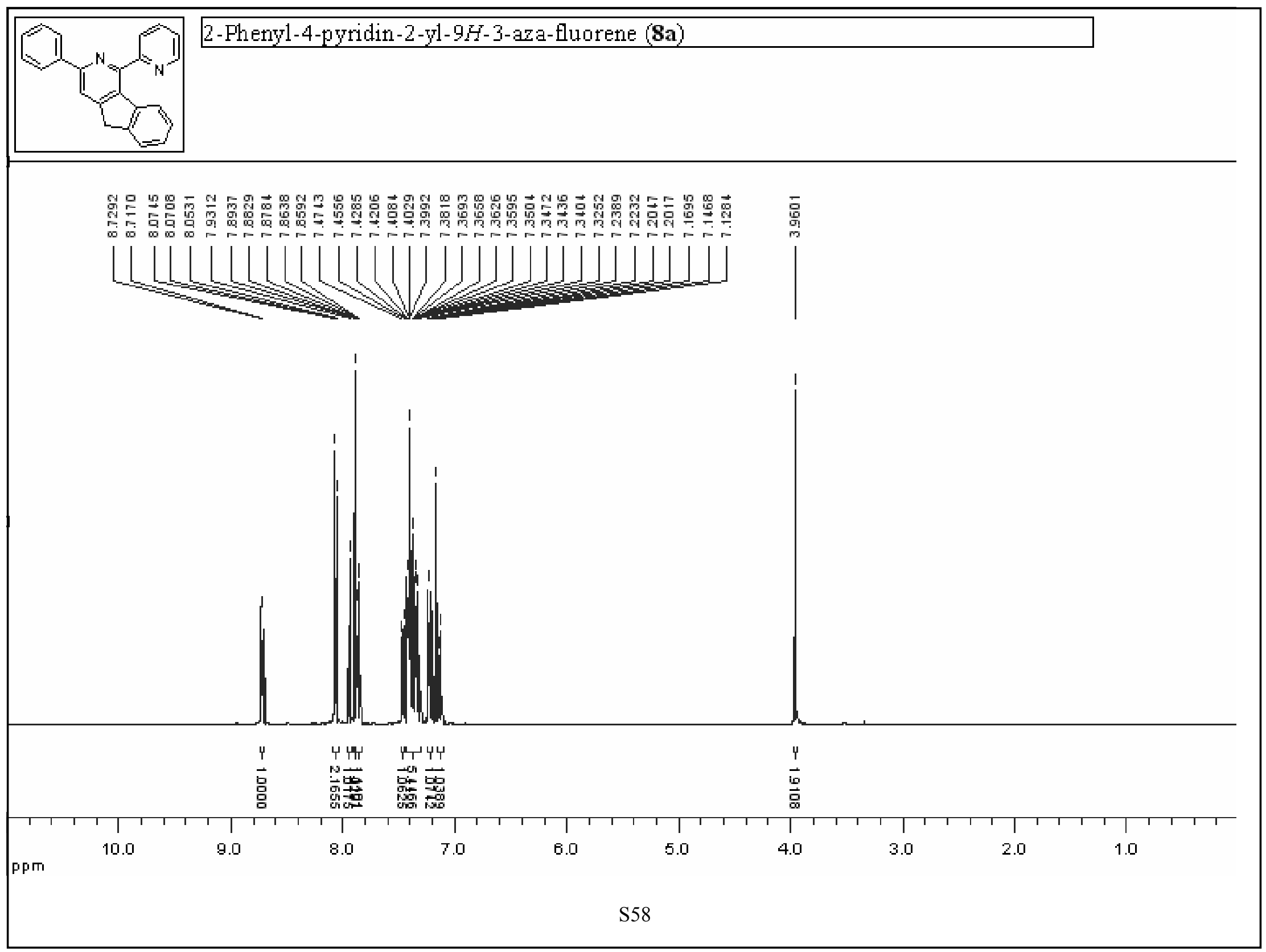




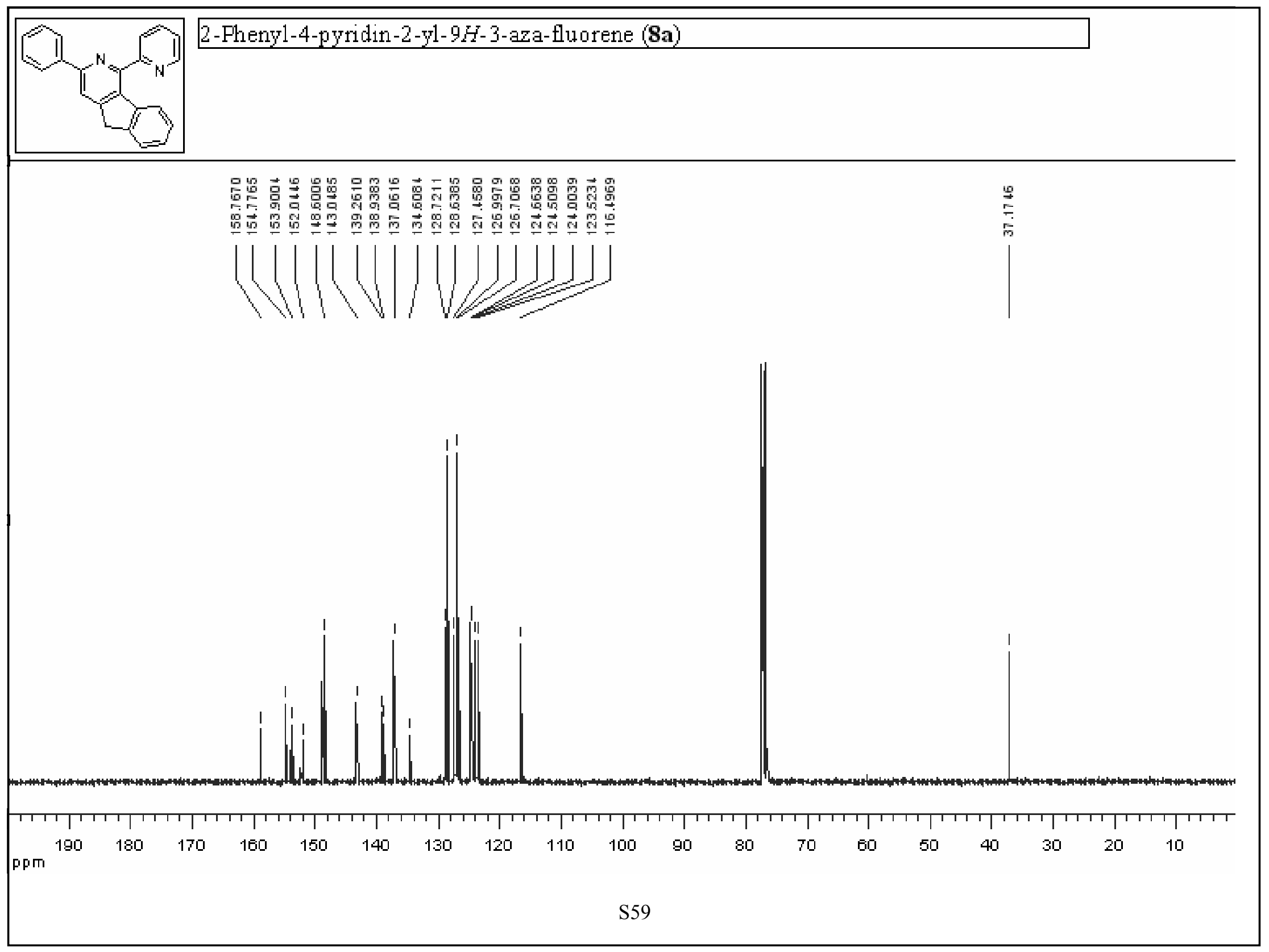




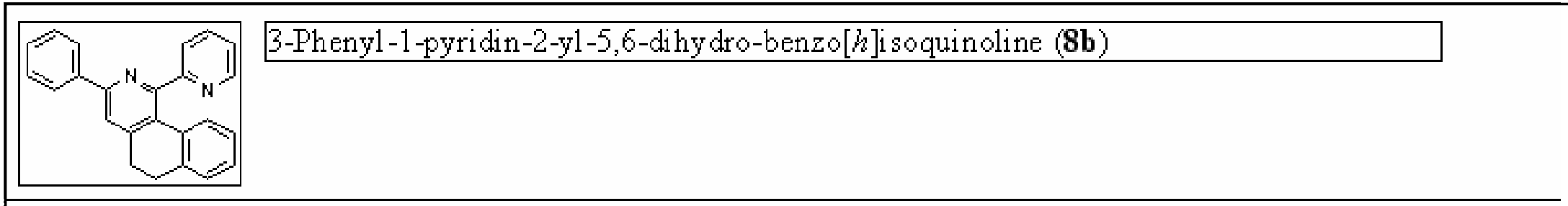

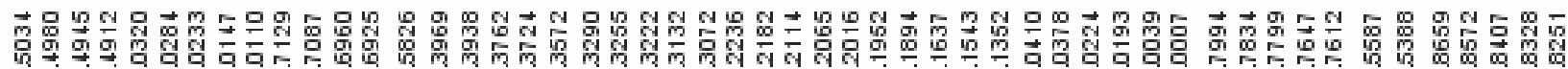
w

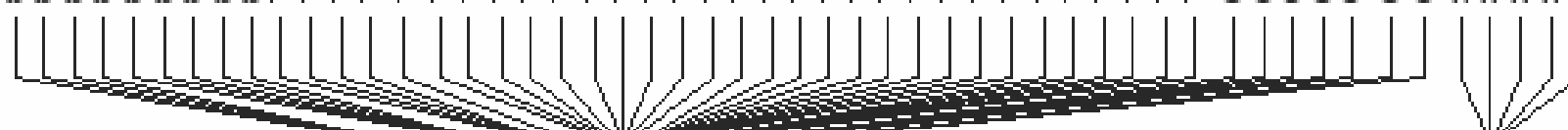

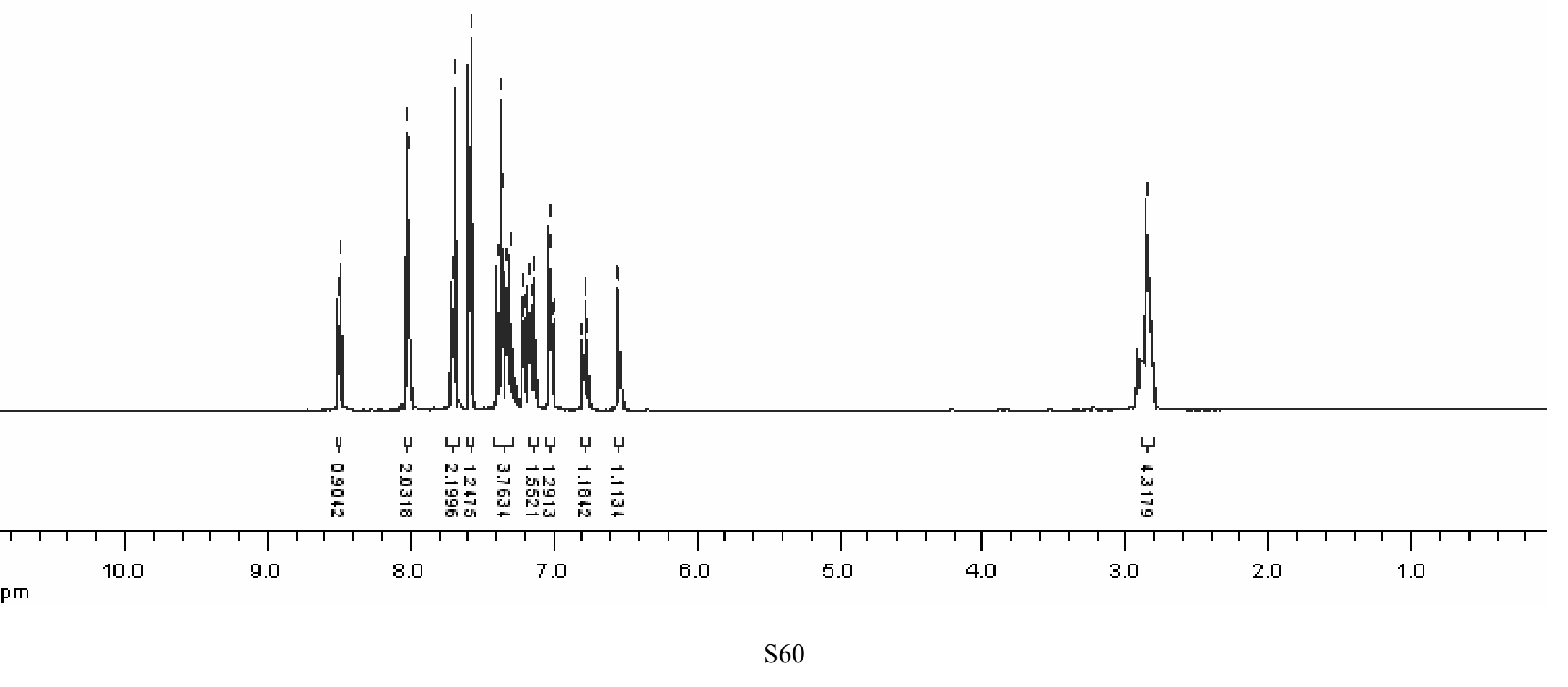




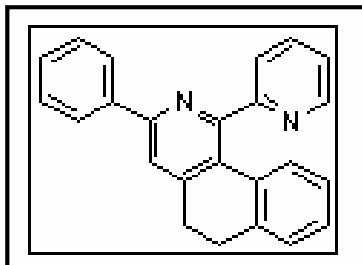

3-Phenyl-1-pyridin-2-yl-5,6-dihydro-benzo[h]isoquinoline (8b)

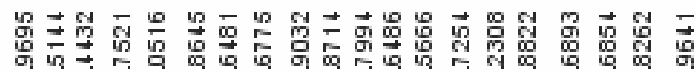
盟
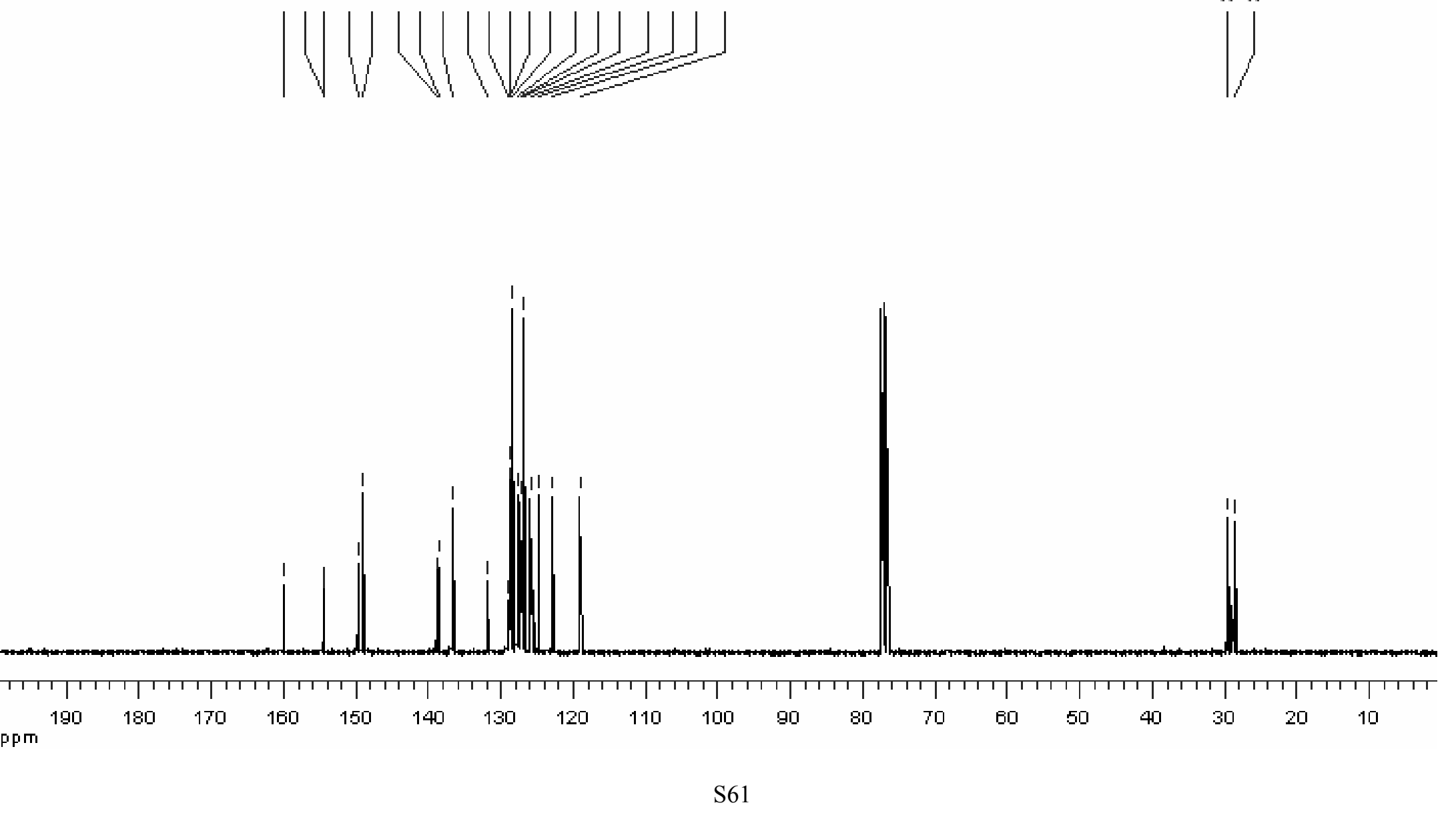


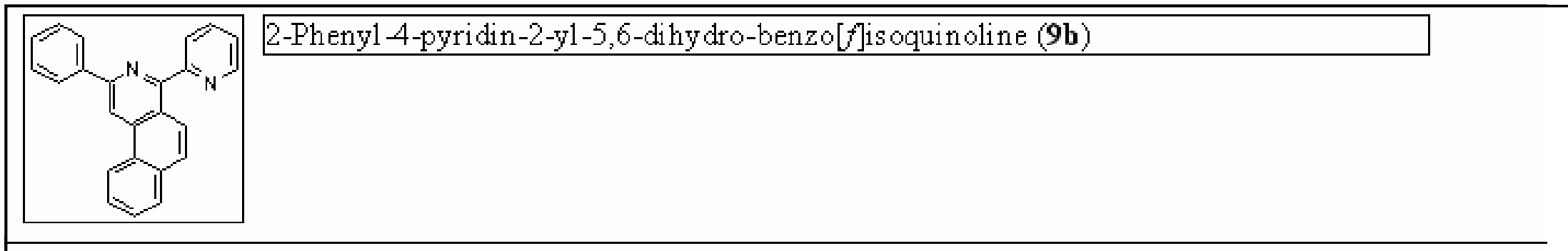

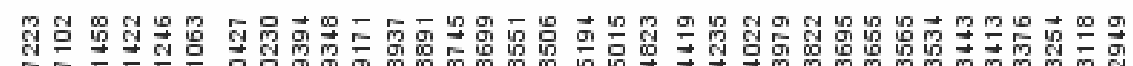

范

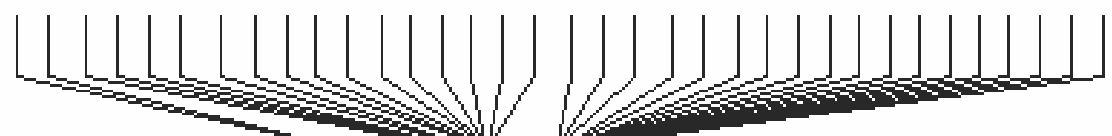
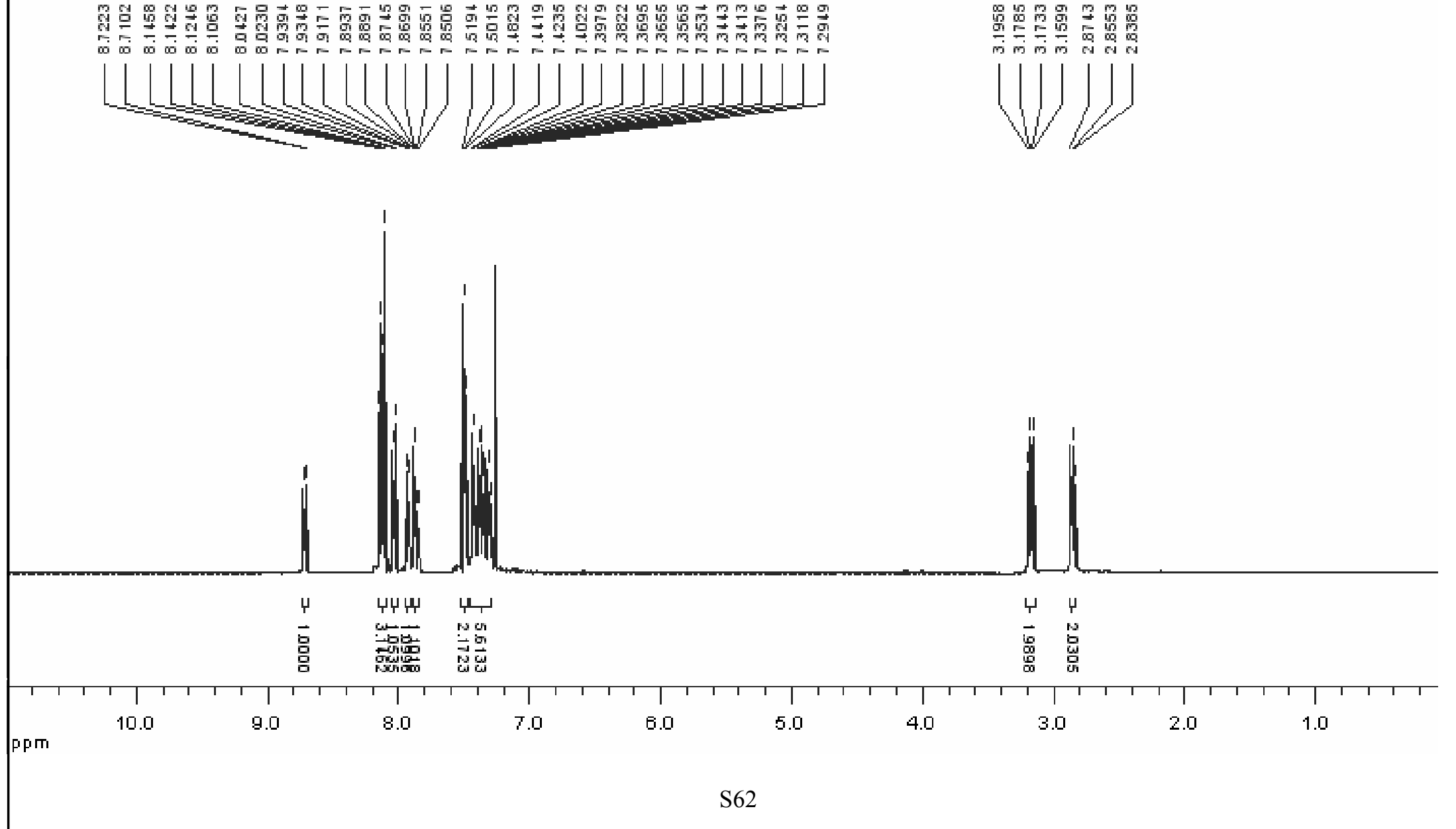


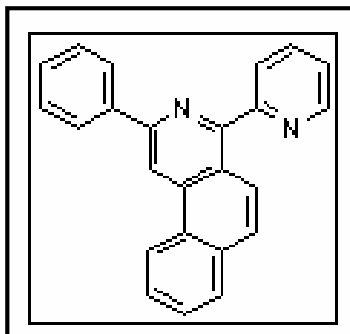

\section{2-Phenyl-4-pyridin-2-yl-5,6-dihydro-benzo[f] isoquinoline (9b)}

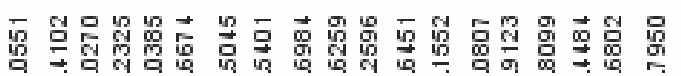

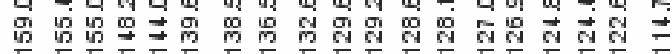
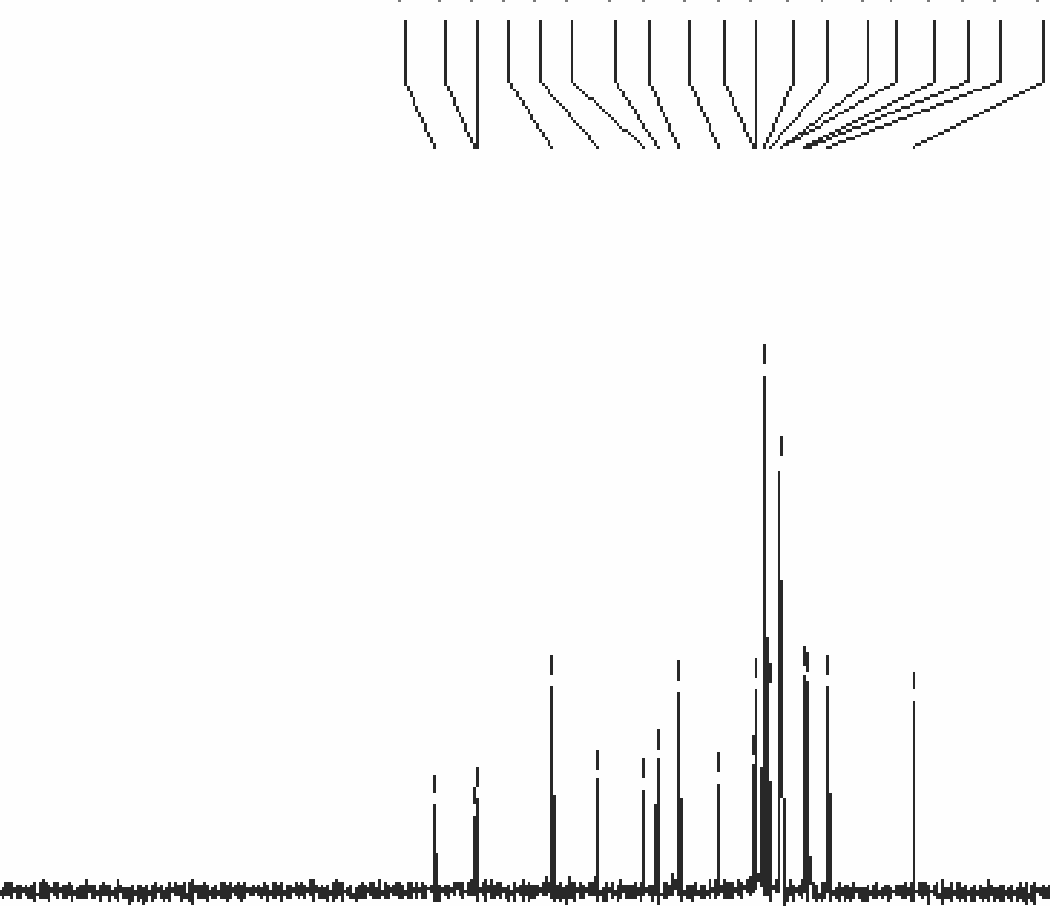

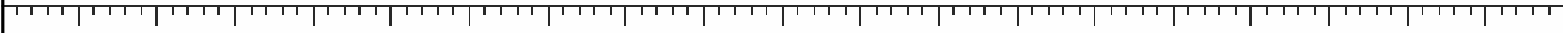
$\mathrm{ppm}$

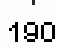

180

170

160150

140

130

120

110100

90

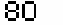

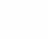

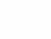

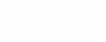

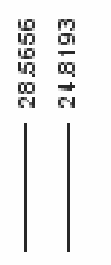




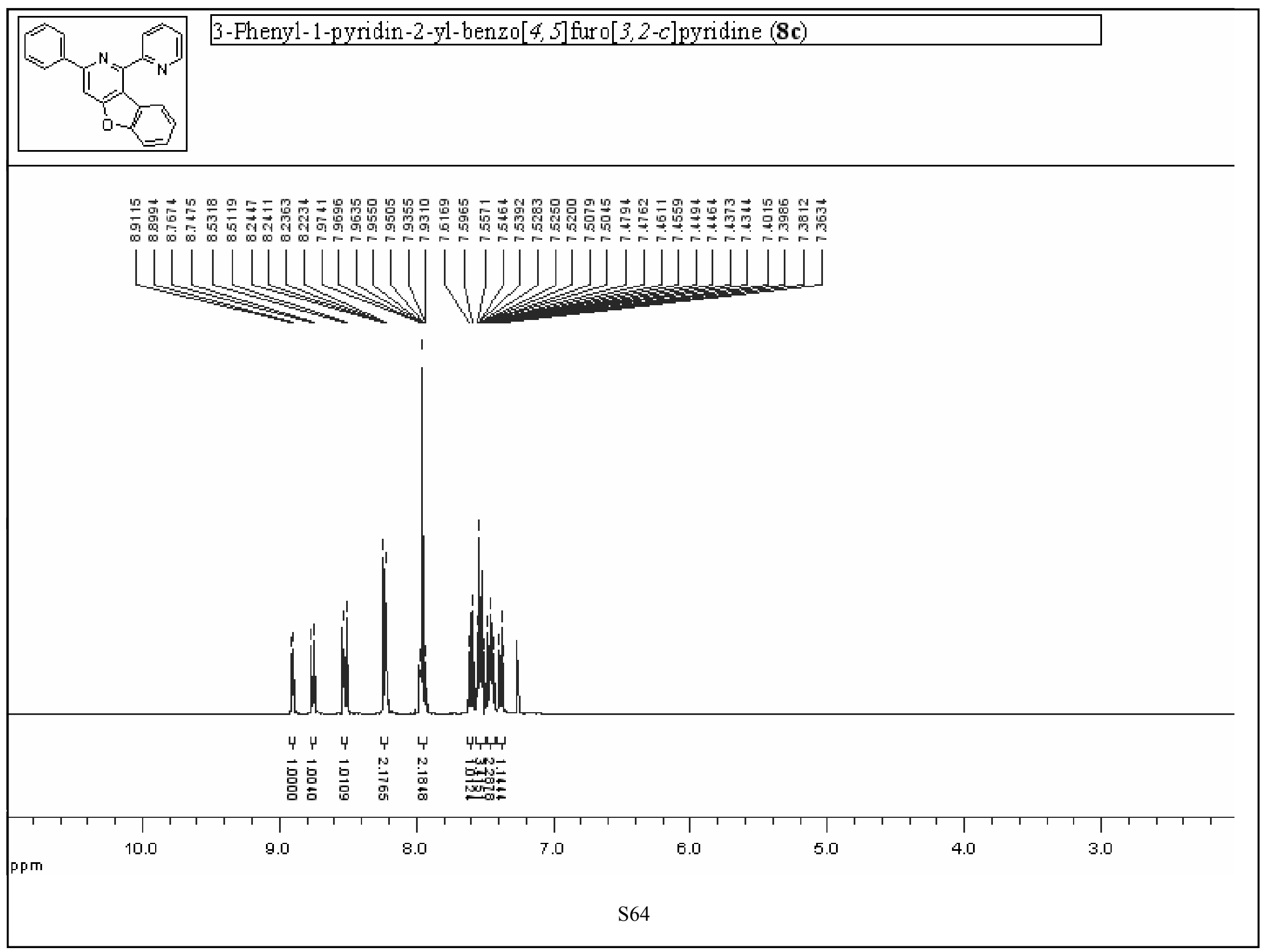




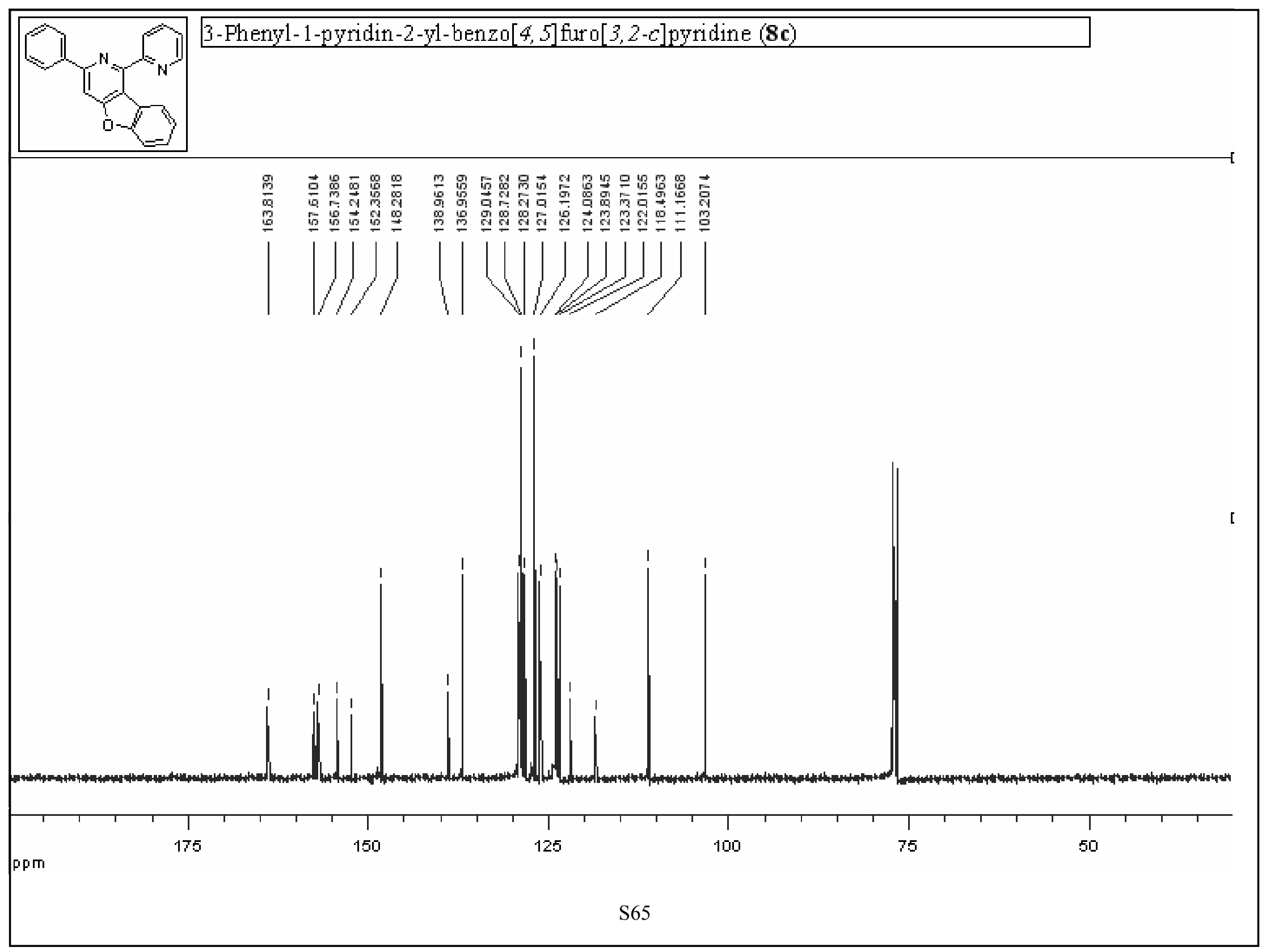




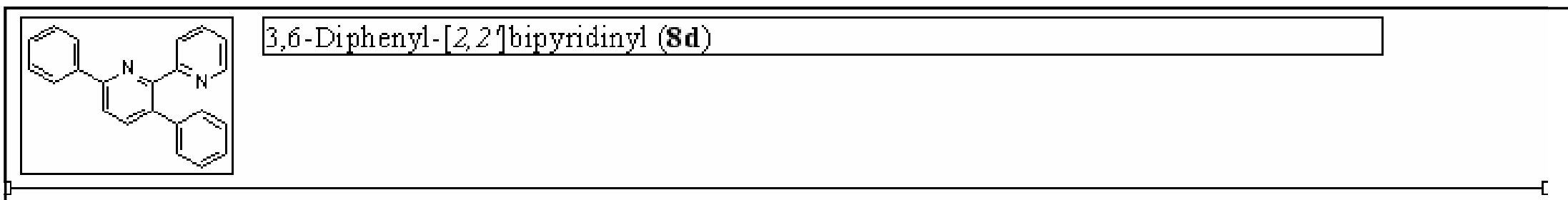

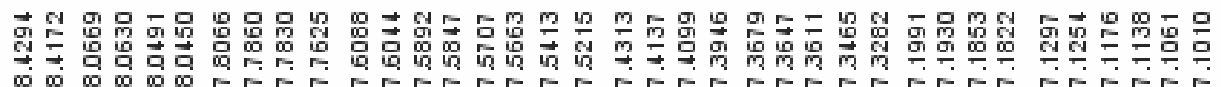

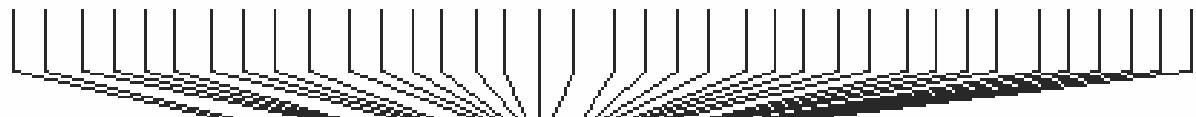
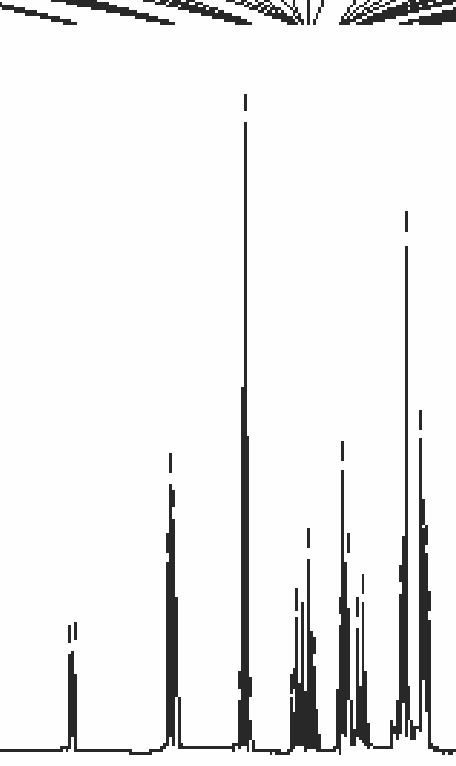

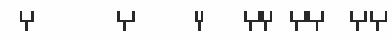

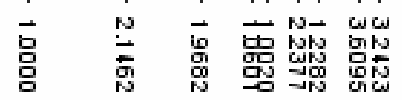

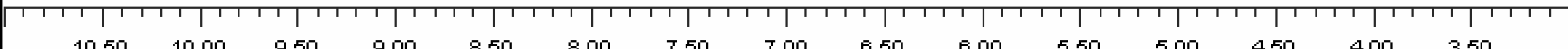

0.50




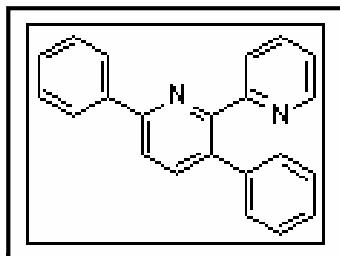

3,6-Diphenyl-[2,2]bipyridinyl (8d)

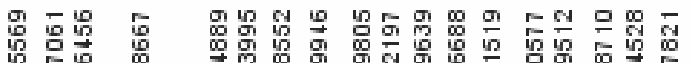

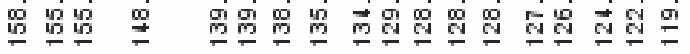

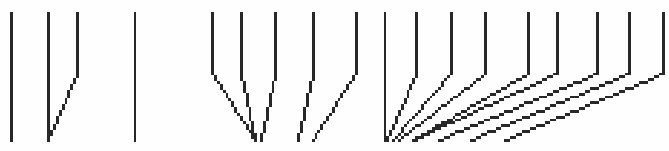

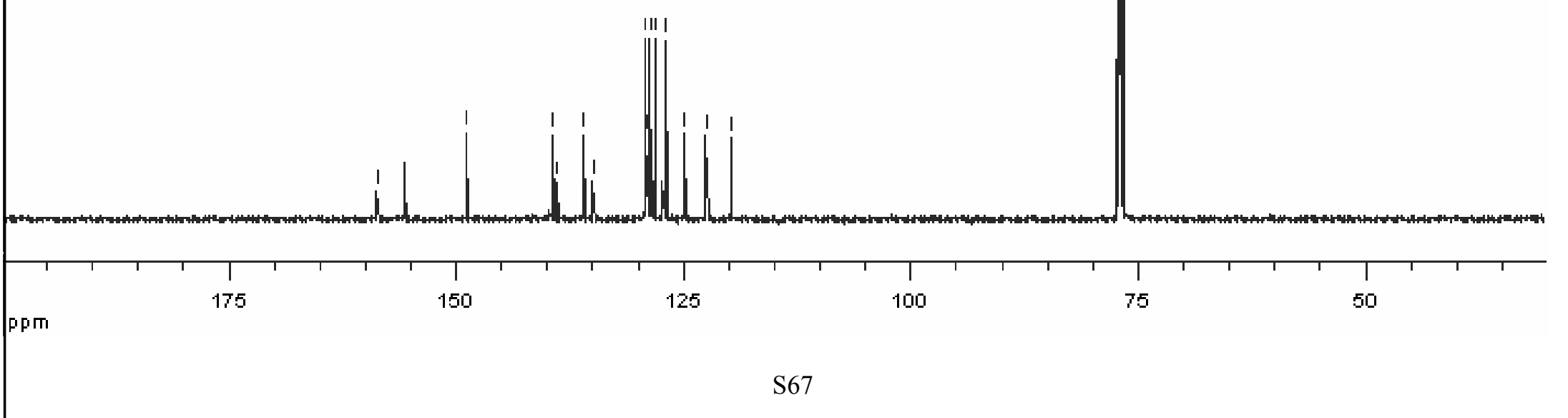




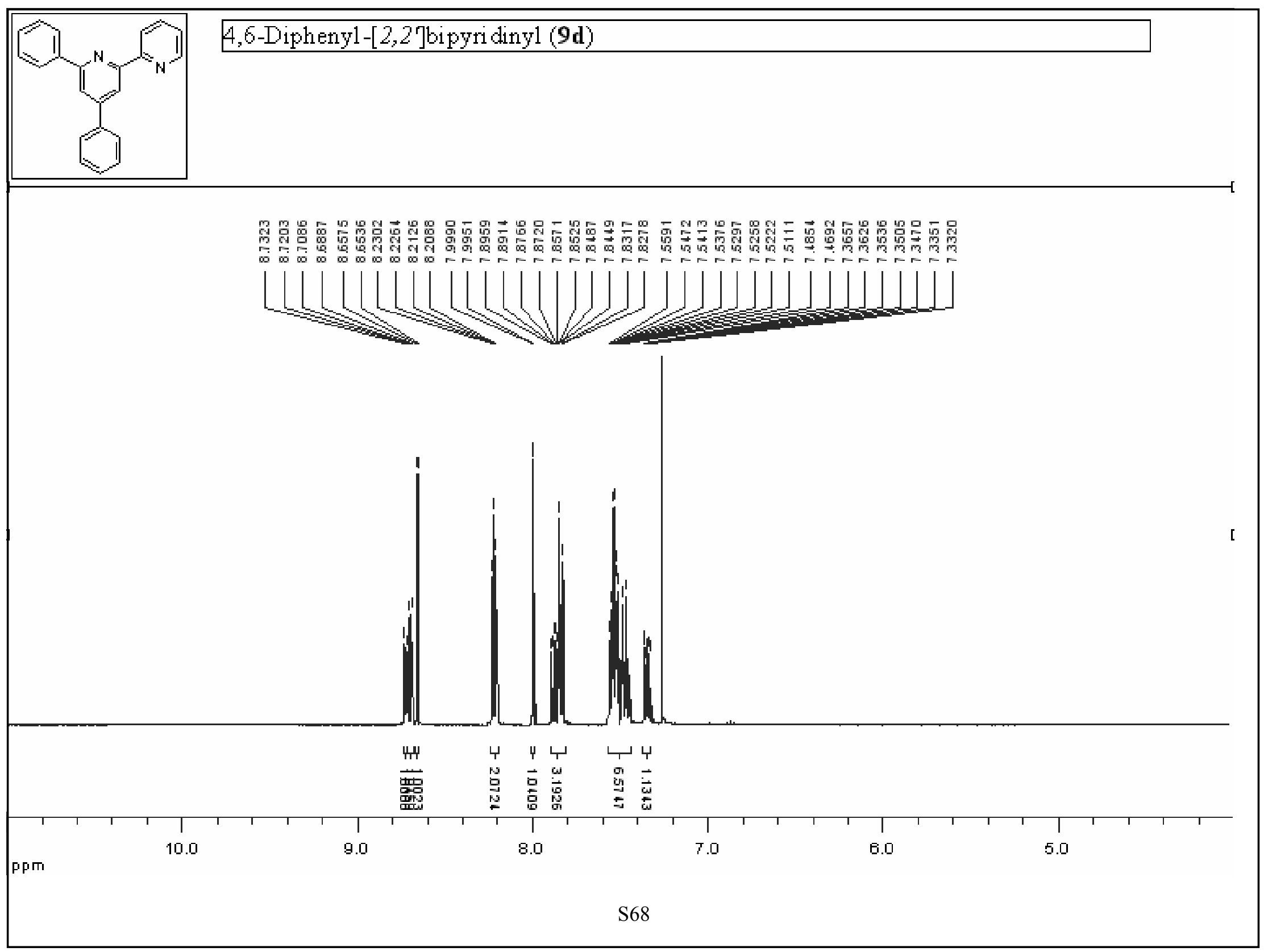




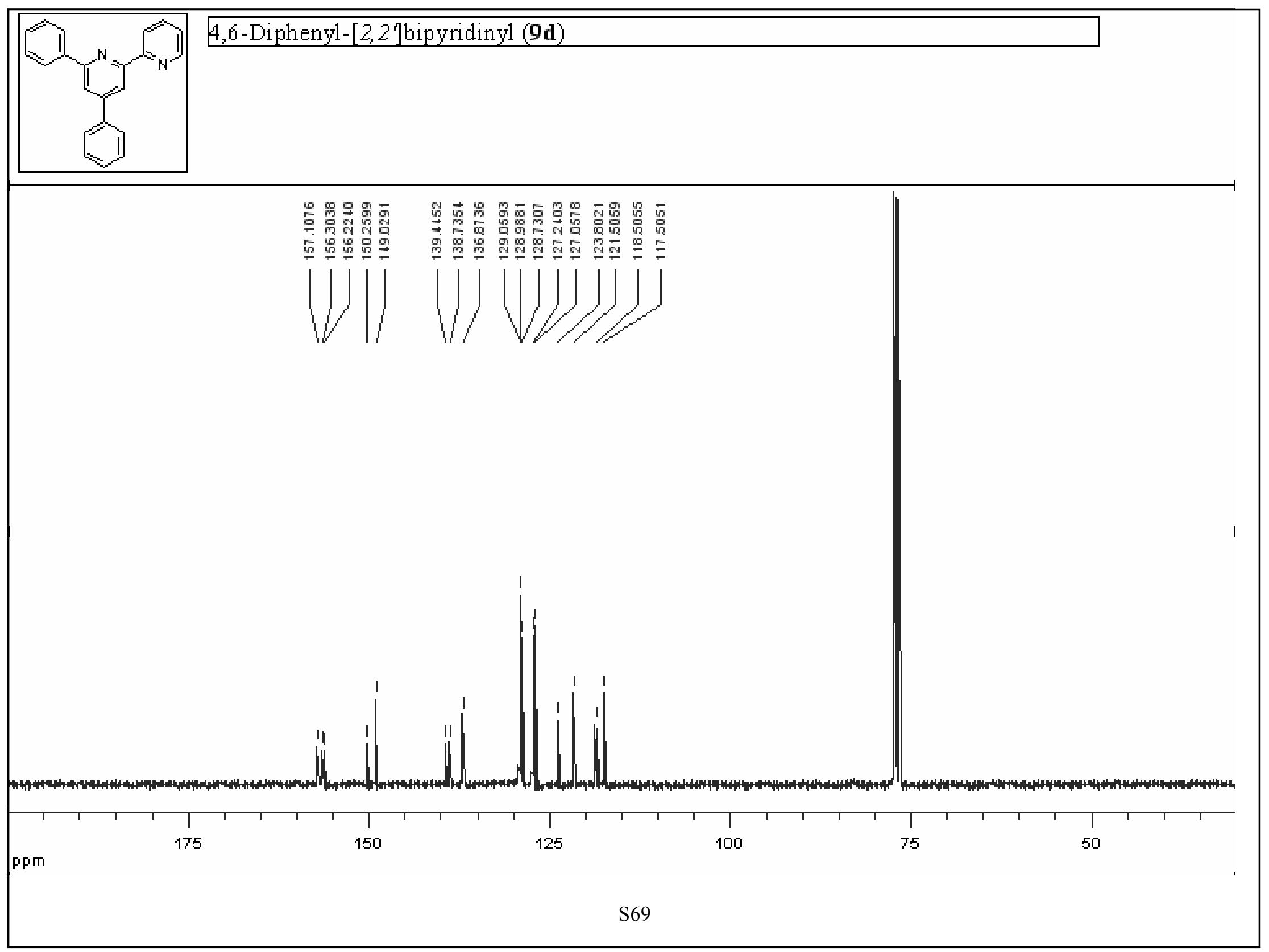




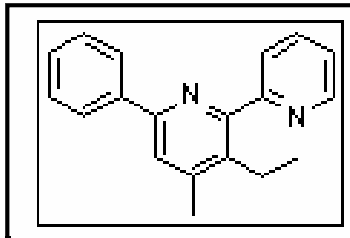

3-Ethyl 4-methyl-6-phenyl-[2,2']bipyri dinyl (8e)

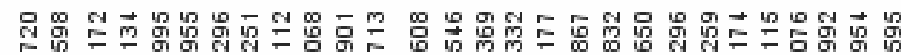

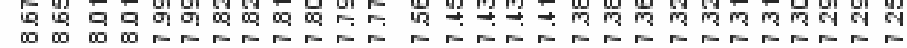

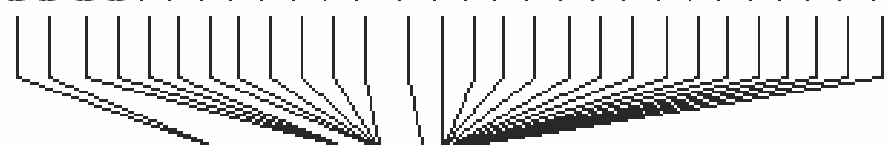
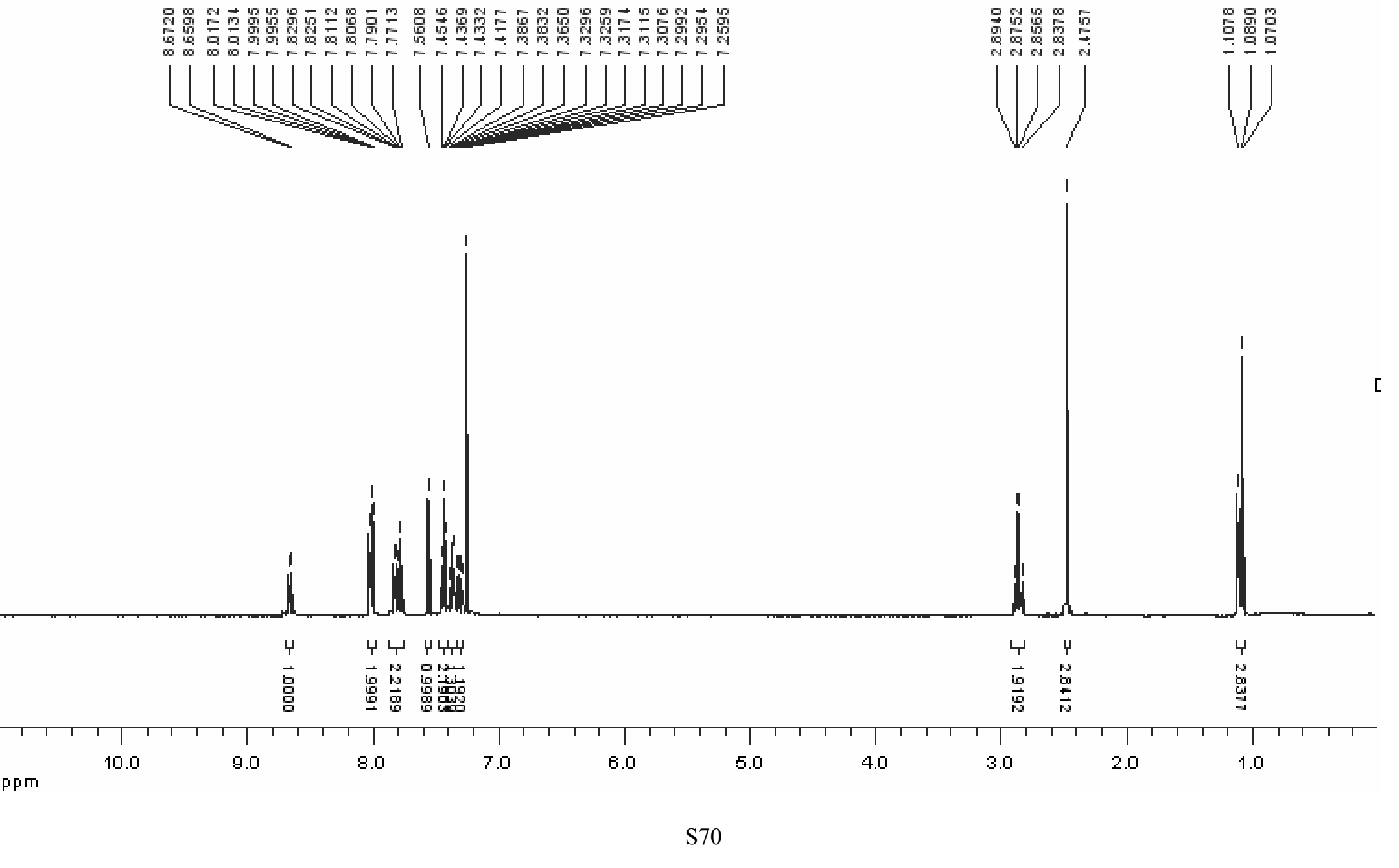


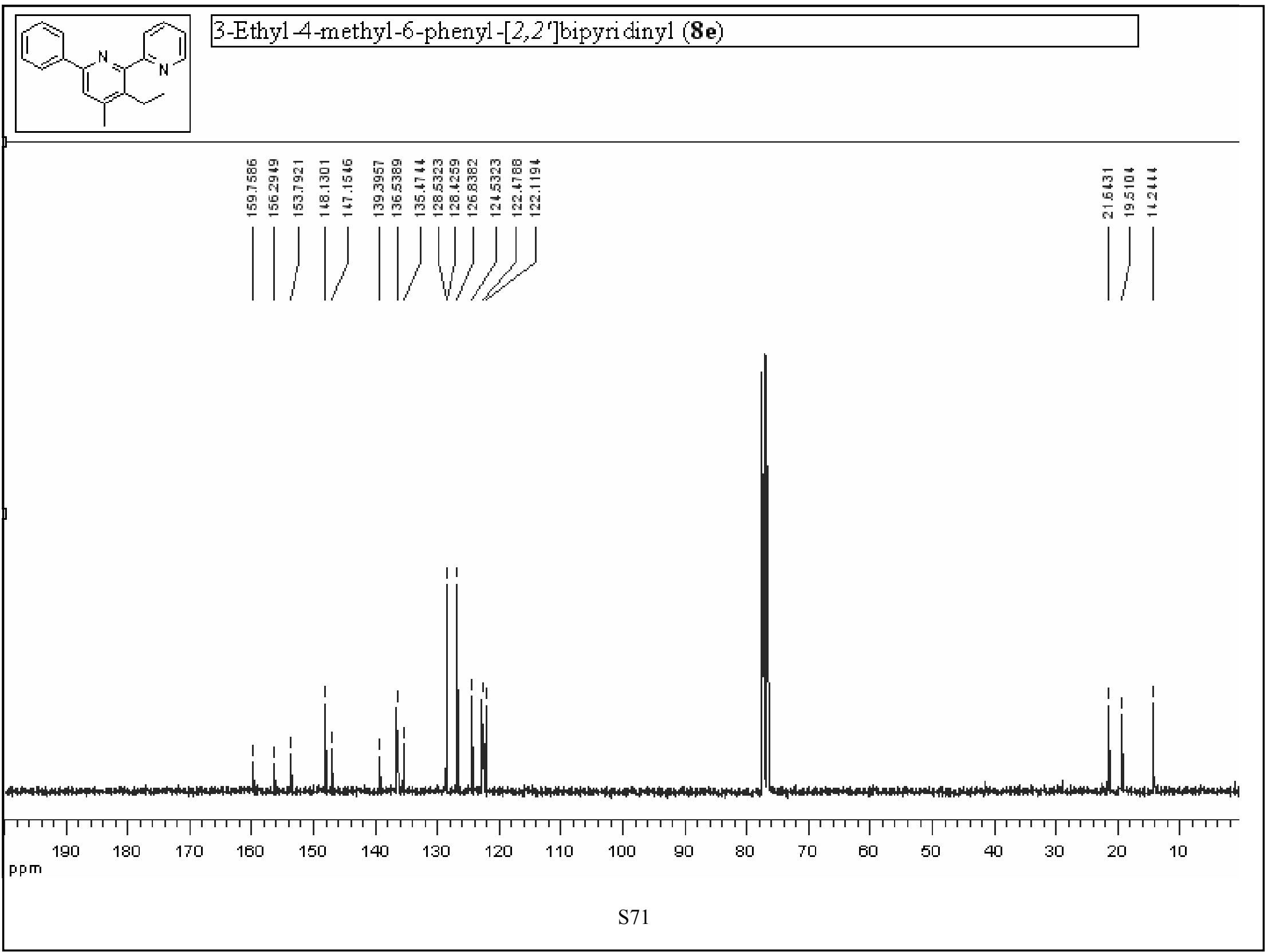




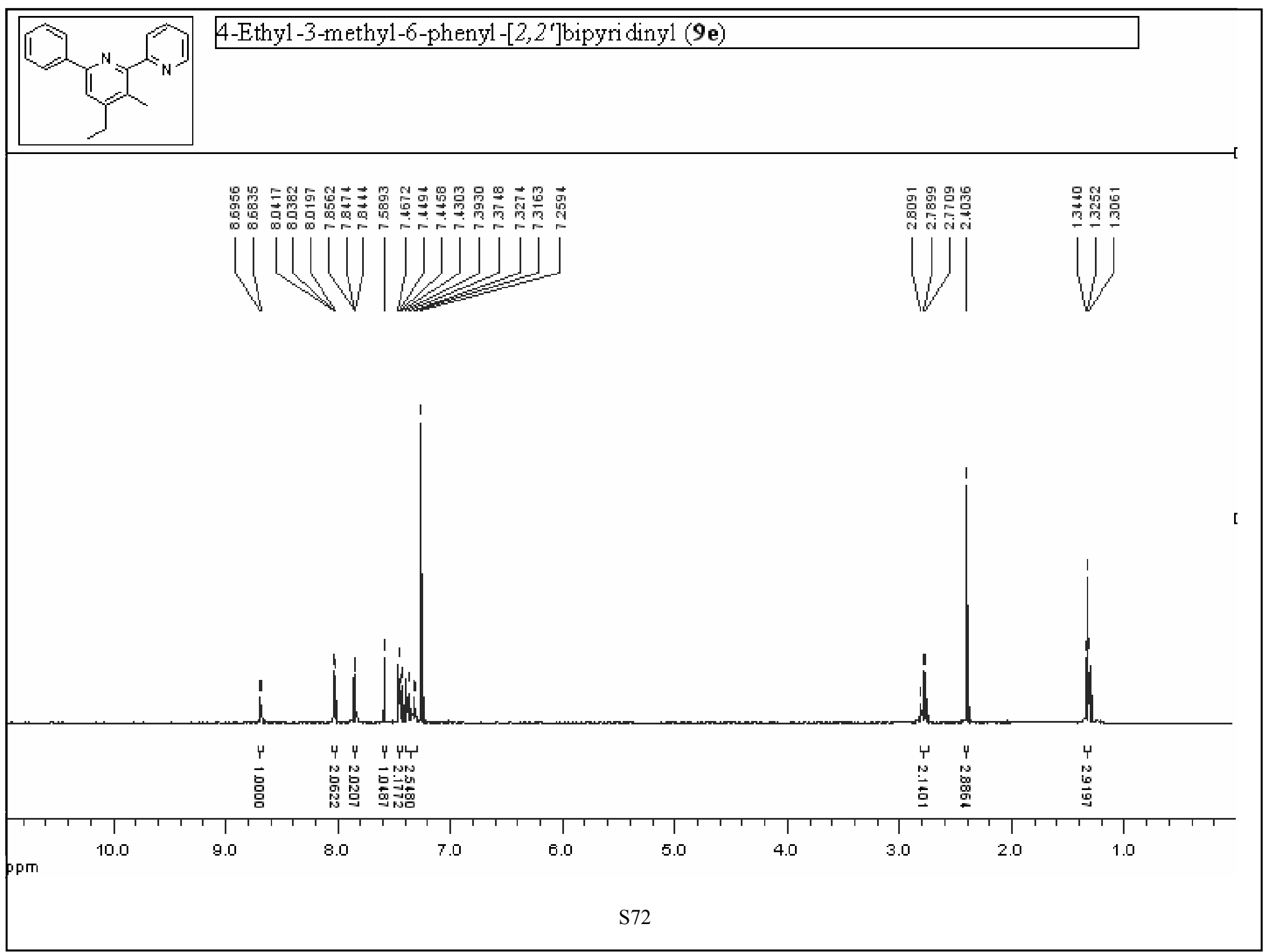




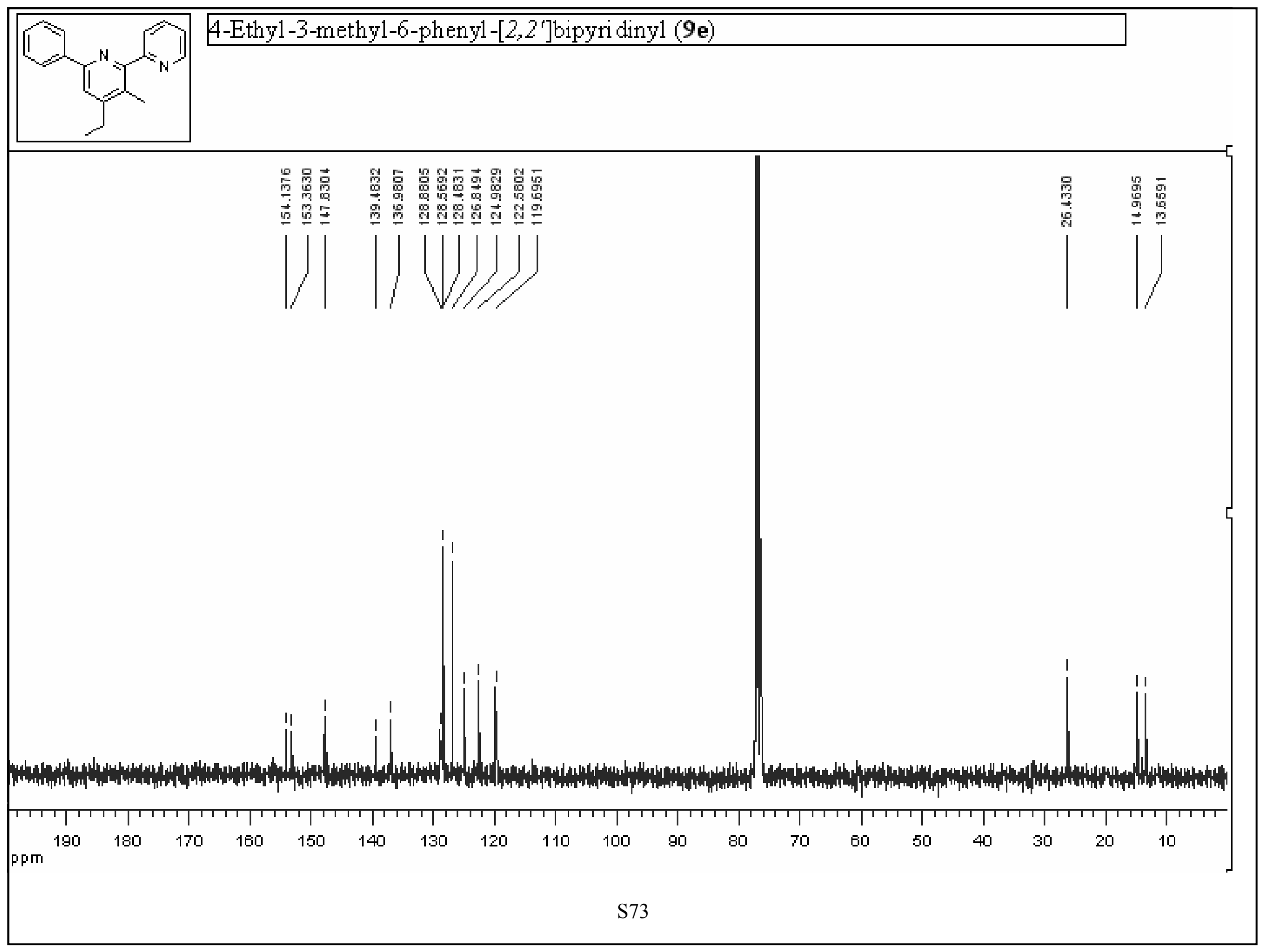




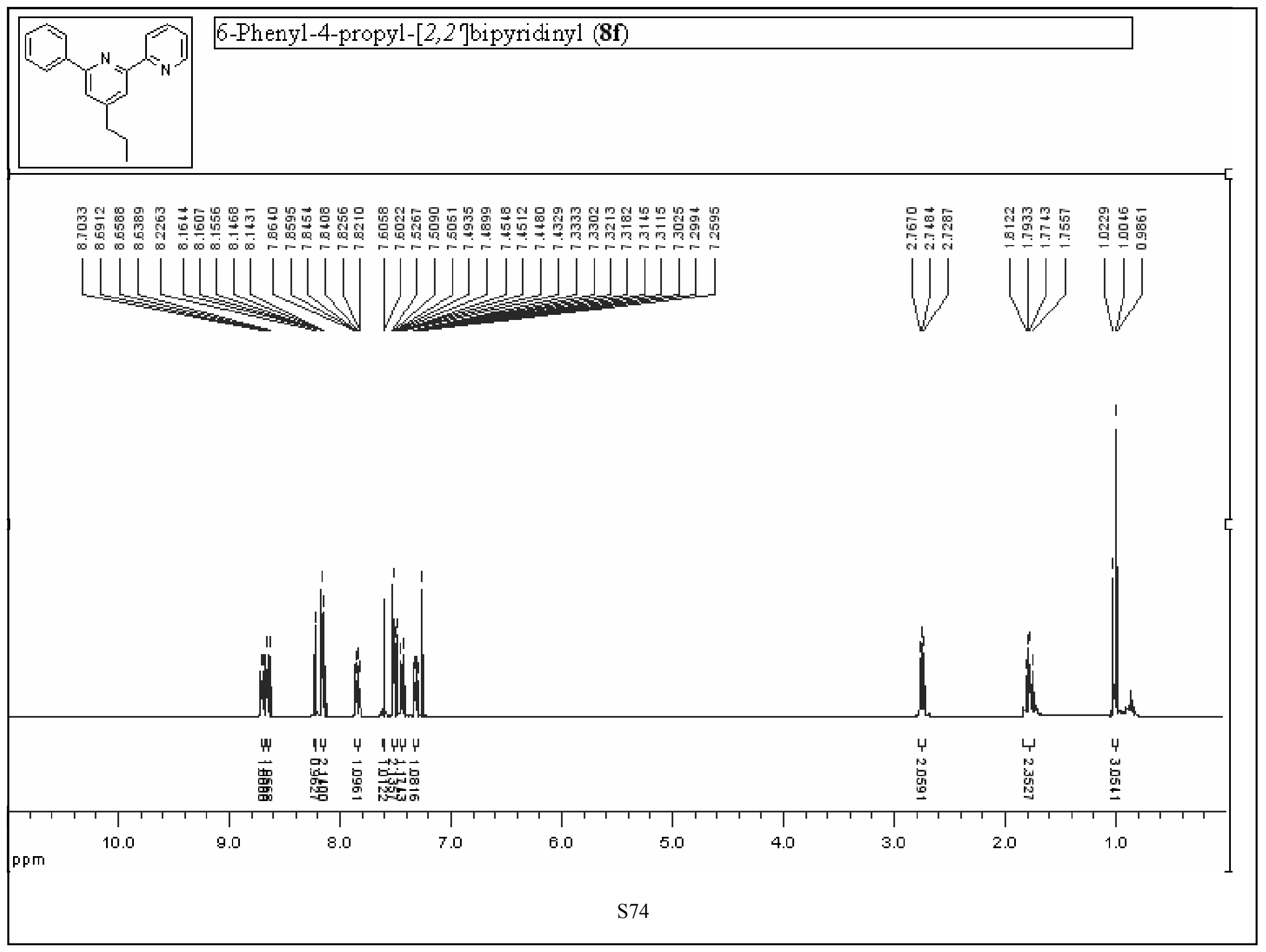




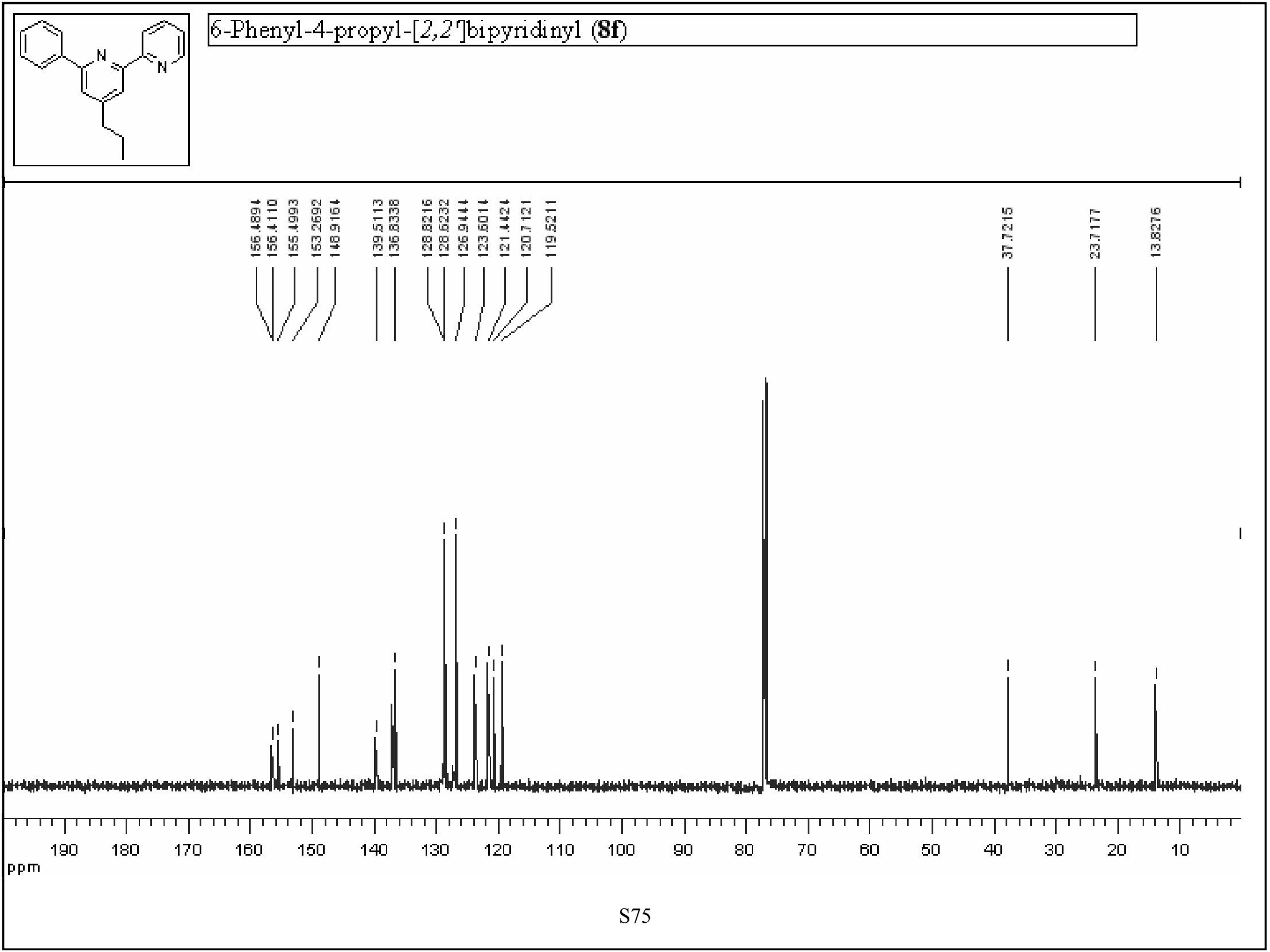




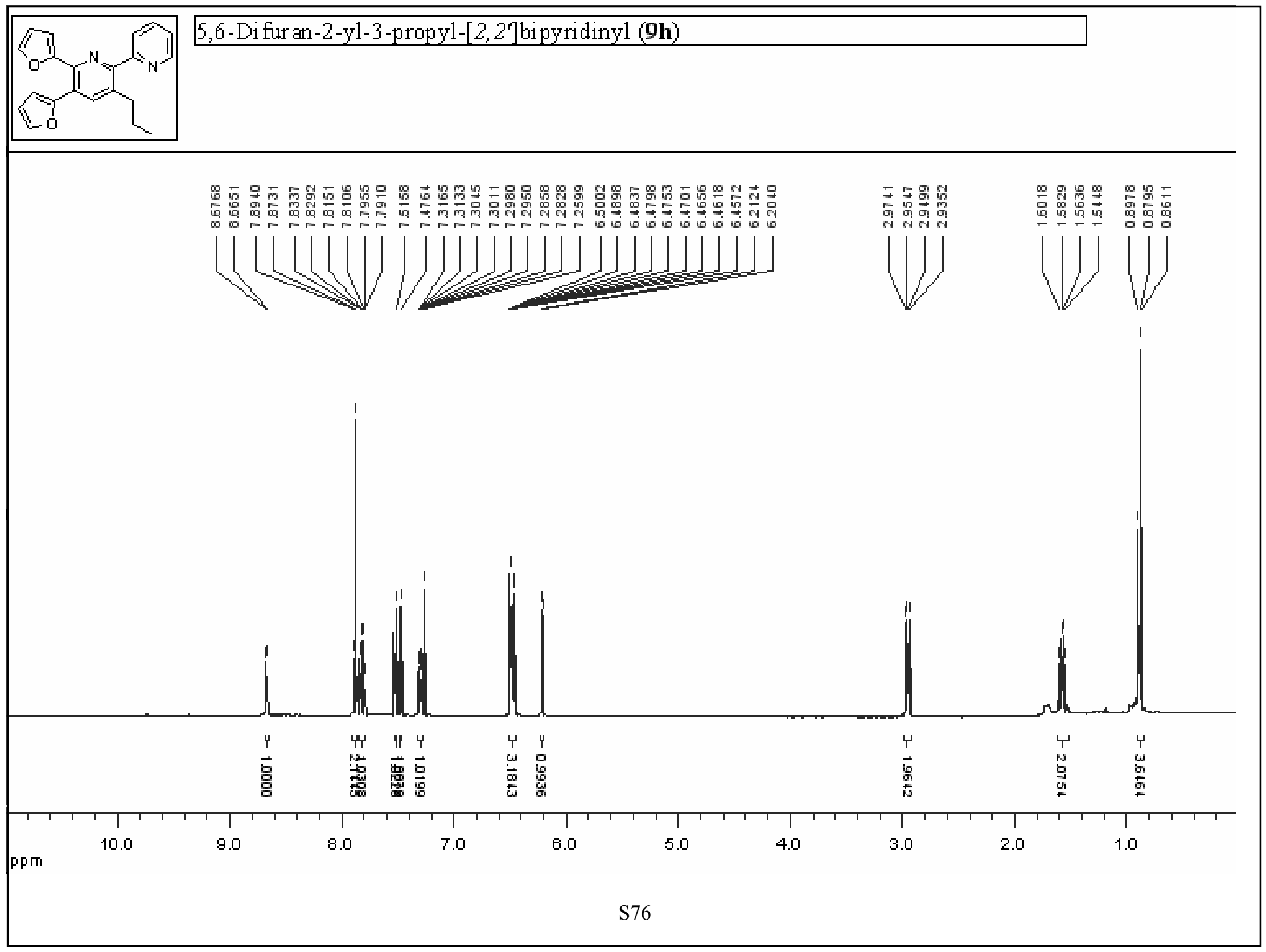




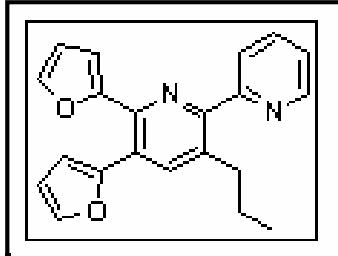

5,6-Di fur an-2-yl-3-propyl-[2,2] bipyridinyl (9h)

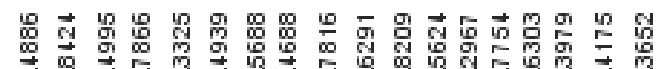

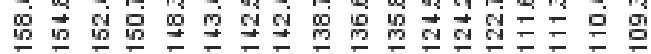
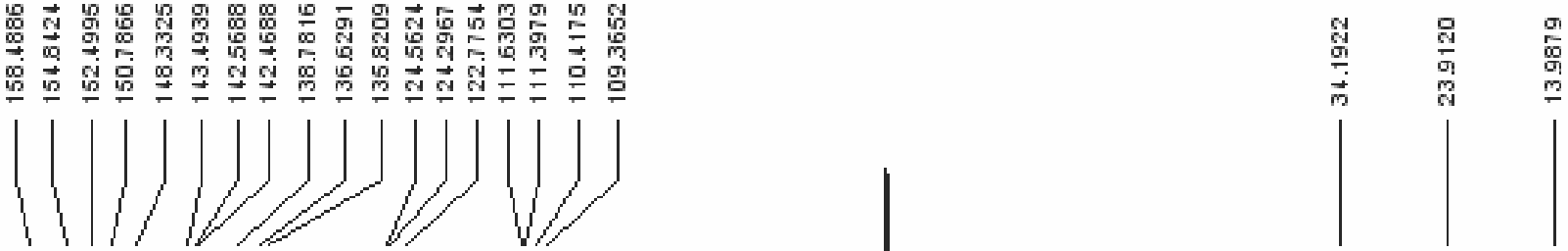

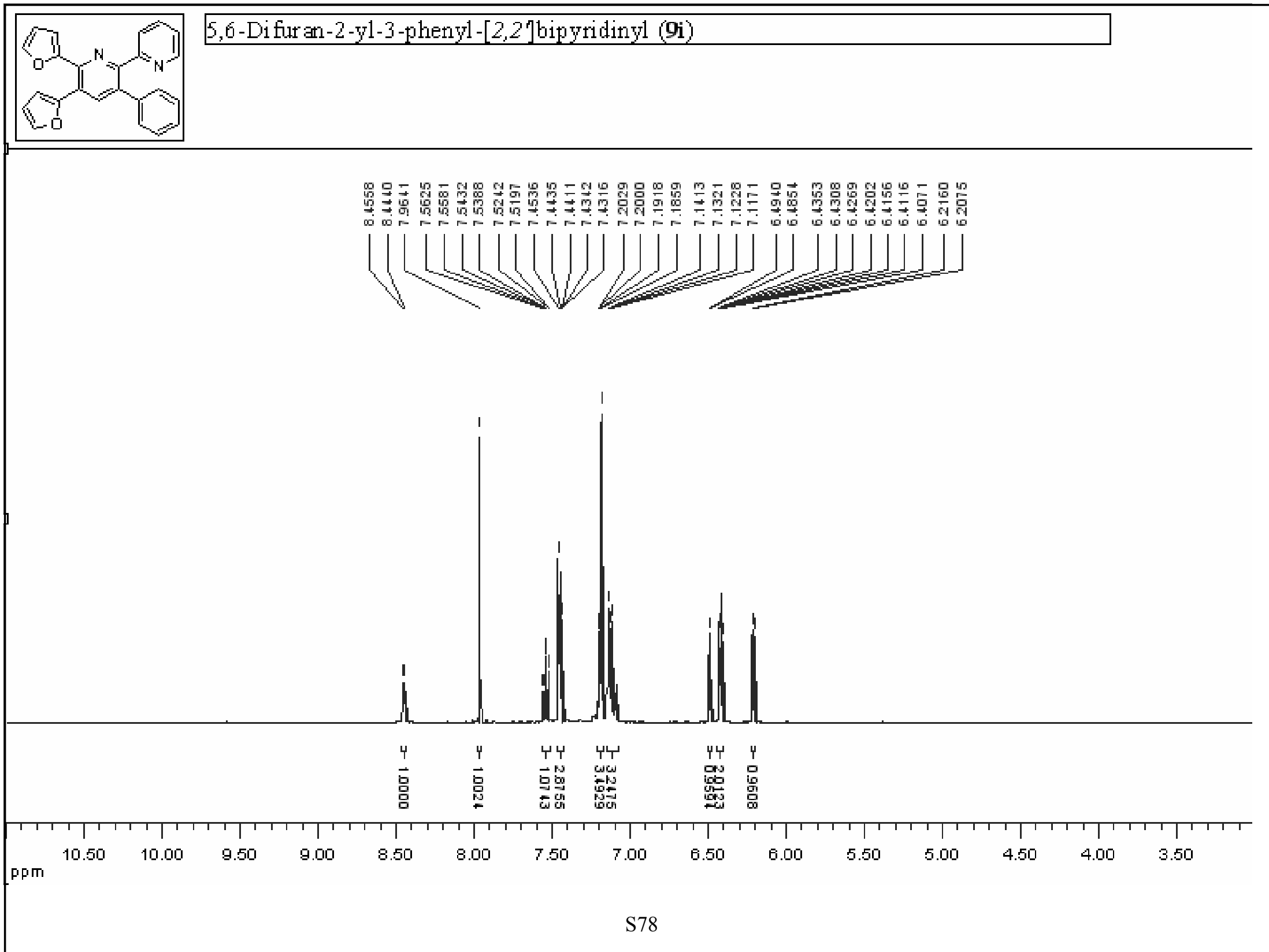


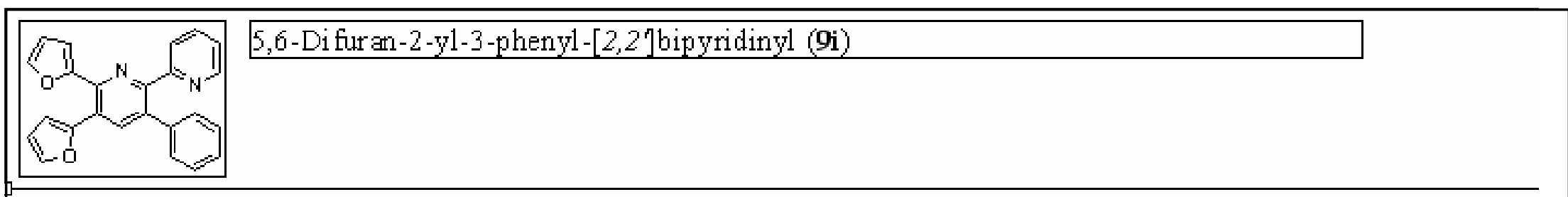

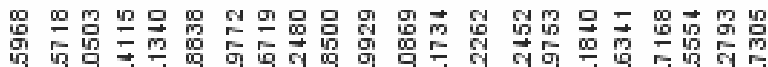

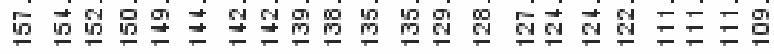
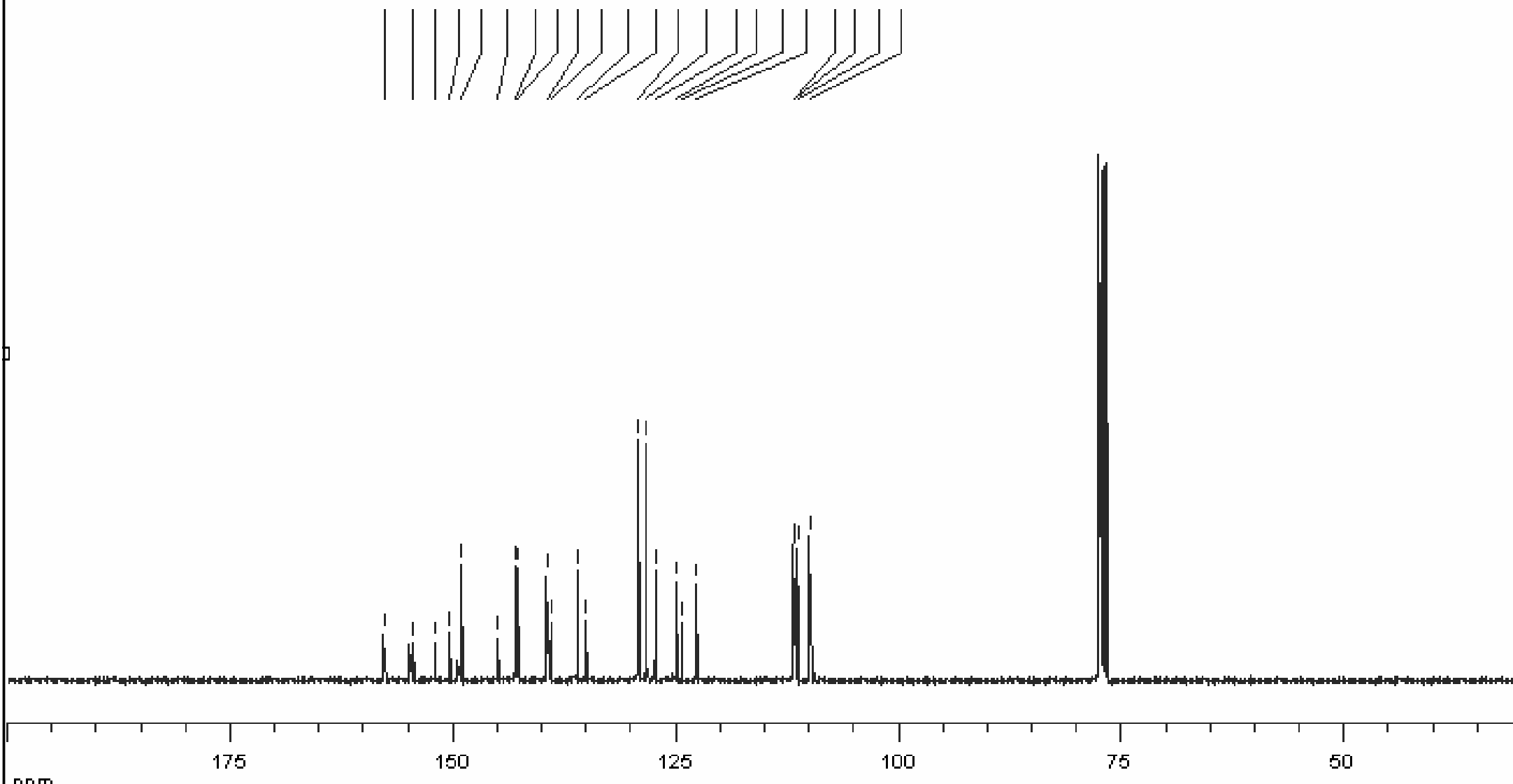


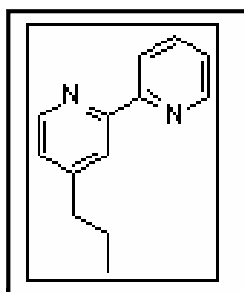

4-Fropyl-[2,2]bipyridinyl (9j)
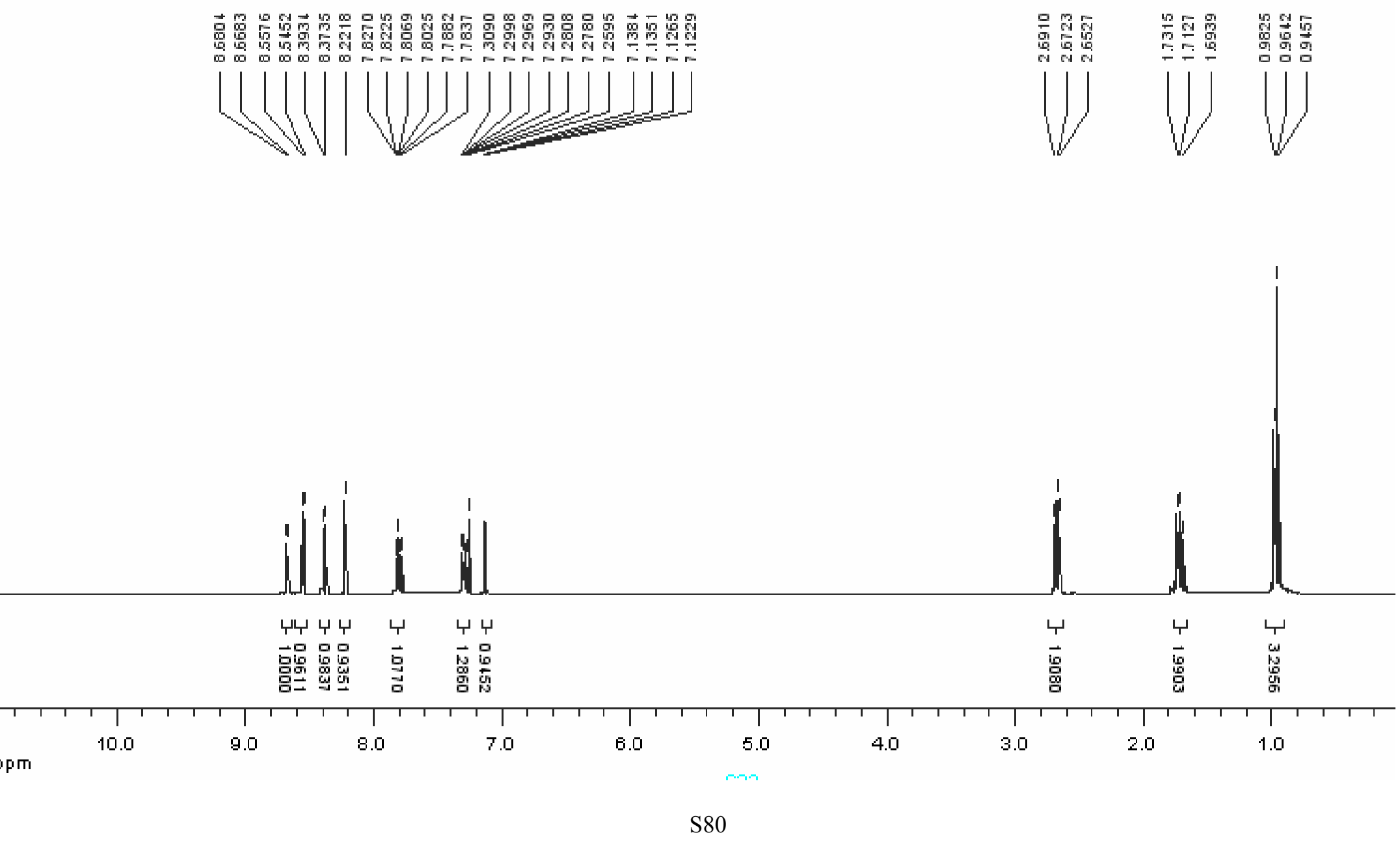


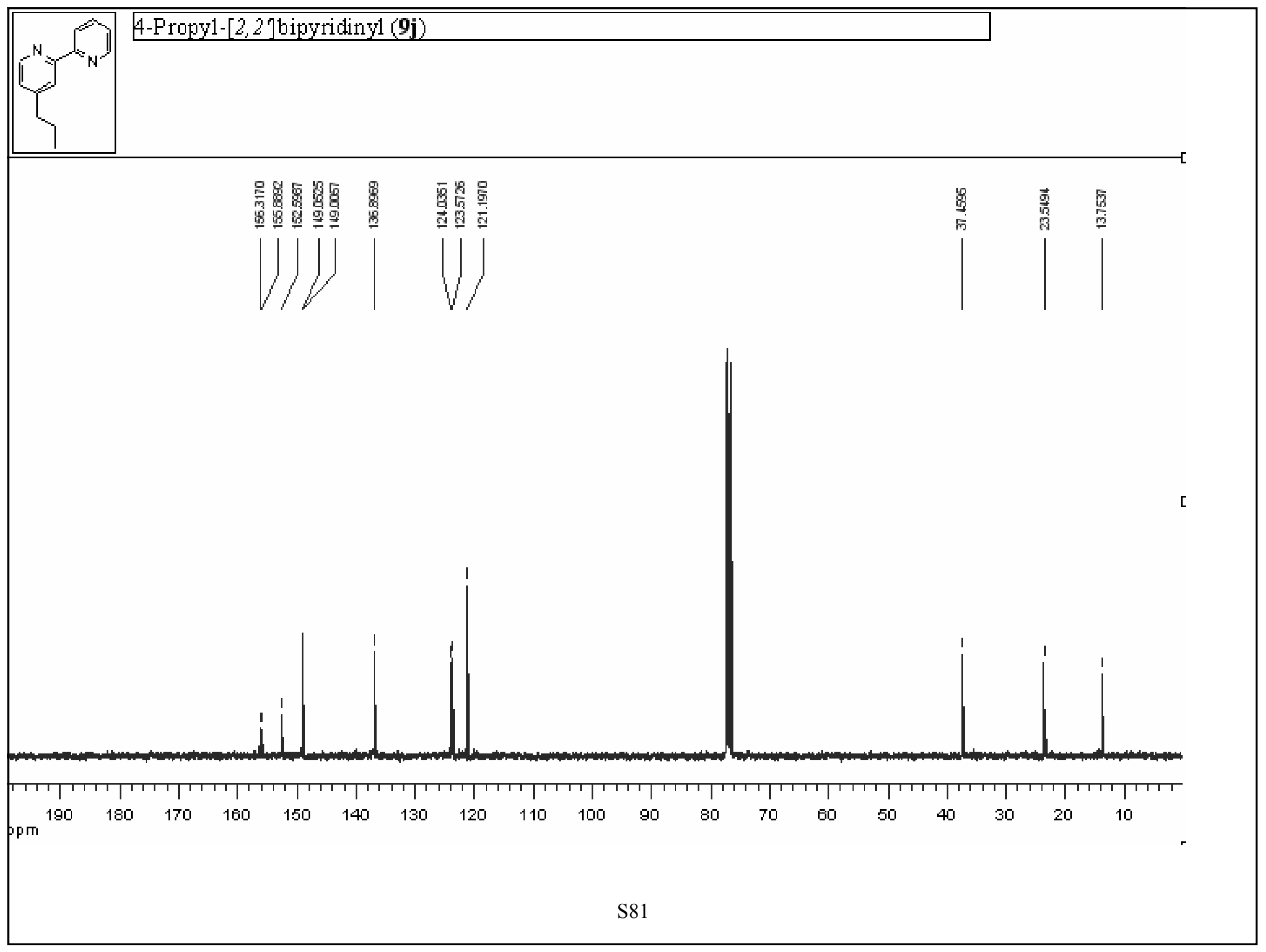

